\title{
First multispacecraft ion measurements in and near the Earth's magnetosphere with the identical Cluster ion spectrometry (CIS) experiment
}

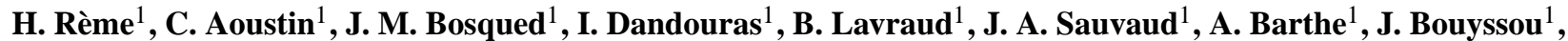
Th. Camus ${ }^{1}$, O. Coeur-Joly ${ }^{1}$, A. Cros $^{1}$, J. Cuvilo ${ }^{1}$, F. Ducay ${ }^{1}$, Y. Garbarowitz ${ }^{1}$, J. L. Medale ${ }^{1}$, E. Penou ${ }^{1}$, H. Perrier ${ }^{1}$, D. Romefort ${ }^{1}$, J. Rouzaud ${ }^{1}$, C. Vallat ${ }^{1}$, D. Alcaydé ${ }^{1}$, C. Jacquey ${ }^{1}$, C. Mazelle ${ }^{1}$, C. d'Uston ${ }^{1}$, E. Möbius ${ }^{2}$, L. M. Kistler ${ }^{2}$, K. Crocker ${ }^{2}$, M. Granoff ${ }^{2}$, C. Mouikis ${ }^{2}$, M. Popecki ${ }^{2}$, M. Vosbury ${ }^{2}$, B. Klecker ${ }^{3}$, D. Hovestadt $^{3}$, H. Kucharek ${ }^{3}$, E. Kuenneth $^{3}$, G. Paschmann ${ }^{3}$, M. Scholer ${ }^{3}$, N. Sckopke $(\dagger)^{3}$, E. Seidenschwang ${ }^{3}$, C. W. Carlson ${ }^{4}$, D. W. Curtis ${ }^{4}$, C. Ingraham ${ }^{4}$, R. P. Lin ${ }^{4}$, J. P. McFadden ${ }^{4}$, G. K. Parks ${ }^{4}$, T. Phan ${ }^{4}$, V. Formisano ${ }^{5}$, E. Amata ${ }^{5}$,

M. B. Bavassano-Cattaneo ${ }^{5}$, P. Baldetti ${ }^{5}$, R. Bruno ${ }^{5}$, G. Chionchio ${ }^{5}$, A. Di Lellis ${ }^{5}$, M. F. Marcucci ${ }^{5}$, G. Pallocchia ${ }^{5}$,

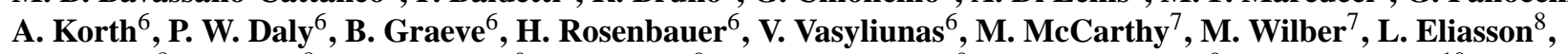
R. Lundin ${ }^{8}$, S. Olsen ${ }^{8}$, E. G. Shelley ${ }^{9}$, S. Fuselier ${ }^{9}$, A. G. Ghielmetti ${ }^{9}$, W. Lennartsson ${ }^{9}$, C. P. Escoubet ${ }^{10}$, H. Balsiger $^{11}$, R. Friedel ${ }^{12}$, J-B. Cao ${ }^{13}$, R. A. Kovrazhkin ${ }^{14}$, I. Papamastorakis ${ }^{15}$, R. Pellat ${ }^{16}$, J. Scudder ${ }^{17}$, and B. Sonnerup ${ }^{18}$

${ }^{1}$ CESR, BP 4346, 31028 Toulouse Cedex 4, France

${ }^{2} \mathrm{UNH}$, Durham, USA

${ }^{3}$ MPE, Garching, Germany

${ }^{4}$ SSL, Berkeley, USA

${ }^{5}$ IFSI, Roma, Italy

${ }^{6}$ MPAE, Lindau, Germany

${ }^{7}$ U. W., Seattle, USA

${ }^{8}$ IRF, Kiruna, Sweden

${ }^{9}$ Lockheed, Palo Alto, USA

${ }^{10}$ ESA/ESTEC, Noordwijk, the Netherlands

${ }^{11}$ Bern University, Bern, Switzerland

${ }^{12}$ Los Alamos National Laboratory NM, USA

${ }^{13}$ CCSAR, Beijing, China

${ }^{14}$ IKI, Moscow, Russia

${ }^{15}$ University of Crete, Greece

${ }^{16}$ Commissariat à l'Energie Atomique, Paris, France

${ }^{17}$ University of Iowa, USA

${ }^{18}$ Dartmouth College, NH, USA

Received: 13 April 2001 - Revised: 13 July 2001 - Accepted: 16 July 2001

\begin{abstract}
On board the four Cluster spacecraft, the Cluster Ion Spectrometry (CIS) experiment measures the full, threedimensional ion distribution of the major magnetospheric ions $\left(\mathrm{H}^{+}, \mathrm{He}^{+}, \mathrm{He}^{++}\right.$, and $\left.\mathrm{O}^{+}\right)$from the thermal energies to about $40 \mathrm{keV} / \mathrm{e}$. The experiment consists of two different instruments: a COmposition and DIstribution Function analyser (CIS1/CODIF), giving the mass per charge composition with medium $\left(22.5^{\circ}\right)$ angular resolution, and a Hot Ion Anal-
\end{abstract}

Correspondence to: H. Rème (Henri.Reme@cesr.fr) yser (CIS2/HIA), which does not offer mass resolution but has a better angular resolution $\left(5.6^{\circ}\right)$ that is adequate for ion beam and solar wind measurements. Each analyser has two different sensitivities in order to increase the dynamic range. First tests of the intruments (commissioning activities) were achieved from early September 2000 to mid January 2001, and the operation phase began on 1 February 2001. In this paper, first results of the CIS instruments are presented showing the high level performances and capabilities of the instru- 
ments. Good examples of data were obtained in the central plasma sheet, magnetopause crossings, magnetosheath, solar wind and cusp measurements. Observations in the auroral regions could also be obtained with the Cluster spacecraft at radial distances of 4-6 Earth radii. These results show the tremendous interest of multispacecraft measurements with identical instruments and open a new area in magnetospheric and solar wind-magnetosphere interaction physics.

Key words. Magnetospheric physics (magnetopause, cusp and boundary layers; magnetopheric configuration and dynamics; solar wind - magnetosphere interactions)

\section{Introduction}

The CIS instrument on-board the Cluster mission has been described in detail in Rème et al. (1997). This paper included a complete description of the instruments built for the Cluster-1 mission. However, after the dramatic crash of the Ariane 5 launch on 4 June 1996 at Kourou, four new CIS instruments were rebuilt for the Cluster- 2 mission. There are significant differences between the hardware, the software and the telemetry products for the CIS instruments from Cluster-1 to Cluster-2. For this reason, a good, up-to-date description of the instruments is given in this paper before the presentation of some first results. This paper must be the reference for the CIS Cluster- 2 instruments.

Note that different naming for the spacecraft numbers, the spacecraft names, the spacecraft flight model numbers and the CIS experiment flight model numbers have been used. Table 1 clarifies these different names and numbers.

\section{Scientific objectives and experiment capabilities}

The prime scientific objective of the CIS experiment is the study of the dynamics of magnetized plasma structures in and around the vicinity of the Earth's magnetosphere, with the determination, as accurately as possible, of the local orientation and the state of motion of the plasma structures required for macrophysics and microphysics studies. The four Cluster spacecraft, with relative separation distances that can be adjusted to spatial scales of the structures (a few hundred kilometers to several thousand kilometers), give for the first time the unambiguous possibility to distinguish spatial from temporal variations.

The CIS experiment has been designed to provide very substantial contributions to:

- the study of the solar wind/magnetosphere interaction;

- the dynamics of the magnetosphere, including storms, substorms, and aurora;

- the physics of the magnetopause and of the bow shock;

- the polar cusps and the plasma sheet boundary layer dynamics;

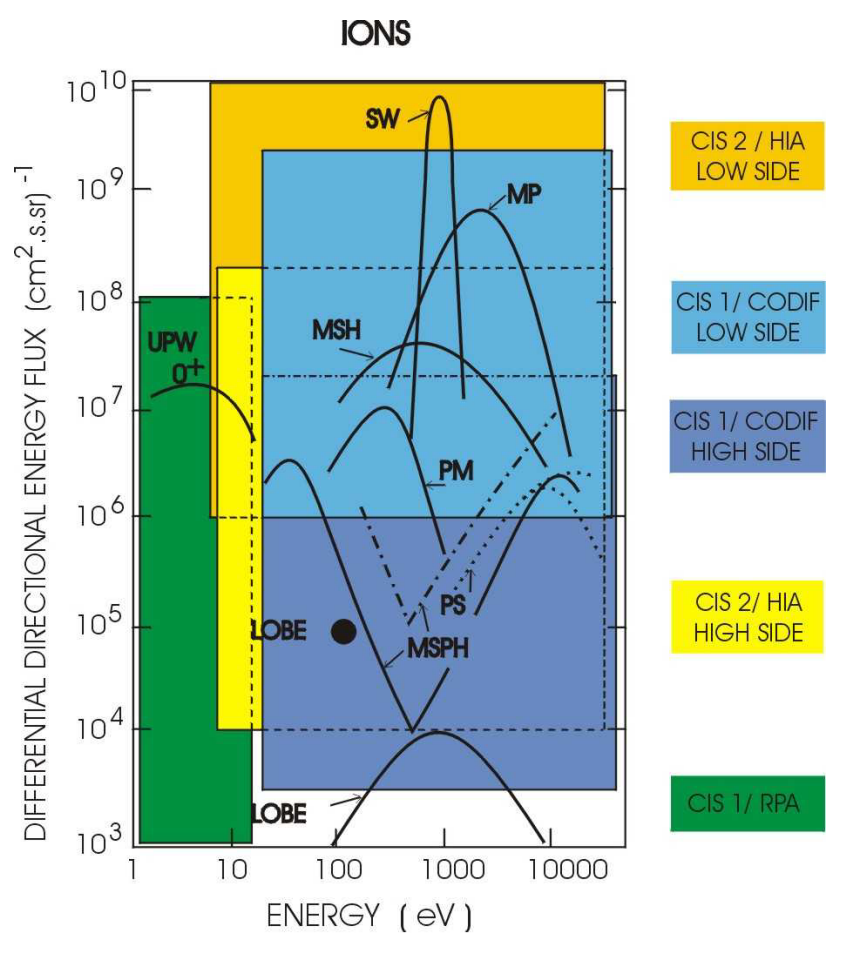

Fig. 1. Representative ion fluxes encountered along the Cluster orbit in the solar wind (SW), the magnetopause (MP), the magnetosheath (MSH), the plasma mantle (PM), the magnetosphere (MSPH), the plasma sheet (PS), the lobe and upwelling ions (UPW). The range of the different sensitivities of CIS1/CODIF (Low Side, High Side and RPA) and CIS2/HIA (Low $g$ and High $G$ ) are shown with different colours.

- the upstream foreshock and solar wind dynamics;

- the magnetic reconnection and the field-aligned current phenomena;

- the study of low energy ionospheric population.

The four Cluster spacecraft encounter ionic plasma with vastly diverse characteristics over the course of one year (Fig. 1). In order to study all of the plasma regions with the fluxes shown in Fig. 1, the CIS experiment needs, therefore, to be a highly versatile and reliable ionic plasma experiment, with the following requirements:

- A very great dynamic range is necessary in order to detect fluxes as low as those of the lobes, but also fluxes as high as solar wind fluxes, throughout the solar cycle.

- A broad energy range and a full $4 \pi$ angular coverage are necessary to provide a satisfactory and uniform coverage of the phase space with sufficient resolution. The angular resolution must be sufficient to be able to separate multiple populations, such as gyrating or transmitted ions from the main population downstream of the bow shock, and be able to detect fine structures in the distributions. 
Table 1. CIS Flight Model (FM) Naming

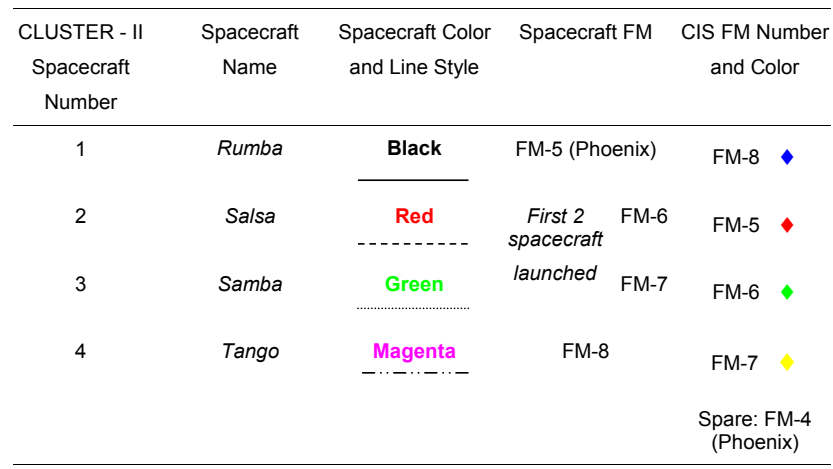

- A high angular and energy resolution in a limited energy and angular range for the detection of cold beams, such as the solar wind, is required. Due to the limited energy range required, a beam tracking algorithm has been implemented in order to follow the beam in velocity space. Moreover, in the foreshock regions, for example, any study of backstreaming ions requires the simultaneous observation of the solar wind cold beam and of the backstreaming particles. Therefore, in conjunction with the solar wind coverage described above, a coverage of the entire phase space including the sunward sector with a broad energy range is also used.

- In the case of sharp boundaries, such as discontinuities, it is necessary not to miss any information at the discontinuity; thus, a very efficient means of mode change, which allows adaptation to the local plasma conditions, is provided.

- Moments of the three-dimensional (3D) distribution (and of the sunward sector, in solar wind mode) are computed on board, with high time resolution to continuously generate key parameters that are necessary for event identification.

- In order to study detailed phenomena of complex magnetospheric plasma physics, multiple particle populations must be identified and characterized; therefore, a 3D distribution is needed. In order to transmit the full 3D distribution while overcoming the telemetry rate limitations, a compression algorithm has been introduced, which allows for an increased amount of information to be transmitted.

To achieve the scientific objectives, the CIS instrumentation has been designed to simultaneously satisfy the following criteria on the four spacecraft:

- Provide uniform coverage of ions over the entire $4 \pi$ steradian solid angle with good angular resolution.

- Separate the major mass ion species from the solar wind and ionosphere, i.e. those which contribute significantly

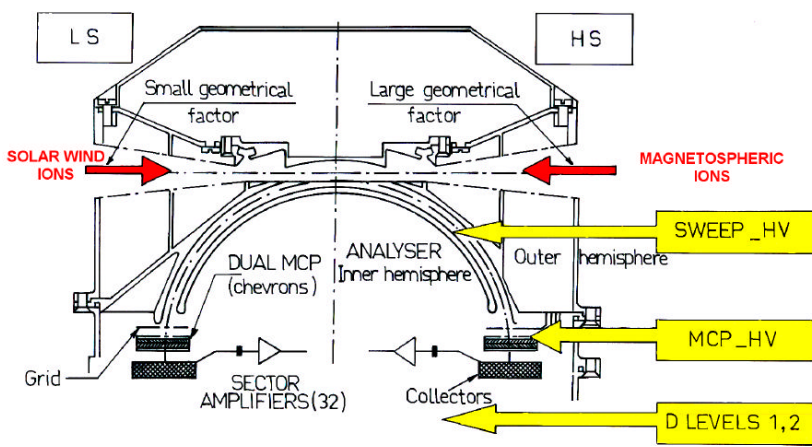

Fig. 2. Cross sectional view of the HIA analyser.

to the total mass density of the plasma (generally, $\mathrm{H}^{+}$, $\mathrm{He}^{++}, \mathrm{He}^{+}$, and $0^{+}$).

- Have high sensitivity and large dynamic range $\left(\geq 10^{7}\right)$ to support high time resolution measurements over the wide range of plasma conditions to be encountered in the Cluster mission (Fig. 1).

- Have high $\left(5.6^{\circ} \times 5.6^{\circ}\right)$ and flexible angular sampling resolution to support measurements of ion beams and the solar wind.

- Have the ability to routinely generate on board the fundamental plasma parameters for major ion species, with one spacecraft spin time resolution $(4 \mathrm{~s})$. These parameters include the density $(n)$, velocity vector $(\boldsymbol{V})$, pressure tensor $(\boldsymbol{P})$, and heat flux vector $(\boldsymbol{H})$.

- Cover a wide range of energies, from spacecraft potential to about $40 \mathrm{keV} / \mathrm{e}$.

- Have versatile and easily programmable operating modes and data processing routines to optimize the data collection for specific scientific studies and widely varying plasma regimes.

To satisfy all these criteria, the CIS package consists of two different instruments: a Hot Ion Analyser (HIA) sensor and a time-of-flight ion COmposition and DIstribution Function (CODIF) sensor. The CIS plasma package is versatile and is capable of measuring both the cold and hot ions of Maxwellian and non-Maxwellian populations (for example, beams) from the solar wind, the magnetosheath, and the magnetosphere (including the ionosphere) with sufficient angular, energy and mass resolutions to accomplish the scientific objectives. The time resolution of the instrument is sufficiently high to follow density or flux oscillations at the gyrofrequency of $\mathrm{H}^{+}$ions in a magnetic field of $10 \mathrm{nT}$ or less. Such field strengths can be frequently encountered by the Cluster mission. Oscillations of $\mathrm{O}^{+}$at the gyrofrequency can be resolved outside 6-7 $R_{E}$. Hence, this instrument package provides the ionic plasma data required to meet the Cluster science objectives (Escoubet and Schmidt, 1997). 


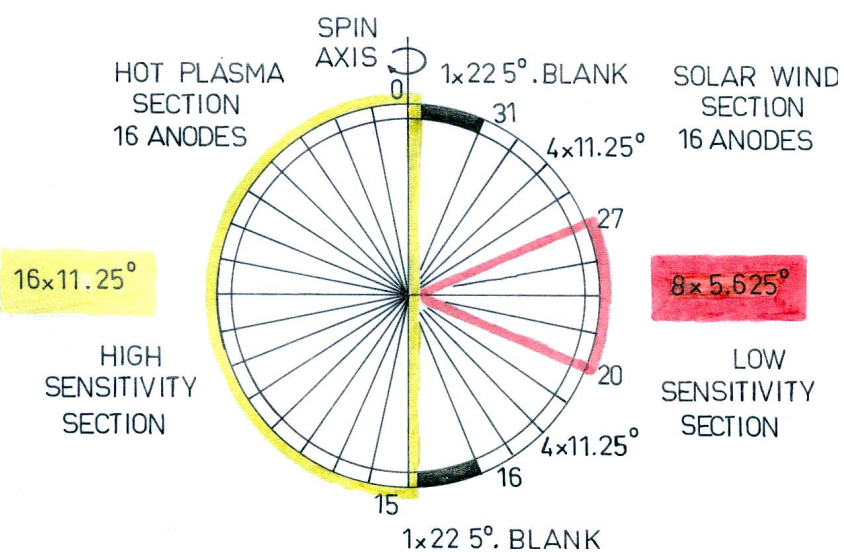

Fig. 3. Principle of the HIA anode sectoring.

\section{The Hot Ion Analyser (HIA)}

The Hot Ion Analyser (HIA) instrument combines the selection of incoming ions according to the ion energy per charge by electrostatic deflection in a symmetrical, quadrispherical analyser which has a uniform angle-energy response with a fast imaging particle detection system. This particle imaging is based on microchannel plate (MCP) electron multipliers and position encoding discrete anodes.

\subsection{Electrostatic analyser description}

Basically, the analyser design is a symmetrical, quadrispherical electrostatic analyser which has a uniform $360^{\circ}$ discshaped field of view (FOV) and an extremely narrow angular resolution capability. This symmetric quadrisphere or "top hat" geometry (Carlson et al., 1982) has been successfully used on numerous sounding rocket flights, as well as on the AMPTE/IRM, Giotto and WIND spacecraft (Paschmann et al., 1985; Rème et al., 1987; Lin et al., 1995).

The symmetric quadrisphere consists of three concentric spherical elements. These three elements are an inner hemisphere, an outer hemisphere which contains a circular opening, and a small circular top cap which defines the entrance aperture. This analyser is classified as quadrispherical simply because the particles are deflected through $90^{\circ}$. In the analyser, a potential is applied between the inner and outer plates and only charged particles with a limited range of energy and an initial azimuth angle are transmitted. The particle exit position is a measure of the incident polar angle which can be resolved by a suitable position-sensitive detector system. The symmetric quadrisphere makes the entire analyser, including the entrance aperture, rotationally symmetric. The focusing characteristics are independent of the polar angle. We use the following convention: the angle about the spin axis is the azimuth angle, whereas the angle out of the spin plane is called the polar angle.

The symmetrical quadrispherical analyser has good focusing properties, sufficient energy resolution, and the large ge-
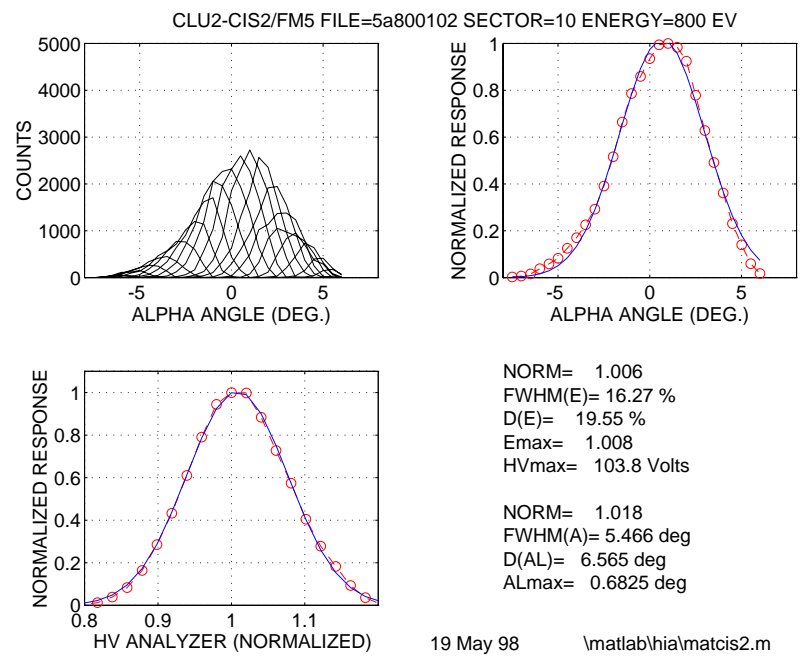

Fig. 4. Typical energy (top curves) and angular (bottom curve) resolutions of the HIA analyser (flight model 5), for an energy beam of $800 \mathrm{eV}$; the energy resolution is about $19.6 \%$ and the intrinsic azimuthal resolution is about $6.6^{\circ}$.

ometrical factor of a quadrisphere. Due to symmetry, it does not have the deficiencies of the conventional quadrisphere, namely the limited polar angle range and the severely distorted response characteristics at large polar angles, and it has an uniform polar response.

The HIA instrument has $2 \times 180^{\circ}$ FOV sections parallel to the spin axis, with two different sensitivities and a ratio of about 25 (depending of the flight model and precisely known calibrations), corresponding, respectively, to the "high $G$ " and "low $g$ " sections. The "low $g$ " section allows for the detection of the solar wind and the required high angular resolution is achieved through the use of $8 \times 5.625^{\circ}$ central anodes, with the remaining 8 sectors having, in principle, a $11.25^{\circ}$ resolution; the $180^{\circ}$ "high $G$ " section is divided into 16 anodes, $11.25^{\circ}$ each. In reality, sectoring angles are, respectively, $\sim 5.1^{\circ}$ and $\sim 9.7^{\circ}$, as demonstrated by calibrations (see Sect. 3.5). This configuration provides "instantaneous", 2D distributions sampled once per $62.5 \mathrm{~ms}(1 / 64$ of one spin, i.e. $5.625^{\circ}$ in azimuth), which is the nominal sweep rate of the high voltage applied to the inner plate of the electrostatic analyser to select the energy of the transmitted particles. For each sensitivity section, a full $4 \pi$ steradian scan is completed every spin of the spacecraft, i.e. $4 \mathrm{~s}$, giving a full, 3D distribution of the ions in the energy range of $5 \mathrm{eV} \mathrm{e}^{-1}$ to $32 \mathrm{keV} \mathrm{e}^{-1}$ (the analyser constant being $\sim 6.70$ ). Figure 2 provides a cross sectional view of the HIA electrostatic analyser. The inner and outer plate radii are $37.75 \mathrm{~mm}$ and $40.20 \mathrm{~mm}$, respectively. The analyser has an entrance aperture which collimates the field of view, defines the two geometrical factors and blocks the solar UV radiation.

\subsection{Detection system}

A pair of half-ring microchannel plates (MCP) in a chevron pair configuration detects the particles at the exit of the elec- 
Table 2. Main features and measured parameters of the CIS experiment

- Full 3D ion distribution functions

- Flux as a function of time, mass and pitch-angle

- Moments of the distribution functions : density, bulk velocity, pressure tensor, heat flux vector

- Beams

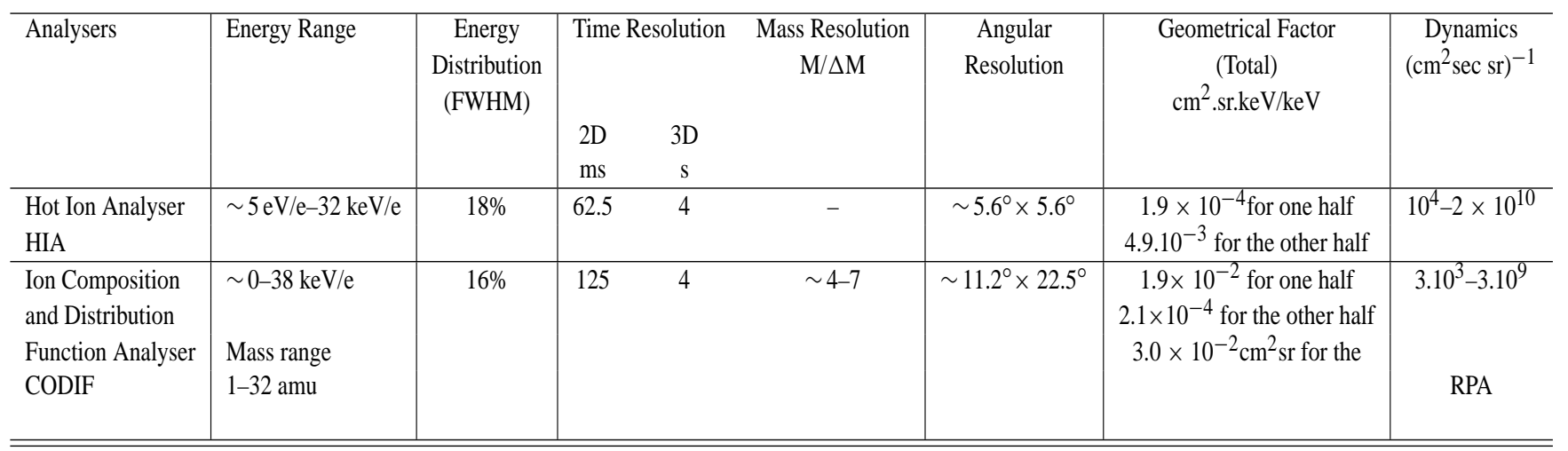

\begin{tabular}{l|l|l|l}
\hline Analysers & Full Instantaneous Field of View & Mass & $\begin{array}{l}\text { Power } \\
\text { (Nominal Operations) }\end{array}$ \\
\hline Hot Ion Analyser HIA & $8^{\circ} \times 360^{\circ}$ & $2.45 \mathrm{~kg}$ & 2.82 watts \\
Ion Composition & $8^{\circ} \times 360^{\circ}$ & $8.39 \mathrm{~kg}$ & 6.96 watts \\
Function Analyser CODIF & & & \\
\hline \hline
\end{tabular}

CIS total raw CIS Total Weight: $10.84 \mathrm{~kg}$ without harness

Average power: 9.78 watts

CIS Telemetry: $\sim 5.5 \mathrm{kbit} / \mathrm{s}$

Expected total bit number (for the four spacecraft): $10^{12}$ bits

trostatic analyser. The plates form a $2 \times 180^{\circ}$ ring shape, each $1 \mathrm{~mm}$ thick with an inter-gap of $\sim 0.02 \mathrm{~mm}$, an inner diameter of $75 \mathrm{~mm}$ and an outer diameter of $85 \mathrm{~mm}$. The MCPs have $12.5 \mu \mathrm{m}$ straight microchannels, with a bias angle of $8^{\circ}$ to reduce variations in MCP efficiency with azimuthal direction. The chevron configuration, with double thickness plates, provides a saturated gain of $2 \times 10^{6}$, with a narrow pulse height distribution. The plates have a high strip current to provide a fast counting capability. For better detection, efficiency ions are post-accelerated by a $\sim 2300 \mathrm{~V}$ potential applied between the front of the first MCP and a high-transparency grid located $\sim 1 \mathrm{~mm}$ above. The anode collector behind the MCPs is divided into 32 sectors, each connected to its own pulse amplifier (Fig. 3). The main performances of the HIA sensor are summarised in Table 2.

\subsection{Sensor electronics}

Signals from each of the $32 \mathrm{MCP}$ sectors are sent through 32 specially designed, very fast A121 charge-sensitive amplifier/discriminators that are able to count at rates as high as
$5 \mathrm{MHz}$. Output counts from the 32 sectors are accumulated in 48 counters (including 16 redundant counters for the solar wind), thus providing the basic angular resolution matrix according to the resolution of the anode sectoring.

According to the operational mode, several angular resolutions can be achieved:

- In the normal resolution mode, the full 3D distributions are covered in $\sim 11.25^{\circ}$ angular bins ("high $G$ " geometrical factor); this is the basic mode inside the magnetosphere;

- In the high resolution mode the best angular resolution, $\sim 5.6^{\circ} \times 5.6^{\circ}$, is achieved within a $45^{\circ}$ sector centred on the Sun direction, using the "low $g$ " geometrical factor section; this mode is dedicated to the detection of the solar wind and near-ecliptic narrow beams.

\subsubsection{High voltage power supplies}

HIA needs a high-voltage power supply to polarise MCPs at $\sim 2300-2500 \mathrm{~V}$ and a sweeping high voltage applied on the 
inner plate of the electrostatic analyser. The high voltages to polarise the MCPs are adjustable under the control of the data processor system (DPS) microprocessor.

The energy/charge of the transmitted ions is selected by varying the deflection voltage applied to the inner plate of the electrostatic analyser, between 4800 and $0.7 \mathrm{~V}$. The exponential sweep variation of the deflection voltage is synchronised with the spacecraft spin period. The sweep should consist of many small steps that give effectively a continuous sweep. The counter accumulation time defines the number of energy steps, i.e. 31 or 62 count intervals per sweep. The covered energy range and the sweeping time are controlled by the onboard processor through a 12-bit DAC and a division in the two ranges for the sweeping high voltage. Therefore, the number of sweeps per spin, the amplitude of each sweep and the sweeping energy range can be adjusted according to the mode of operation (solar wind tracking, beam tracking, etc.). In the basic and nominal modes, the sweep of the total energy range is repeated 64 times per spin, i.e. once every $62.5 \mathrm{~ms}$, giving a $\sim 5.6^{\circ}$ resolution in azimuth resolution. In the solar wind mode, HIA sweep is truncated when "high $G$ " is facing the Sun in order to avoid the solar wind detection with "high $G$ " and to protect the MCP lifetime.

\subsection{In-flight calibration test}

A pulse generator can stimulate the 32 amplifiers that are under the processor control. In this way, important functions of the HIA instrument and of the associated on board processing can easily be tested. A special test mode is implemented for health checking of the microprocessor by making ROM check sums and RAM tests. The sweeping high voltage can be tested by measuring the voltage value of each individual step, and the MCP gain can be checked by occasionally stepping MCP HV and by adjusting the discrimination level of the charge amplifiers. Performances of the HIA sensor are shown in Table 2 and in Fig. 1.

\subsection{HIA performances}

Pre-flight and extensive calibrations of all four HIA flight models and of the spare model were performed at the CESR vacuum test facilities in Toulouse, using large and stable ion beams of different ion species and variable energies, detailed studies of MCPs and gain level variations, MCP matching, and angular-energy resolution for each sector from a few tens of $\mathrm{eV}$ up to $30 \mathrm{keV}$. Typical performances of the HIA instrument are reproduced in Figs. 4, 5 and 6. Figure 4 shows an example of the typical energy and angular resolutions of the HIA analyser (flight model FM5/SC2) for an energy beam of $800 \mathrm{eV}$; in this case, the energy resolution is $16.3 \%$ and the intrinsic azimutal resolution $\sim 5.5^{\circ}$. On average the analyser energy resolution $\Delta E / E$ (FWHM) is $\sim 17 \%$, almost independent of anode sectors and energy; thus the intrinsic HIA velocity resolution is $\sim 9 \%$, only about half of the average solar wind spread value. This is equivalent to an angular resolution of $\sim 5^{\circ}$ and is thus, quite consistent with the an- gular resolution capabilities of the instrument, i.e. $\sim 5.9^{\circ}$ (FWHM) in the azimuthal angle, as indicated in Fig. 4, and $\sim 5.6^{\circ}$ in the polar angle. As seen in the example of Fig. 5 for the model FM6/SC3, the polar resolution stays, as expected, almost constant at $\sim 9.70^{\circ}$ over the 16 sectors (anodes 0 to 15) that constitute the "high $G$ " section (Fig. 5). Anodes 16 to 31 correspond to the "low $g$ " section and their response transmission is attenuated by a factor of about 25 (depending on the flight model, see Table 3 ) due to the presence of a pin-hole grid placed in front of the $180^{\circ}$ collimator; the polar resolution of sectors 20 to 27 is $\sim 5.2^{\circ}$. Figure 6 shows the excellent agreement for the transmission width for the four flight models and the spare model. Thus, when compared to the basic sectoring, $\sim 5.6^{\circ}$ and $\sim 11.2^{\circ}$, all effective polar resolutions are reduced due to the existence of an insulation space between the discrete anodes, as well as by the presence of support posts within the field of view. Finally, experimental energy, angle resolutions and transmission factors are introduced in the geometrical factor used to compute moments of the distribution function.

\subsubsection{UV Rejection}

A number of very interesting events are expected to occur when the HIA spectrometers face the Sun (2 times/spin): of course, the intense solar wind, but also, for example, tailward ion beams flowing along the Plasma Sheet Boundary Layer (PSBL). A number of measures were applied in order to suppress or limit the solar UV contamination. Part of the UV is rejected by the entrance collimator; moreover, the inner surface of the outer sphere is scalloped and both spheres (and all internal parts) are treated and coated with a special black cupric sulfide. Extensive vacuum chamber tests of the HIA analysers were performed, using a calibrated continuous discharge source for extreme UV at He-584 $\AA$ and L $\alpha 1215 \AA$ lines. Reduction of the solar UV light reflectance at the $\mathrm{L} \alpha$ line was demonstrated in Rème et al. (1997) for Cluster-1 flight models. The resulting maximum count rate recorded by the sunward looking sector $\left(11.2^{\circ}\right.$ wide $)$ for these models was about 80 counts s $^{-1}$ (for an intensity equivalent to 3 Sun intensity units), and the UV contamination was distributed over about $\sim 100^{\circ}$ in the polar angle; this UV contamination was judged acceptable. Figure 7a shows this UV contamination for a Cluster-1 HIA flight model. However, for Cluster-2 flight models, it was decided to improve the UV rejection by changing the scalloping of the outer sphere. The result was excellent. Figure $7 b$ shows the UV test result for the FM4 spare model under the same conditions as that of Fig. 7a for Cluster-1. The contamination is divided by a factor of about 700. In Fig. 8, an example of measurements by the HIA FM6/SC3 instrument in the central plasma sheet on 11 September 2000 is shown. "Natural counts" are detected between about $150 \mathrm{eV}$ and $14 \mathrm{keV}$. The UV rejection is excellent since there are no counts in the Sun direction ( + and $-180^{\circ}$ ) for the highest energy ion measurements where no natural particles are present in this region. In the same figure, the absence of counts at the lowest and highest energies, 


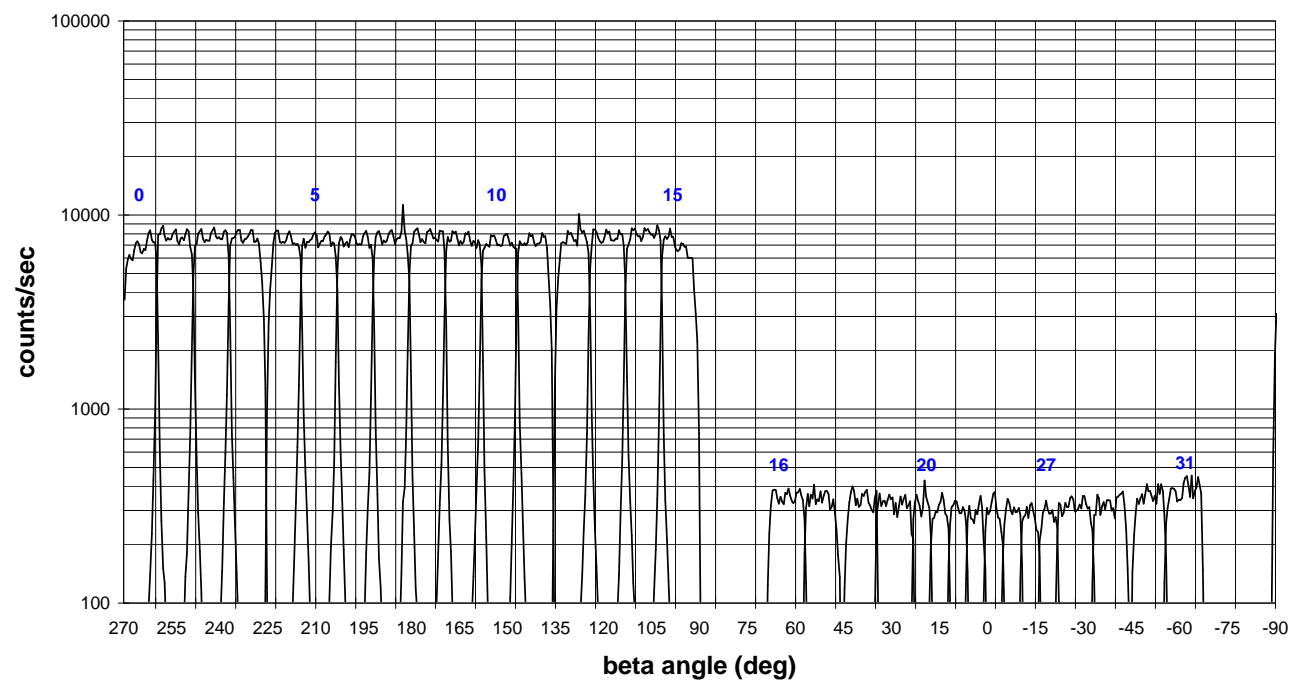

Fig. 5. Relative transmission of the $16 \mathrm{HIA}$ High $G$ (from $270^{\circ}$ to $90^{\circ}$ ) and 16 HIA Low $g$ (from $90^{\circ}$ to $-90^{\circ}$ ) polar sectors (see Fig. 3 ) for FM6 at $5 \mathrm{keV}$.

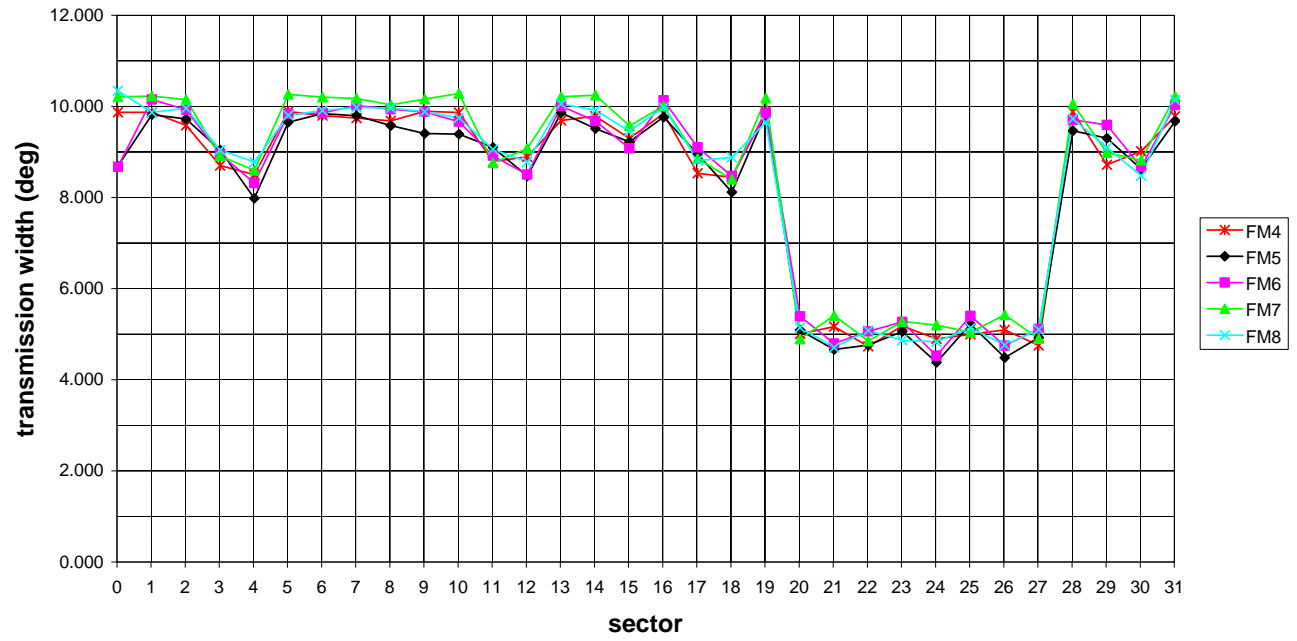

Fig. 6. Beta sector transmission of HIA for the four flight models and the spare model. Results are very similar for the five models. Sectors $0-15$ correspond to the High $G$ and 16-31 correspond to the low $g$. Transmission in sectors 20-27 is divided by two, as expected from the geometry.

i.e. below and above the central plasma sheet, particle energies show that the HIA sensors have a very low MCP and amplifier noise.

\section{The ion composition and distribution function anal- yser (CODIF)}

The CODIF instrument is a high-sensitivity, mass-resolving spectrometer with an instantaneous $360^{\circ} \times 8^{\circ}$ field of view to measure complete 3D distribution functions of the major ion species within one spin period of the spacecraft. Typically, these include $\mathrm{H}^{+}, \mathrm{He}^{++}, \mathrm{He}^{+}$and $\mathrm{O}^{+}$. The sensor primarily covers the energy range between 0.02 and $38 \mathrm{keV} / \mathrm{charge}$. With an additional Retarding Potential Analyser (RPA) device in the aperture system of the sensor with pre-acceleration for energies below $25 \mathrm{eV} / \mathrm{e}$, the range is extended to energies as low as the spacecraft potential. Hence, CODIF covers the core of all plasma distributions of importance to the Cluster mission.

To cover the large dynamic range required for accurate measurements in the low-density plasma of the magnetotail and the dense plasma in the magnetosheath/cusp/ boundary layer, it is mandatory that CODIF employ two different sensitivities. The minimum number of counts in a distribution needed for computing the basic plasma parameters, such as the density, is about 100 . These must be accumulated in 1 spin in order to provide the necessary time resolution. However, the maximum count rate which the time-of-flight system can handle is $\sim 10^{5}$ counts $\mathrm{s}^{-1}$ or $4 \times 10^{5}$ counts $\operatorname{spin}^{-1}$. This means that the dynamic range achievable with 
Table 3. Energy resolution, analyser constant and geometrical factor per anode for the four HIA flight models and for the spare model. The high geometrical factor corresponds to sectors $0-15$ and the low geometrical factor corresponds to sectors 20-27 (see Fig. 3). These parameters are slightly different from the parameters of the Cluster- 1 models due to the modification of the sphere scalloping design used to obtain a better UV rejection (see below)

\begin{tabular}{|c|c|c|c|c|c|c|c|c|c|}
\hline Parameter & \multicolumn{2}{|c|}{ FM5/SC2 } & \multicolumn{2}{|c|}{ FM6/SC3 } & \multicolumn{2}{|c|}{ FM7/SC4 } & \multicolumn{2}{|c|}{ FM8/SC1 } & FM4/SPARE \\
\hline $\begin{array}{l}\text { Geometrical } \\
\text { Factor }\end{array}$ & $G$ & $g$ & $G$ & $g$ & $G$ & $g$ & $G$ & $g$ & $\begin{array}{ll}G & g\end{array}$ \\
\hline $\begin{array}{c}\text { Attenuation } \\
\text { grid }\end{array}$ & 1 & $1 / 24$ & 1 & $1 / 22$ & 1 & $1 / 25$ & 1 & $1 / 25$ & 1 \\
\hline$\Delta E / E, \%$ & 16.44 & 15.94 & 16.66 & 15.96 & 17.61 & 17.23 & 16.59 & 16.07 & $\begin{array}{ll}17.19 & 17.32\end{array}$ \\
\hline $\begin{array}{l}K \text { analyser } \\
\text { (all sectors) }\end{array}$ & \multicolumn{2}{|c|}{7.629} & \multicolumn{2}{|c|}{7.341} & \multicolumn{2}{|c|}{7.042} & \multicolumn{2}{|c|}{7.685} & 7.454 \\
\hline $\begin{array}{c}\text { Geometrical } \\
\text { Factor per } \\
\text { anode } \\
\left(\mathrm{cm}^{2} . s r . k e V /\right. \\
\mathrm{keV})\end{array}$ & $\begin{array}{c}3.00 \\
\times 10^{-4}\end{array}$ & $\begin{array}{c}5.769 \\
\times 10^{-6}\end{array}$ & $\begin{array}{c}3.805 \\
\times 10^{-4}\end{array}$ & $\begin{array}{l}1.084 \\
\times 10^{-5}\end{array}$ & $\begin{array}{c}3.403 \\
\times 10^{-4}\end{array}$ & $\begin{array}{c}6.368 \\
\times 10^{-6}\end{array}$ & $\begin{array}{c}4.966 \\
\times 10^{-4}\end{array}$ & $\begin{array}{l}1.226 \\
\times 10^{-5}\end{array}$ & \\
\hline
\end{tabular}
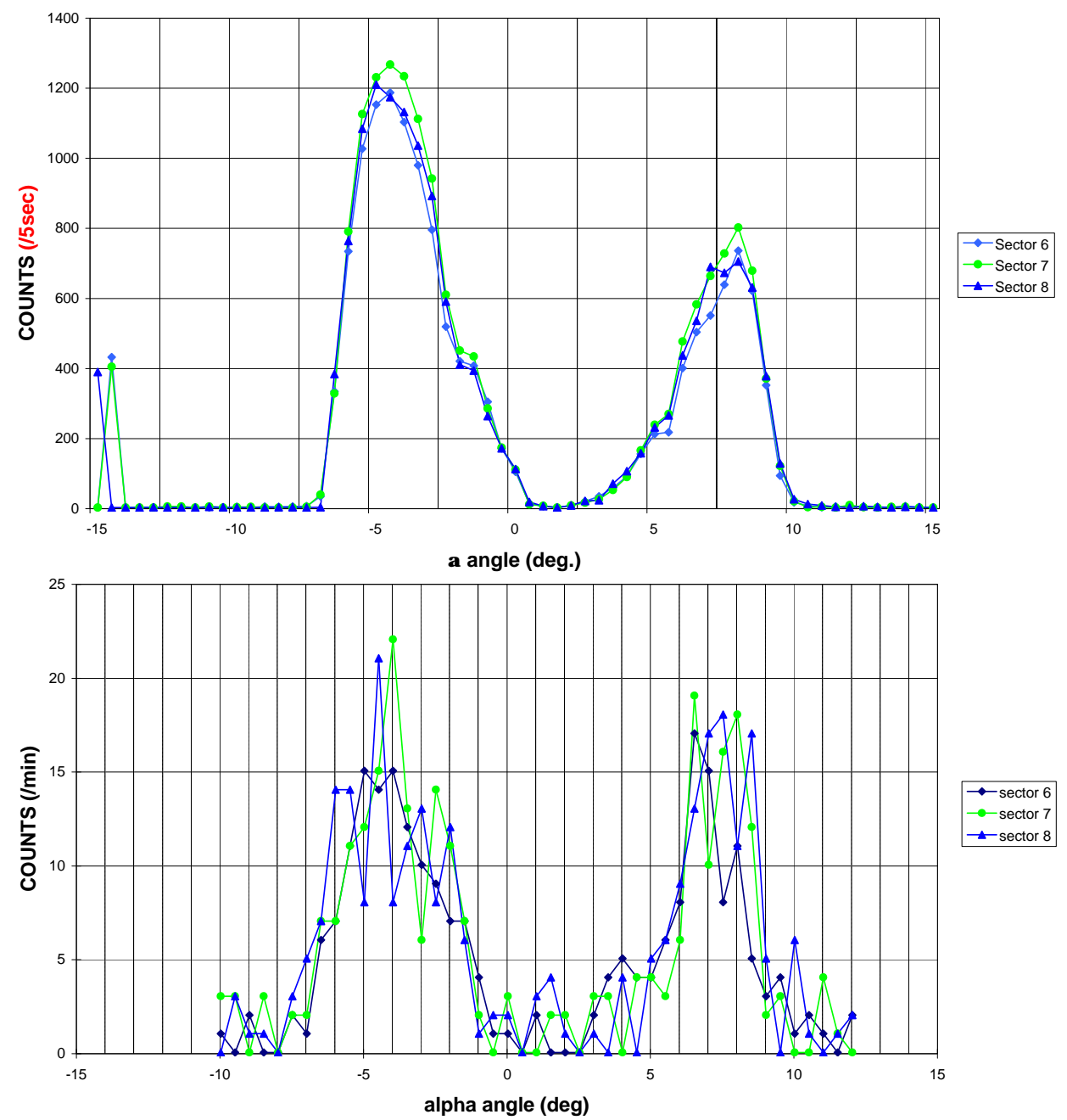

Fig. 7. UV effects on a Cluster-1 HIA model (counts/5s) in Fig. 7(a) and on a Cluster-2 HIA model (counts/min) in Fig. 7( b) in the function of the polar angle. The background for Cluster- 2 has been divided by two orders of magnitude by improving the analyser scalloping. 


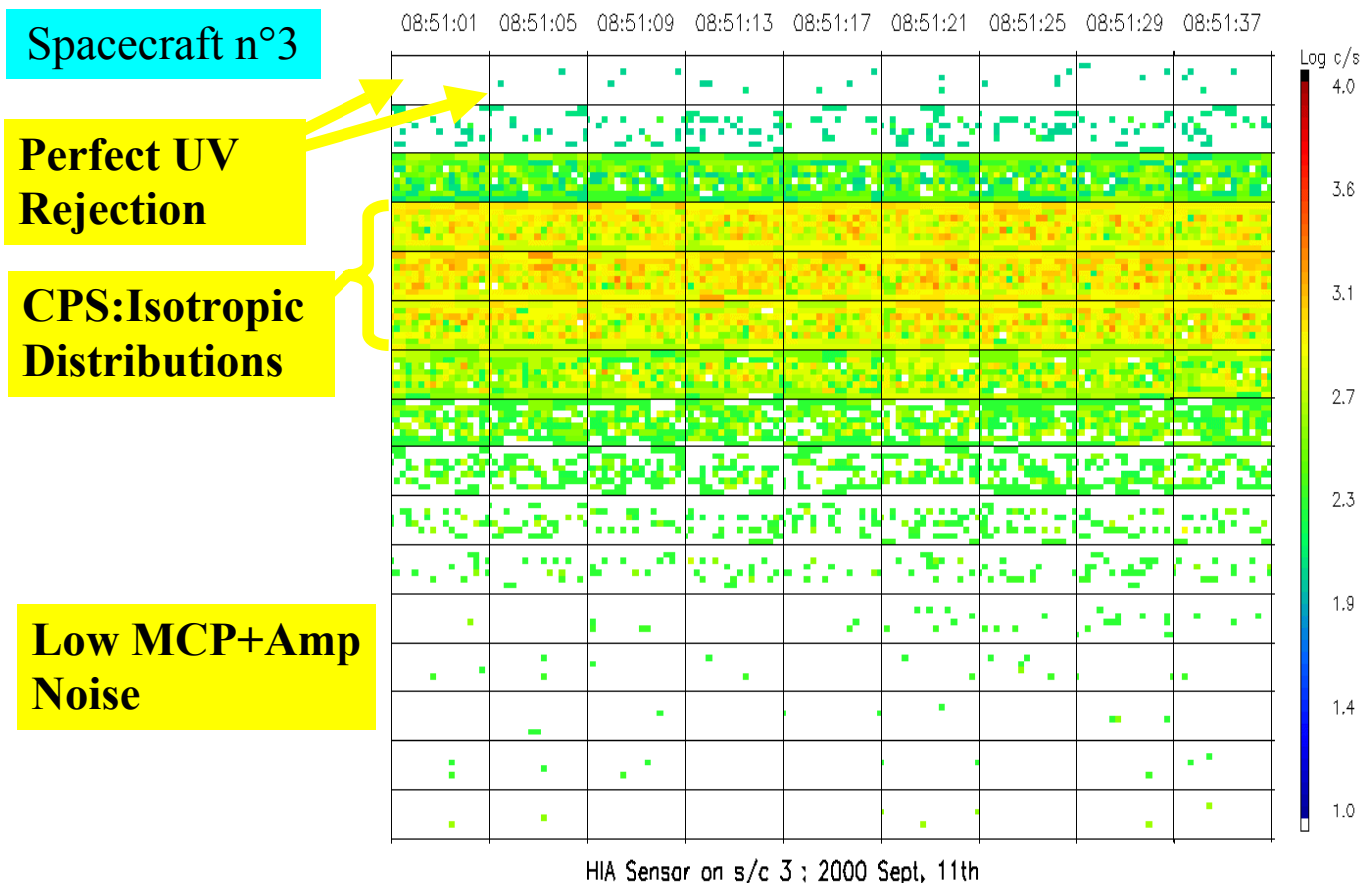

Fig. 8. Example of in-flight measurements of HIA on spacecraft 3 in the central plasma sheet on 11 September 2000. Each rectangle is a $\Theta-\Phi$ plot, $\Theta$, in ordinate, ranging from $-90^{\circ}$ to $+90, \Phi$, in abscissa, ranging from $180^{\circ}$ to $+180^{\circ}$, with the sunward direction at $(\Theta$, $\Phi)$ $=\left(0^{\circ}, \pm 180^{\circ}\right)$. Each line corresponds to 1 of the 16 logarithmically spaced energies between $24.3 \mathrm{eV}$ (bottom line) to $34117.3 \mathrm{eV}$. Ten successive measurements are shown (one by column). There is no Sun effect in the detector.

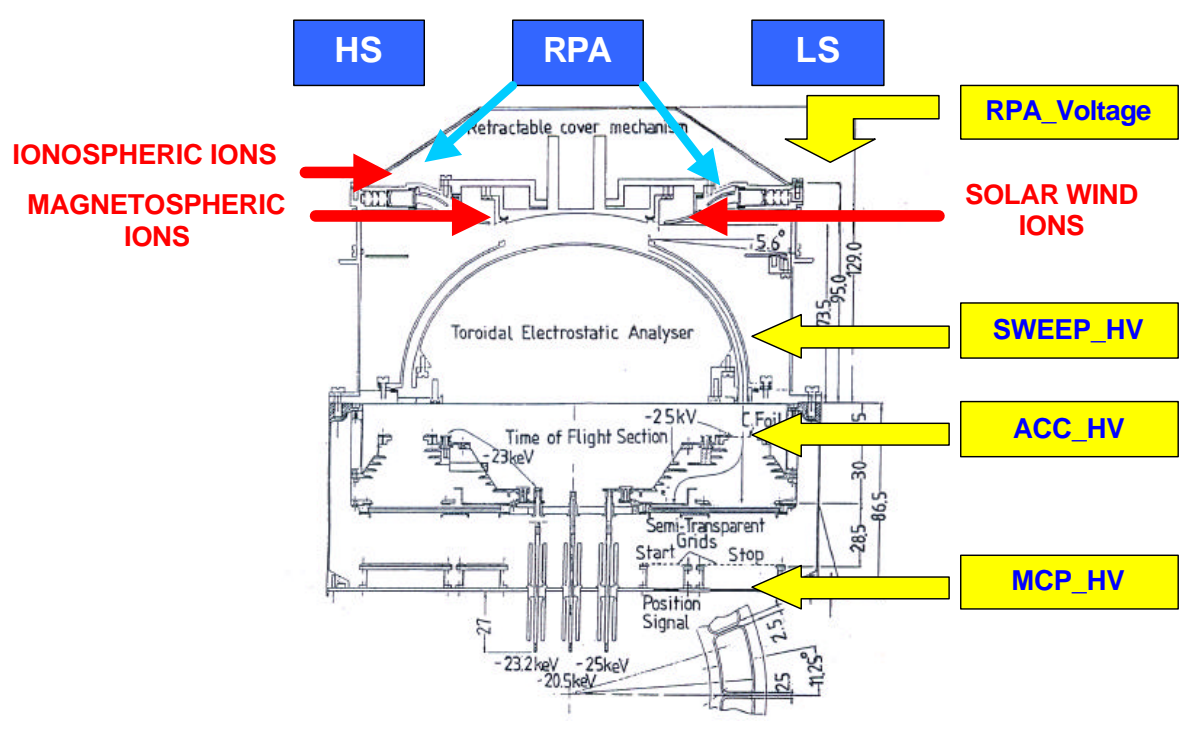

Fig. 9. Cross sectional view of the CODIF sensor. The voltages in the TOF section are shown for a $25 \mathrm{kV}$ post-acceleration.

a single sensitivity is only $4 \times 10^{3}$.

Figure 1 shows the fluxes covered by CODIF, ranging from magnetosheath/magnetopause protons to tail lobe ions (which consists of protons and heavier ions); fluxes from $\sim 10^{3}$ to over $10^{8}$ must be covered, requiring a dynamic range of larger than $10^{5}$. This can only be achieved if CODIF incorporates two sensitivities, differing by a factor of about 100. Therefore, CODIF consists of two sections, each with a $180^{\circ}$ field of view, with different (by a factor of 100) geometrical factors. In this way, one section always has count rates which are statistically meaningful and at the same time, the section can be handled by the time-of-flight electronics. The exception is solar wind $\mathrm{H}^{+}$which often saturates the instrument, but is measured with the small $g$ of HIA.

The CODIF instrument combines the ion energy per charge selection by deflection in a rotationally symmetric 


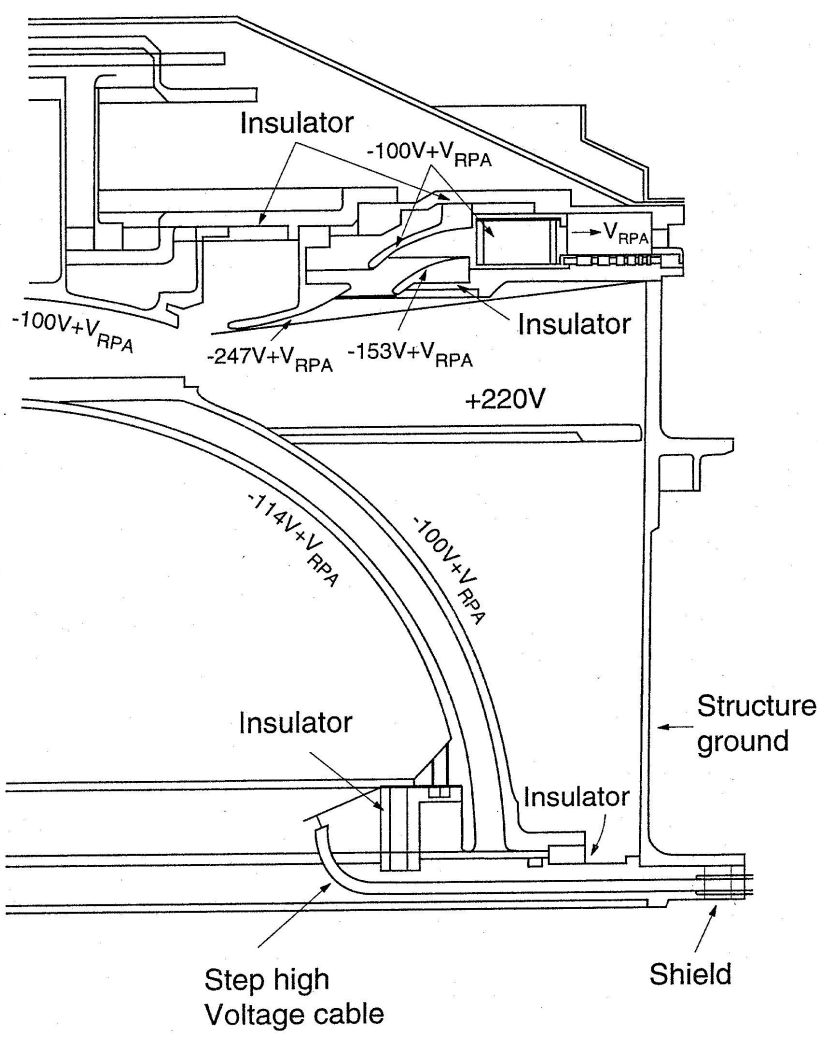

Fig. 10. Geometry of the CODIF RPA.

toroidal electrostatic analyser with a subsequent time-offlight analysis after post-acceleration to $\geq 15 \mathrm{keV} / \mathrm{e}$. A cross section of the sensor showing the basic principles of operation is presented in Fig. 9. The energy-per-charge analyser is of a rotationally symmetric toroidal type, which is basically similar to the quadrispheric top-hat analyser used for HIA. It has a uniform response over $360^{\circ}$ of the polar angle. The energy per charge selected by the electrostatic analyser $E / Q$, combined with the energy gained by post-acceleration e. $U_{A C C}$, and the measured time-of-flight through the length $d$ of the time-of-flight (TOF) unit, $\tau$, yield the mass per charge of the ion $M / Q$ according to:

$M / Q=2\left(E / Q+e \cdot U_{\mathrm{ACC}}\right) /(d / \tau)^{2} \cdot \alpha$.

The quantity $\alpha$ represents the effect of energy loss in the thin carbon foil $\left(\sim 3 \mu \mathrm{g} \mathrm{cm}^{-2}\right)$ at the entry of the TOF section and this depends on the particle species and incident energy.

\subsection{Electrostatic analyser description}

The electrostatic analyser (ESA) has a toroidal geometry which provides optimal imaging just past the ESA exit. This property was first demonstrated by Young et al. (1988). The ESA consists of inner and outer analyser deflectors, a tophat cover and a collimator. The inner deflector consists of toroidal and spherical sections which join at the outer deflector entrance opening (angle of $17.9^{\circ}$ ). The spherical section has a radius of $100 \mathrm{~mm}$ and extends from 0 to $17.9^{\circ}$ about the $Z$-axis. The toroidal section has a radius of $61 \mathrm{~mm}$ in the poloidal plane and extends from $17.9^{\circ}$ to $90^{\circ}$. The outer deflector covers the toroidal section and has a radius of $65 \mathrm{~mm}$. The top-hat cover consists of a spherical section with a radius of $113.2 \mathrm{~mm}$, which extends from 0 to $16.2^{\circ}$. Therefore, fits inside the entrance aperture of the outer deflector. The outer deflector and the top-hat cover are at signal ground under normal operation, but are biased at about $-100 \mathrm{~V}$ during RPA operation. The inner deflector is biased with voltages varying from -1.9 to $-4950 \mathrm{~V}$ in order to cover the energy range in a normal ESA operation. These are set to about $-113 \mathrm{~V}$ for the RPA.

The fact that the analyser has a complete cylindrical symmetry provides the uniform response in the polar angle. A beam of parallel ion trajectories is focused to a certain location at the exit plane of the analyser. The exit position, and thus the incident polar angle of the ions, is identified by using the information from the start detector (see Sect. 3.2). The full angular range of the analyser is divided into 16 channels of $22.5^{\circ}$ each. The broadening of the focus at the entrance of the TOF section is small compared to the width of the angular channels.

As illustrated in Fig. 9, the analyser is surrounded by a cylindrical collimator which serves to define the acceptance angles and restricts UV light. The collimator consists of a cylindrical can with an inner radius of $96 \mathrm{~mm}$. The entrance is covered by an attenuation grid with a radius of $98 \mathrm{~mm}$ which is kept at spacecraft ground. The grid has a $1 \%$ transmission factor over $50 \%$ of the analyser entrance and $>95 \%$ transmission over the remaining $50 \%$. The high transmission portion extends over the azimuthal angle range of $0^{\circ}$ to $180^{\circ}$ where $0^{\circ}$ is defined along the spacecraft spin axis. The low transmission portion, whose active entrance only extends from $22.5^{\circ}$ to $157.5^{\circ}$ in order to avoid the counting of any crossover from the other half, has a geometric factor that is reduced by a factor of $\approx 100$ in order to extend the dynamic range to higher flux levels. On the low-sensitivity half, the collimator consists of a series of 12 small holes, vertically spaced by approximately $1.9^{\circ}$ around the cylinder. These apertures have acceptance angles of $5^{\circ} \mathrm{FWHM}$, so there are no gaps in the polar angle coverage. The ion distributions near the polar axis are highly over-sampled during one spin relative to the equatorial portion of the aperture. Therefore, count rates must be weighted by the sine of the polar angle to normalise the solid-angle sampling for the moment calculations and 3D distributions.

The analyser has a characteristic energy response of about 7.6, and an intrinsic energy resolution of $\Delta E / E \cong 0.16$. The entrance fan covers a viewing angle of $360^{\circ}$ in the polar angle and $8^{\circ}$ in the azimuth. With an analyser voltage of $1.9-4950 \mathrm{~V}$, the energy range for ions is $15-38000 \mathrm{eV} / \mathrm{e}$. The deflection voltage is varied in an exponential sweep. The full energy sweep with 30 contiguous energy channels is performed 32 times per spin. Thus, a partial two-dimensional cut through the distribution function in the polar angle is obtained every $1 / 32$ of the spacecraft spin. The full $4 \pi$ ion distributions are obtained in a spacecraft spin period. 


\section{Schematics of CODIF}

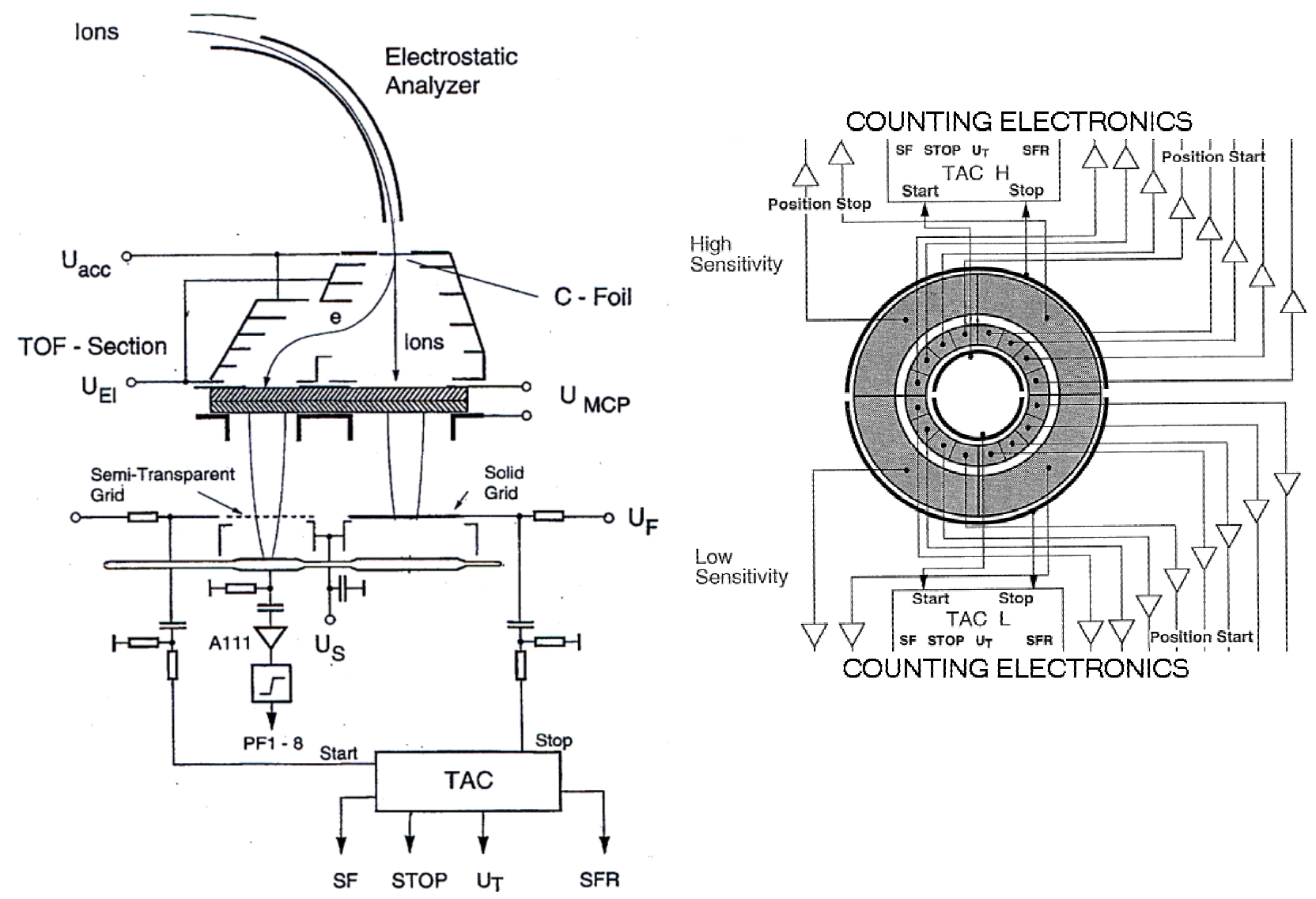

Fig. 11. The CODIF sensor: schematics (left) and MCP sectoring (right).

The outer plate of the analyser is serrated in order to minimize the transmission of scattered ions and UV, for the same reason the analyser plates are covered with a copper black coating. Behind the analyser, the ions are accelerated by a post-acceleration voltage of -14 to $-25 \mathrm{kV}$, such that thermal ions also have sufficient energy before entering the TOF section. After the first in-flight tests, this high voltage has been set at $-15 \mathrm{kV}$ in all of the spacecraft, giving good results and safe use of CODIF.

\subsection{Retarding Potential Analyser}

In order to extend the energy range of the CODIF sensor to energies below $15 \mathrm{eV} / \mathrm{e}$, an RPA assembly is incorporated in the two CODIF apertures (see Fig. 10). The RPA provides a way of selecting low-energy ions as input to the CODIF analyser without requiring the ESA inner deflector to be set accurately near $0 \mathrm{~V}$. The RPA collimates the ions, provides a sharp low-energy cutoff at a normal incident grid, pre-accelerates the ions to $100 \mathrm{eV}$ after the grid, and deflects the ions into the ESA entrance aperture. The energy pass of the ESA is about $5-6 \mathrm{eV}$ at $100 \mathrm{eV}$ of pre-acceleration, assuming all deflection voltages are optimised. This energy pass is very sensitive to the actual RPA deflection optics, so that deflection voltages have to be determined at about the $1 \%$ level.
The RPA assembly consists of a collimator, an RPA grid and pre-acceleration region, and deflection plates. The collimator section is kept at spacecraft ground. When the RPA is active, only RPA measurements are produced by CODIF. The RPA can be thought of as a separate ion optics front end for CODIF, which can be used in on command, thereby replacing the normal ion optics. A separate RPA aperture ring defines a field of view parallel to the normal CODIF field of view, but displaced towards the analyser top by about $15 \mathrm{~mm}$. As with normal CODIF operations, the field of view extends $180^{\circ}$ in azimuth on one side of the analyser and $135^{\circ}$ on the other side. Only one side can be active at a time. Unlike the normal CODIF entrance aperture, both sides of the RPA have the same sensitivity; there is no attenuation grid on one half to reduce the effective geometric factor for the RPA.

When the RPA is enabled, the normal entrance aperture is closed off by a positively biased grid, which pushes ions near $100 \mathrm{eV} / \mathrm{e}$ away from the entrance slot below the top cap. Although higher energy ions could still enter this slot, the bias between hemispheres is set to pass energies only near $100 \mathrm{eV} / \mathrm{e}$, so that higher energy ions strike the inner hemisphere, and fail to traverse the analyser gap to exit the ring. A retarding grid at the RPA entrance rejects ions with energy/charge below the set threshold voltage and allows 


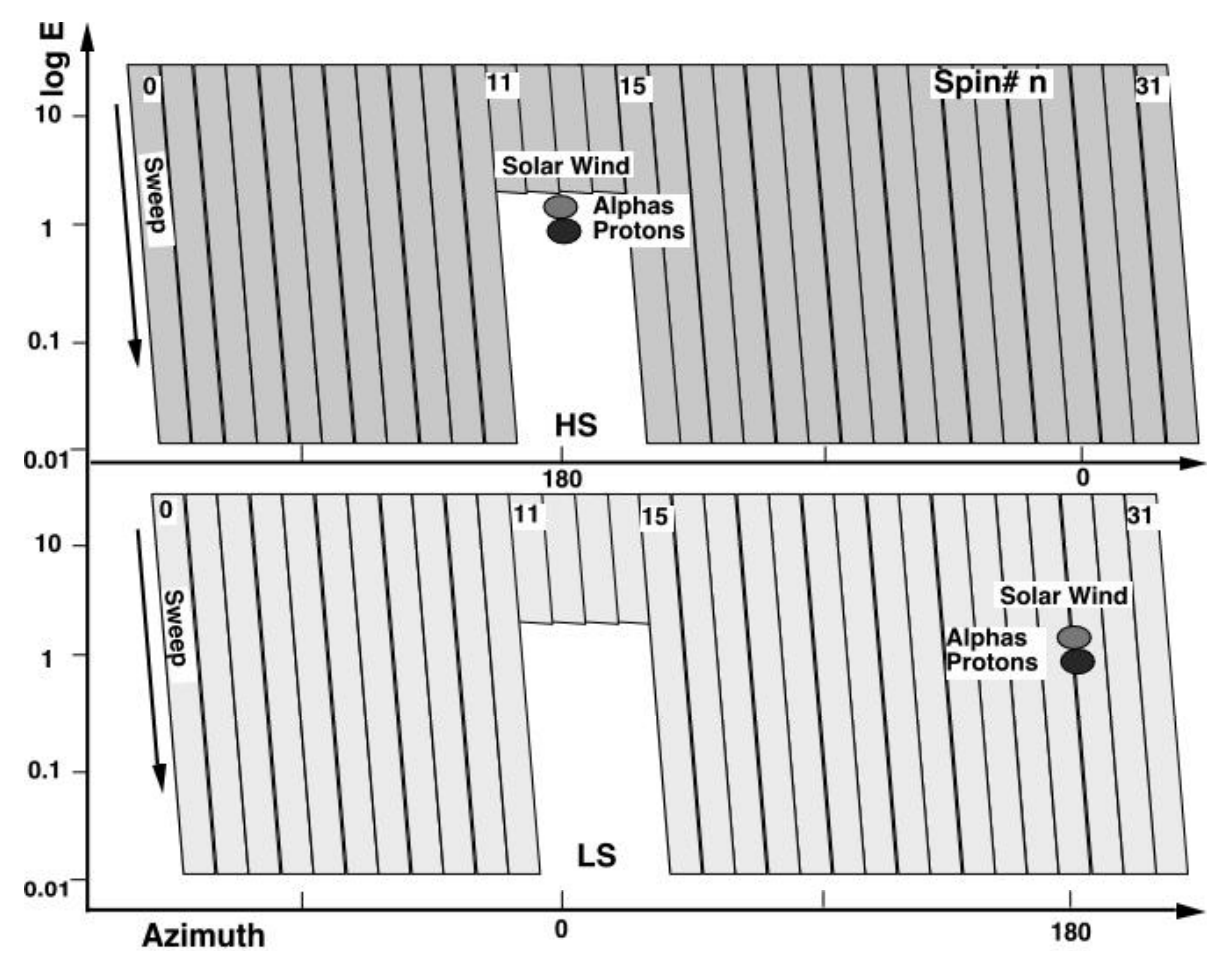

Fig. 12. Energy sweeping scheme of CODIF in the solar wind. The sweep is shown in the $\log E$ versus the azimuthal angle for the highsensitivity section (upper panel) and low-sensitivity section (lower panel), starting at the high energy end. When looking into the solar wind, the sweep stops above the alpha particles for the high-sensitivity section but the sweep does not stop for the alpha particles and the protons for the low-sensitivity section.

higher energy/charge ions to pass. The accepted ions are first collimated and accelerated by 100 volts, and then routed by three deflector surfaces into the main entrance slot. The hemispherical analyser filters out the higher energies from the incoming beam and the remainder enter the TOF section for a velocity measurement. The deflection system provides a method of steering the RPA low-energy ions into the CODIF ESA.

The RPA grid and pre-acceleration region consist of a pair of cylindrical rings, sandwiched between resistive ceramic material. Both inner and outer cylindrical rings contain apertures separated by posts every $22.5^{\circ}$, similar to the ESA collimator entrance, in order to allow the ions to pass through the assembly. The RPA grid is attached to the inner surface of the outer cylindrical ring. This outer ring has a small ledge which captures the RPA grid and which also provides the initial optical lens that is crucial to the RPA operation. Both inner and outer cylindrical rings are in good electrical contact with the resistive kapton (silver epoxy). During RPA operation, the outer cylindrical ring is biased from spacecraft ground to about $+25 \mathrm{~V}$, and provides the sharp, low-energy RPA cutoff. This voltage is designated $V_{r p a}$ in Fig. 10. The inner cylindrical ring tracks the outer ring voltage and is biased at $-100 V+V_{\text {rpa }}$. The inner cylindrical ring, the ESA outer deflector, and the ESA top-hat cover are electrically tied to the RPA deflector.

The RPA deflection plates consist of three toroidal deflec- tors located above the ESA collimator entrance and one deflector disk located below the collimator entrance. The three toroidal deflectors are used to deflect the ions into the ESA. The deflector disk is used to prevent low-energy ions from entering the main aperture and to collect any photoelectrons produced inside the analyser, while in RPA mode.

\subsection{Time-of-flight and detection system}

The CODIF sensor uses a time-of-flight technology (Möbius et al., 1985). The specific parameters of the time-of-flight spectrometer have been chosen such that a high detection efficiency of the ions is guaranteed. High efficiency is not only important for maximizing the overall sensor sensitivity, but it is especially important for minimising false mass identification resulting from false coincidence at a high counting rate. A carbon foil, that is too thin, would result in a significant reduction in the efficiency of secondary electron production for the "start" signal, while an increase in thickness does not change the secondary electron emission significantly (Ritter, 1985). Under these conditions, a post-acceleration of $\geq 14 \mathrm{kV}$ is necessary for the mass resolution of the sensor.

After passing the ESA, the ions are focused onto a plane close to the entrance foil of the time-of-flight section (Fig. 11). The TOF section is held at the post-acceleration potential in order to accelerate the ions into the TOF section, where the velocity of the incoming ions is measured. The flight path of the ions is defined by the $3 \mathrm{~cm}$ distance between 

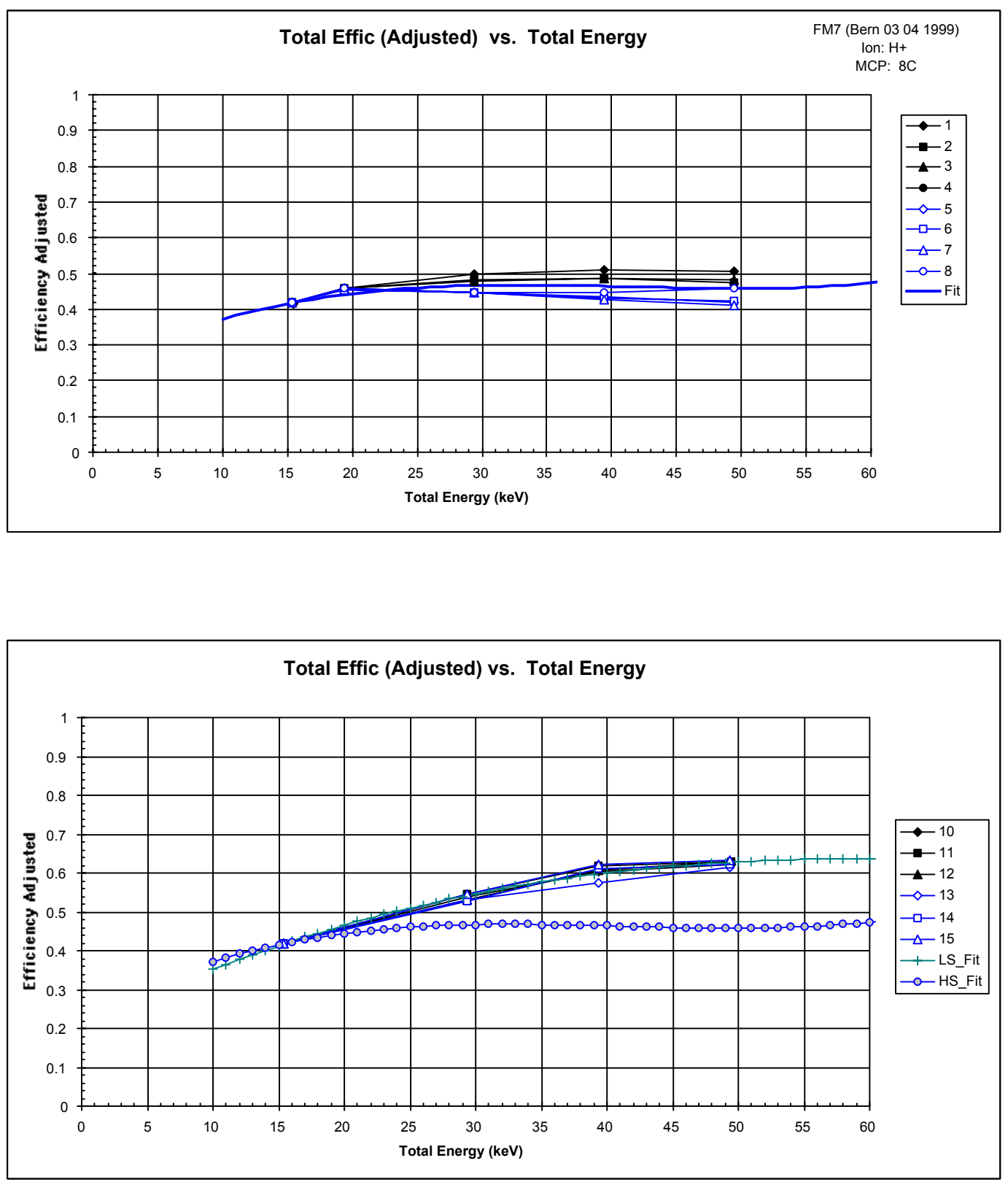

Fig. 13. CODIF FM7 High Side normalised total efficiency verses total energy for $\mathrm{H}^{+}$for the different anodes and with a general fit (upper curve) and CODIF FM7 Low Side normalised total efficiency verses total energy for $\mathrm{H}^{+}$for the different anodes and with a general fit; High Side fit of the upper curve is added (lower curve).

the carbon foil at the entrance and the surface of the "stop" microchannel plate (MCP). The start signal is provided by secondary electrons, which are emitted from the carbon foil during the passage of the ions. The entrance window of the TOF section is a $3 \mu \mathrm{g} \mathrm{cm}^{-2}$ carbon foil, which has an optimum thickness between the needs of low-energy loss and straggling in the foil, and high efficiency for secondary electron production. The electrons are accelerated to $2 \mathrm{keV}$ and deflected onto the start MCP assembly by a suitable potential configuration.

The secondary electrons also provide the position information for the angular sectoring. The carbon foil is made up of separate $22.5^{\circ}$ sectors, separated by narrow metal strips. The electron optics are designed to strongly focus secondary electrons, originating at a foil, onto the corresponding MCP start sector.

The MCP assemblies (Fig. 11) are ring-shaped with inner and outer radii of $6 \times 9 \mathrm{~cm}$ and $3 \times 5 \mathrm{~cm}$ for the stop and start detectors, respectively. For the start signals, the output of the MCPs is collected on a set of segmented plates behind the MCPs $\left(22.5^{\circ}\right.$ each), and on thin wire grids with $\approx 50 \%$ transmission at a distance of $10 \mathrm{~mm}$ in front of the signal plates. The stop signals are collected through a solid, non-transparent grid (and not through a semitransparent grid, 

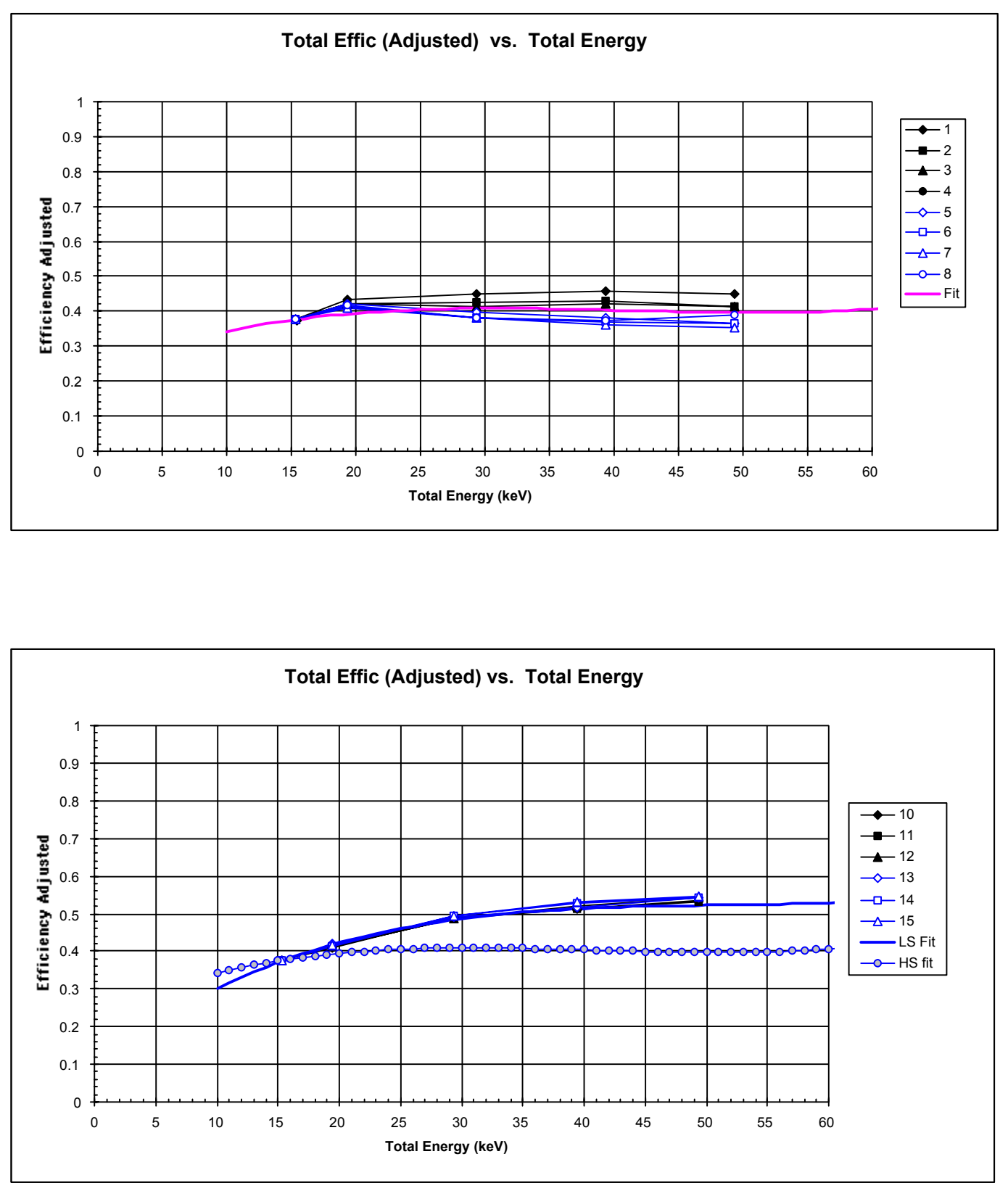

Fig. 14. CODIF FM7 High Side normalised total efficiency verses total energy for $\mathrm{He}^{+}$for the different anodes and with a general fit (upper curve) and CODIF FM7 Low Side normalised total efficiency verses total energy for $\mathrm{He}^{+}$for the different anodes and with a general fit; High Side fit of the upper curve is added (lower curve).

such as for Cluster-1), improving significantly the $\mathrm{H}^{+}$detection efficiency. All are at ground potential (see Fig. 9). Thus, almost all of the post-acceleration voltage is applied between the rear side of the MCPs and the signal anodes. The timing signals are derived from the $50 \%$ transmission grids, and separately derived for the high- and the low-sensitivity TOF section. The position signals, providing the angular information in terms of $22.5^{\circ}$ sectors, are derived from the signal plates behind the start MCP. The main performances of the CODIF sensor are summarised in Table 2.

\subsection{Sensor electronics}

The sensor electronics of the instrument consist of two timeto-amplitude converters (TACs) to measure the time-of-flight of the ions between the start carbon foil and the stop MCPs, two sets of eight position discriminators at the start MCPs, two sets of two position discriminators at the stop MCPs, and the event selection logic. Each individual ion is pulseheight-analysed according to its time-of-flight incidence in azimuthal (given by the spacecraft spin) and the polar angle (given by the start position), and the actual deflection voltage. 

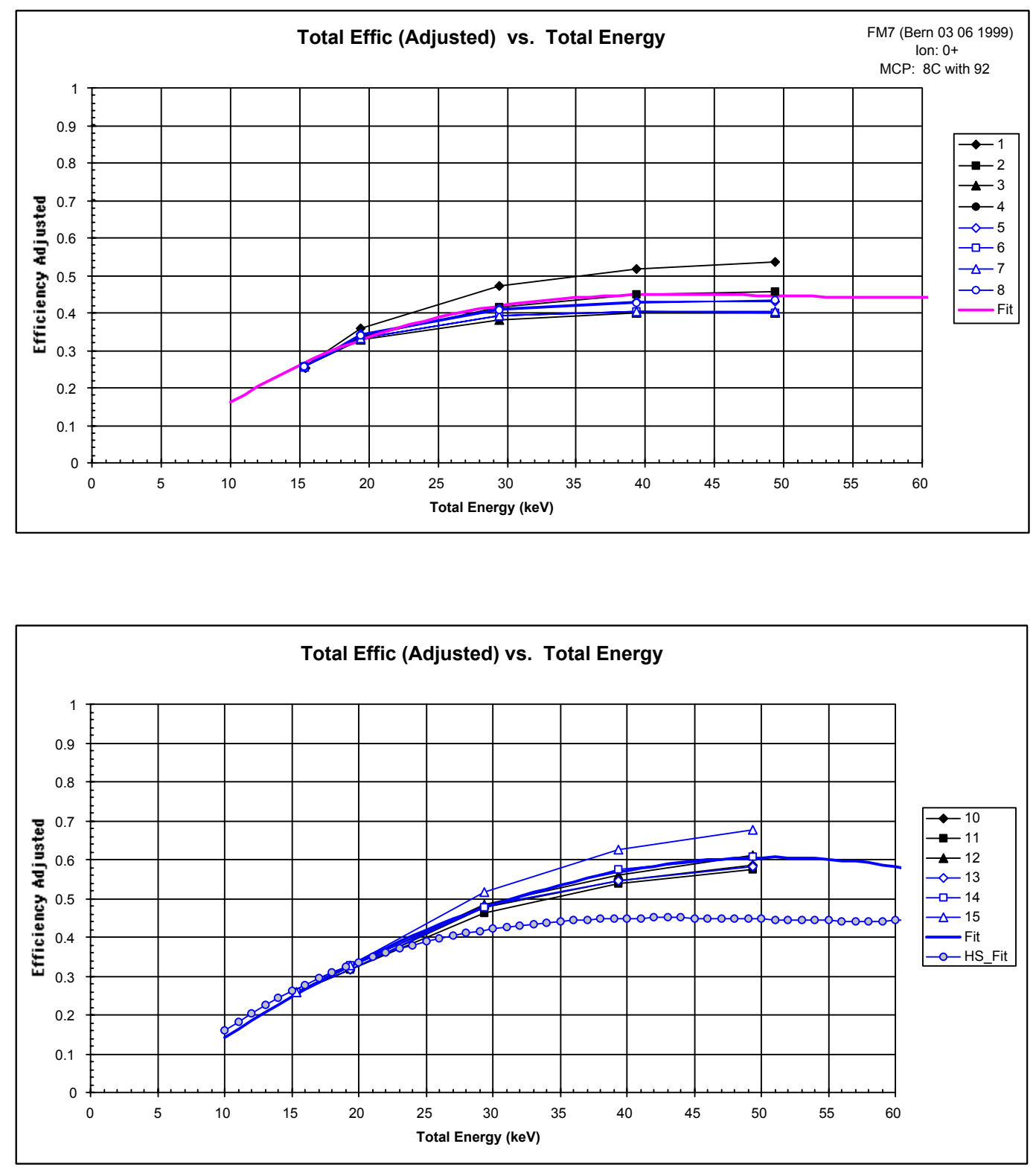

Fig. 15. CODIF FM7 High Side normalized total efficiency verses total energy for $\mathrm{O}^{+}$for the different anodes and with a general fit (upper curve) and CODIF FM7 Low Side normalised total efficiency verses total energy for $\mathrm{O}^{+}$for the different anodes and with a general fit; High Side fit of the upper curve is added (lower curve).

The eight position signals for each TOF section (one TOF section for the Low Side, one TOF for the High Side, see Fig. 11), in order to achieve the $22.5^{\circ}$ resolution in the polar angle, are independently derived from the signal anodes, while the timing signals are taken from the grids in front of the anodes. Likewise, the stop MCPs, consisting of four individual MCPs, are treated separately to carry along partial redundancy. By this technique, the TOF and the position signals are electrically separate in the sensor. The position pulses are fed into charge-sensitive amplifiers and identified by pulse discriminators, the signal of which is directly fed into the event selection logic. The TOF unit is divided into two TOF channels.
The conditions for valid events are established in the event-selection logic. The respective coincidence conditions can be changed via ground command. Several count rates are accumulated in the sensor electronics. There are monitor rates of the individual start and stop detectors to allow for the continuous monitoring of the carbon foil and MCP performance. The total count rates of TOF coincidence show the valid events accumulated for each TOF section. These rates can be compared with the total stop count rates in order to monitor in-flight the efficiency of the start and stop assemblies.

In order to protect the MCPs, the solar wind protons and the solar wind alpha particles are blocked from detection by 

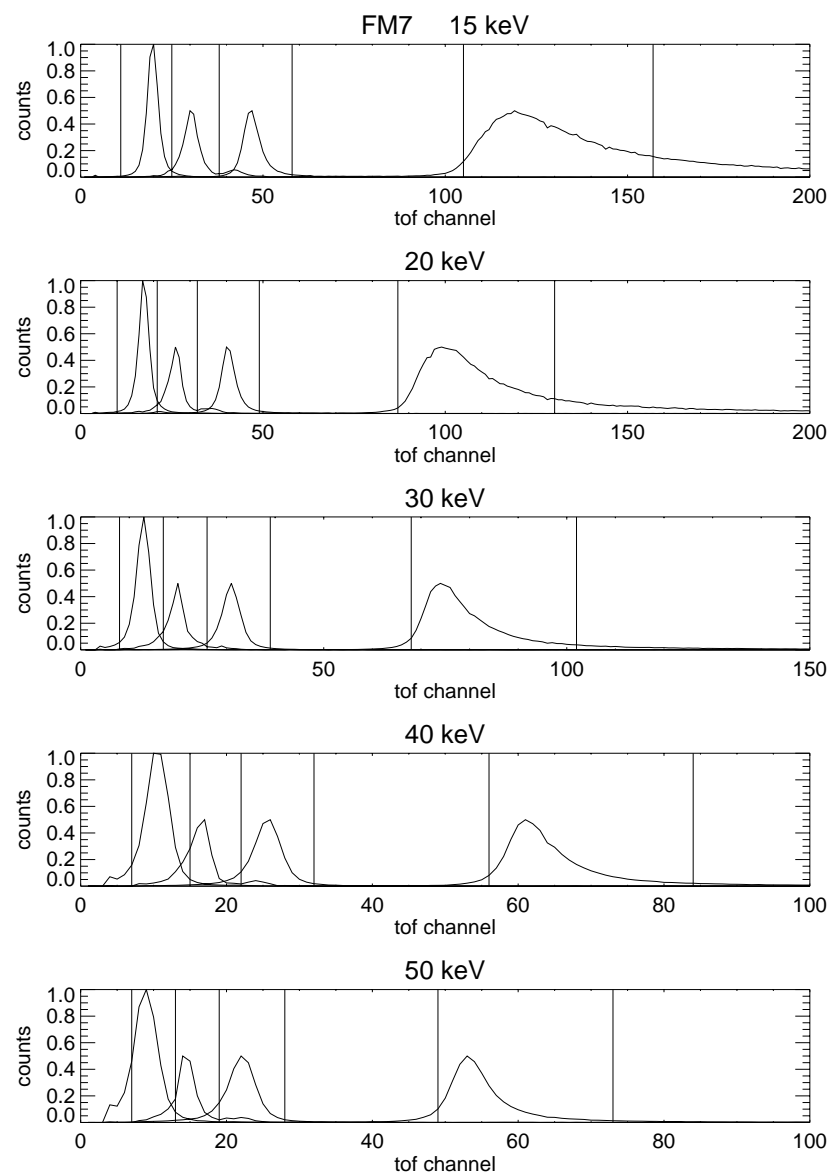

Fig. 16. CODIF FM7 time-of flight spectra for the four major species at 5 energies. The spectra are averaged over all positions. The vertical lines show the thresholds used to distinguish species.

a simple scheme during the sweeping cycle, as shown in Fig. 12 (actually there are four consecutive sweeps that are modified when $G$ is facing the solar wind, whereas they are not modified when $\mathrm{g}$ is facing the solar wind). The sweep, starting at high energies, is shown for the high-sensitivity section in the upper panel and for the low-sensitivity section in the lower panel in $\log E$ and the azimuthal angle. The voltage sweep, which starts at high energies, is stopped above the alphas when the high-sensitivity section is facing the solar wind. The result is a small data gap for both sections of the sensor simultaneously. The primary purpose for introducing this scheme is to avoid a short-time gain depression of the MCP area, which would otherwise persist on the order of $1 \mathrm{~s}$ after the impulsive high count rate that would result from the solar wind.

\subsubsection{High voltage system}

A sweep-voltage, high-voltage power supply generates an exponential voltage waveform from 1.9 to $4950 \mathrm{~V}$ for the electrostatic analyser. A $\geq 14 \mathrm{kV}$ static supply feeds the postacceleration voltage, which can be adjusted via ground command. Another adjustable supply is used for the MCPs and
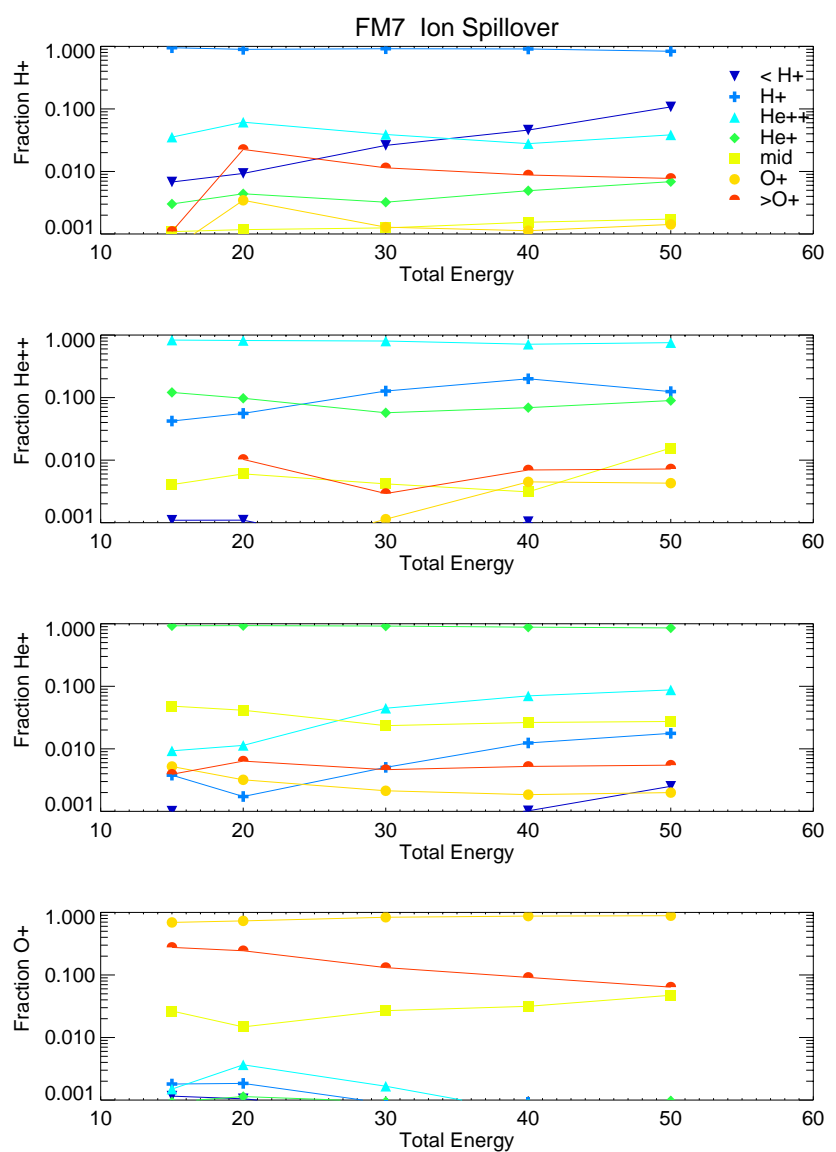

Fig. 17. Example of ion spillover effect in CODIF FM7: fraction of each 4 major ion species detected in the $\mathrm{H}^{+}, \mathrm{He}^{++}, \mathrm{He}^{+}$, and $\mathrm{O}^{+}$ bins.

the collection of secondary electrons. It supplies up to $5 \mathrm{kV}$ and is floated on top of the post-acceleration voltage.

\subsection{In-flight calibration}

Upon command, an in-flight-calibration (IFC) pulse generator can stimulate the two independent TOF branches of the electronics according to a predefined program. Within this program, all important functions of the sensor electronics and the subsequent on board processing of the data can be automatically tested. Temporal variations of calibration parameters can be measured. The in-flight calibration can also be triggered by ground command in a very flexible way, e.g. for trouble shooting purposes. In addition, the known prominent location of the proton signal can, if necessary, serve as a tracer of changes in the sensor itself.

\subsection{CODIF performances}

\subsubsection{Resolution in mass per charge}

The instrumental resolution in mass per charge is determined by a combination of the following effects: 

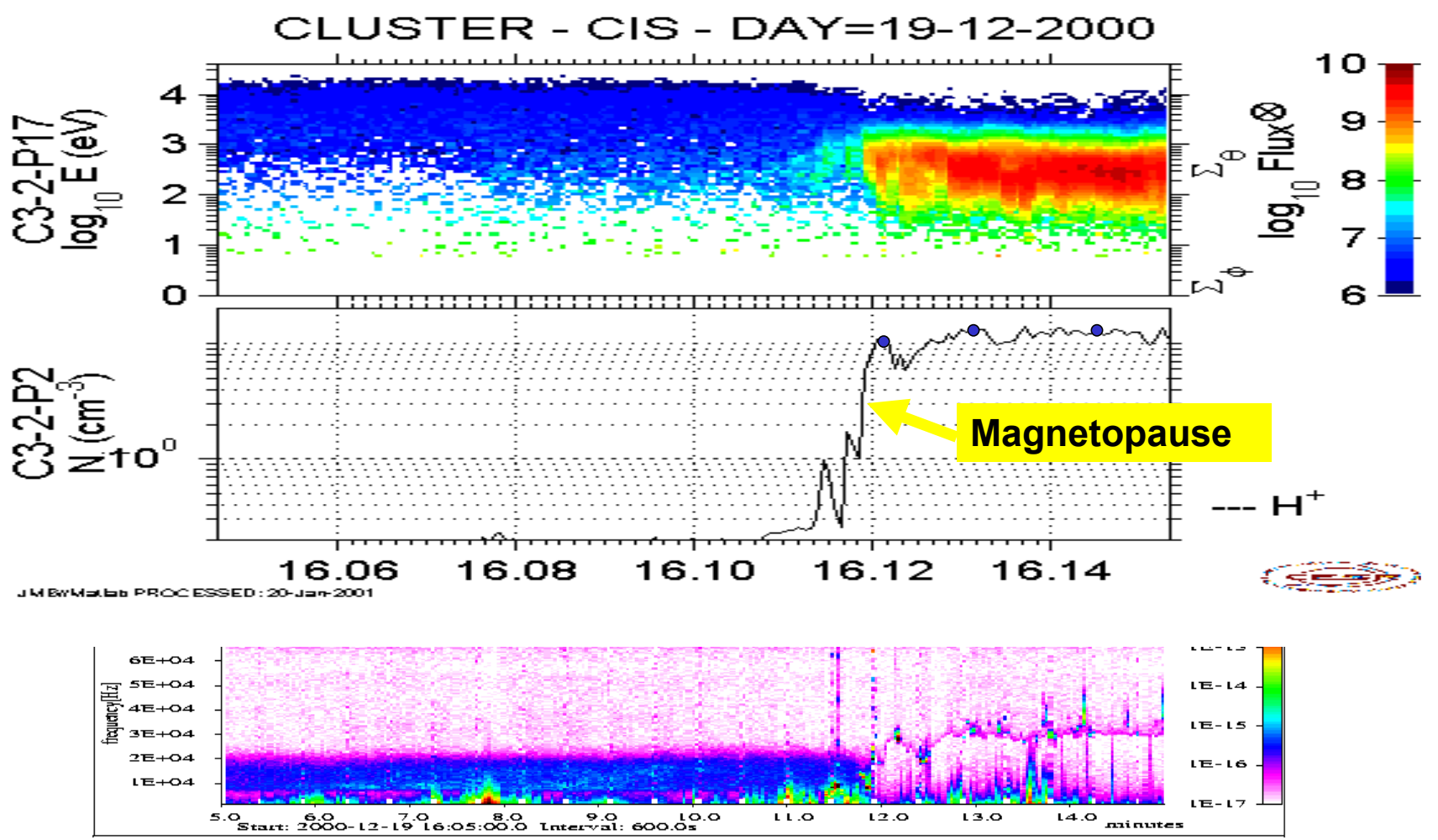

Fig. 18. Comparison of the electron density measured by the Whisper instrument and the ion density measured by HIA, on 19 December 2000 when the spacecraft 3 leaves the magnetosphere and moves into the magnetosheath. At the top are the ion fluxes, at the bottom are the wave measurements of Whisper and in the middle is the density measured by HIA and three-points deduced from the Whisper measurements. The agreement is excellent. Before the magnetopause traversal, the density is too small to be evaluated by Whisper.

- Energy resolution of the electrostatic deflection analyser $(\Delta E / E=0.16)$

- TOF dispersion caused by the angular spread of the ion trajectories due to the characteristics of the analyser and the straggling in the carbon foil (the angular spread of $=$ $13^{\circ}$ leads to $\Delta \tau / \tau=0.03$ );

- TOF dispersion caused by energy straggling in the carbon foil $(\Delta \tau / \tau)$ up to 0.08 for $\left.25 \mathrm{keV} \mathrm{O}^{+}\right)$;

- Electronic noise in the TOF electronics and secondaryelectron flight time dispersion (typically $0.3 \mathrm{~ns}$ ).

The resulting TOF dispersion amount, $(\Delta \tau / \tau) \leq 0.1$, finally leads to a $M / Q$ resolution between 0.15 for $\mathrm{H}^{+}$, and 0.25 for low energy $\mathrm{O}^{+}$.

\subsubsection{CODIF calibrations}

The TOF efficiency is a function of the ion species and the total energy, which is the sum of the original ion energy plus the energy gained in the post-acceleration potential. The total efficiency for measuring an ion in CODIF is determined by the efficiency of the "Start" signal, the efficiency of the "Stop" signal, and the efficiency of "Valid Single Events". The "Start" efficiency is a function of the number of secondary electrons emitted from the carbon foil, the focusing of the electrons onto the MCP, the MCP active area, and the MCP gain and MCP signal threshold. It is measured using the ratio of the Start-Stop Coincidence rate (SFR) to the "Stop" rate (SR). The "Stop" efficiency is a function of the scattering of the ion in the foil (which can scatter it away from the active area), the MCP active area, and the MCP gain and signal threshold. It is given by the ratio of the SFR rate to the "Start" rate, SF. In order for an ion to be counted as a valid event, it must generate not only a start and stop signal, but also a single "Start Position" (PF) signal. The "Valid Event Efficiency" is given by the ratio of the Valid Single Event rate, SEV, to the SFR. These efficiencies are all a function of energy and species, as well as MCP voltage. Determining the final efficiencies is done in two steps. First, the optimum voltage at which to run the MCPs is determined. Then, using the optimum MCP voltage, the efficiencies for each species as a function of energy and position are determined.

Each CODIF model, including the spare model, has been very well calibrated (see Table 4). The results for CODIF CIS model FM7, on spacecraft 4, are presented here as an example. For the other models, see the full report of Kistler (2000).

To determine ion efficiencies verses energy, once the optimum MCP voltage is set, data are collected over a range of beam energies. The total ion efficiency is a function of total ion energy (original beam energy plus post-acceleration). 
Table 4. Summary of the geometric factors for one instrument position (out of 8 for High Side and 6 for Low Side), and energy and angle response for the CODIF instruments deduced from all calibration data

\begin{tabular}{ccccc}
\hline Model & $\begin{array}{c}\text { High Side } \\
\text { Geometrical } \\
\text { Factor* } \\
\text { (averaged) } \\
\mathrm{cm}^{2} \mathrm{sr}\end{array}$ & $\begin{array}{c}\text { Low Side } \\
\text { Geometrical } \\
\text { Factor* } \\
\text { (averaged) } \\
\mathrm{cm}^{2} \mathrm{sr}\end{array}$ & $\begin{array}{c}\mathrm{k} \\
\text { Analyser } \\
\text { Constant }\end{array}$ & $\begin{array}{c}\Delta \alpha \\
\text { (full) } \\
\text { FWHM } \\
\text { (degrees) }\end{array}$ \\
\hline FM5 & $2.58 \mathrm{E}-03$ & & 7.382 & 6.124 \\
FM6 & $2.45 \mathrm{E}-03$ & & 7.217 & 5.774 \\
FM7 & $2.53 \mathrm{E}-03$ & $2.36 \mathrm{E}-05$ & 7.412 & 5.790 \\
FM8 & $1.85 \mathrm{E}-03$ & $2.61 \mathrm{E}-05$ & 7.455 & 6.271 \\
FM4 (spare) & $2.45 \mathrm{E}-03$ & $2.74 \mathrm{E}-05$ & 7.566 & 6.136 \\
Average & $2.39 \mathrm{E}-03$ & $2.57 \mathrm{E}-05$ & & \\
\hline
\end{tabular}

Even when the instrument is operating at the optimum MCP voltage, there is a significant difference between the final efficiencies measured at different positions (pixels). Thus, it was necessary to determine the final ion efficiencies as a function not only of energies and species, but also of position.

Figures 13, 14 and 15 are plots of the total adjusted ion efficiencies verses total beam energy on both the High Side (HS) and Low Side (LS) for $\mathrm{H}^{+}, \mathrm{He}^{+}$, and $\mathrm{O}^{+}$ions. The efficiency for $\mathrm{He}^{++}$is the same as for $\mathrm{He}^{+}$at the same total energy (not energy per charge). Since $\mathrm{He}^{++}$goes to twice the energy, we did separate curve fits for $\mathrm{He}^{++}$(not shown) to assure that the curves were stable at higher energies.

Figure 16 shows the time-of-flight spectra over a range of energies for FM7. This figure is assembled from many data sets using individual species. The relative heights of the peaks depend on the beam intensity and length of the run, and, therefore, have no significance for this analysis. The vertical lines show the thresholds used to distinguish the species. During commissioning, it was found that a large peak can be observed in the lowest channel, and during time periods with a high oxygen flux, there is a second peak below the proton peak which seems to be correlated with the $\mathrm{O}^{+}$flux. It is probably due to ions with a time-of-flight greater than the allowed $200 \mathrm{~ns}$ from the long $\mathrm{O}^{+}$tail. To keep these spurious peaks from being counted with the protons, a threshold below the $\mathrm{H}^{+}$peak was introduced.

The mass resolution of the CODIF instrument is defined by the resolution in time-of-flight. The width of the peaks in time-of-flight is determined by the spread in energy that results from the energy loss in the carbon foil and any noise in the time-of-flight electronics. Since the energy loss is a statistical process, ions that enter the foil with one energy come out with a range of energies. The percentage of energy that is lost is the worst for low-energy ions and heavy ions. The electronic noise in the time-of-flight circuit is independent of ion energy. Since the loss in the carbon foil is a smaller fraction of the total energy, the peaks should become narrower with increasing energy. This is evident in the $\mathrm{O}^{+}$peaks, but not so clear for the lower mass peaks. One reason for this is that there is a significant difference between the locations of the peaks for different positions. Since the peaks move closer together with energy, but the width of the low mass ions does not significantly improve, there are more problems with overlapping peaks, and, therefore, worse mass resolution at high energies. The bin with the most overlap with other species is the $\mathrm{He}^{++}$bin. A quantitative analysis of the spill-over between bins is shown in Fig. 17. Each panel shows the fraction of a particular species that is classified in a particular mass bin. The thresholds were chosen to maximize the percentage of an ion that falls into the correct bin, but also to minimize the percentage of $\mathrm{H}^{+}$ions that fall into the wrong bin. This is particularly important at the $\mathrm{H}^{+} / \mathrm{He}^{++}$boundary. Since there is usually much more $\mathrm{H}^{+}$than $\mathrm{He}^{++}$in space plasmas, a small percentage of $\mathrm{H}^{+}$spilling into the $\mathrm{He}^{++}$bin can significantly effect the $\mathrm{He}^{++}$measurement. In this case, about $3.5 \%$ of the $\mathrm{H}^{+}$ions fall into the $\mathrm{He}^{++}$bin, and $70 \%$ of the $\mathrm{He}^{++}$ions are in the $\mathrm{He}^{++}$bin. For $\mathrm{O}^{+}$, the fraction that falls into the $\mathrm{O}^{+}$bin was kept low at low-energies in order to reduce the background in the bin. At $15 \mathrm{keV}$, the $\mathrm{O}^{+}$has a long tail extending to high TOF channels. The background from accidental coincidences in a bin is proportional to the number of TOF channels in the bin, so there is an advantage to keeping a narrow bin, even if some of the real signal is lost.

The RPA geometric factor and energy response has also been calibrated (McCarthy, 2000). For the group of 8 anodes when the high sensitivity side is enabled, the total RPA geometric factor is $3.0 \times 10^{-2} \mathrm{~cm}^{2}$.sr. It is $2.2 \times 10^{-2} \mathrm{~cm}^{2}$.sr for the group of 6 anodes when the low sensitivity side is enabled.

\subsubsection{Dynamic range}

The design of the electrostatic analyser guarantees a large geometrical factor in the high-sensitivity section $A . \Delta E / E . \Delta \tau . \pi=0.025 \mathrm{~cm}^{2} . \mathrm{sr}$. The energy bandwidth is $\Delta E / E=0.16$. The efficiency of the TOF unit is about 0.5. Differential energy fluxes as low as $\sim 3 \times$ $10^{3}$ ions s $\mathrm{sm}^{-1} \mathrm{sr}^{-1}$ can be detected by the instrument with the full time resolution of 1 spin period and about 5 counts of the energy ${ }^{-1}$ channel. The sensitivity is increased accordingly for longer integration time. Therefore, the dynamic range reaches seven decades. The upper flux limit of the instrument amounts to $3 \times 10^{9}$ ions s $\mathrm{s}^{-1} \mathrm{~cm}^{-2} \mathrm{sr}^{-1}$, which leads to a count rate of $10^{5}$ counts $\mathrm{s}^{-1}$ in one TOF unit (near the saturation of the analysing electronics) and still guarantees a mass density determination with better than a $10 \%$ accuracy for the reduced aperture geometry.

\section{Data processing system}

CIS data can be collected in a variety of modes with different bit-rates: $5527 \mathrm{bit} / \mathrm{s}$ in mode NM1 (normal mode), $6521 \mathrm{bit} / \mathrm{s}$ in mode NM2 (ion mode), $4503 \mathrm{bit} / \mathrm{s}$ in mode NM3 (electron mode, with the PEACE instruments having more bits 


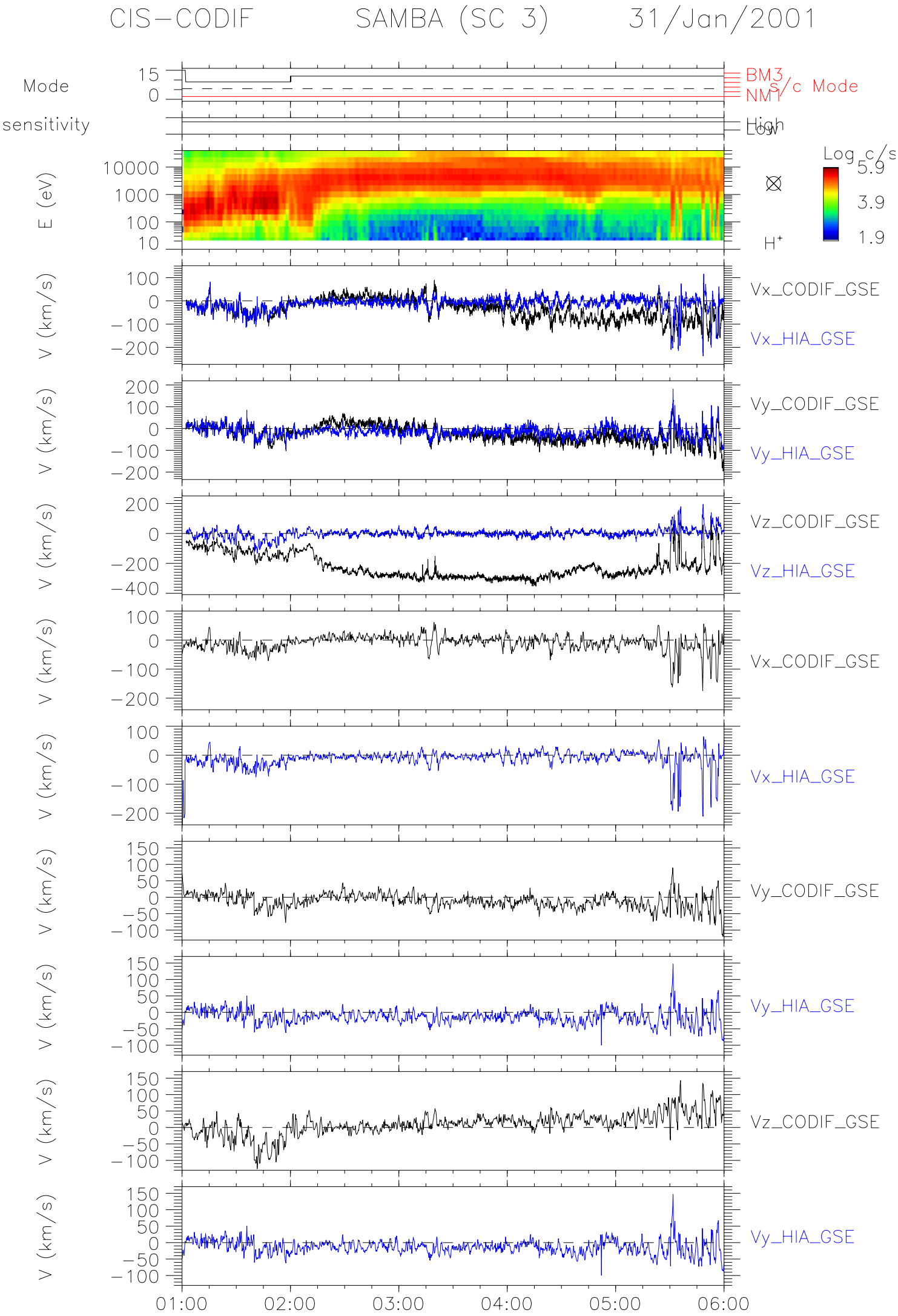

Fig. 19. Example showing the importance of the calibrations, from top to bottom: instrument modes, and CODIF and HIA sensitivities, energy-time spectrogram of CODIF for $\mathrm{H}^{+}$ions as a function of energy, on board calculated CODIF and HIA velocities (3 panels), velocities calculated on the ground using the 3D distribution functions, and correct efficiencies for HIA and CODIF (6 panels). 


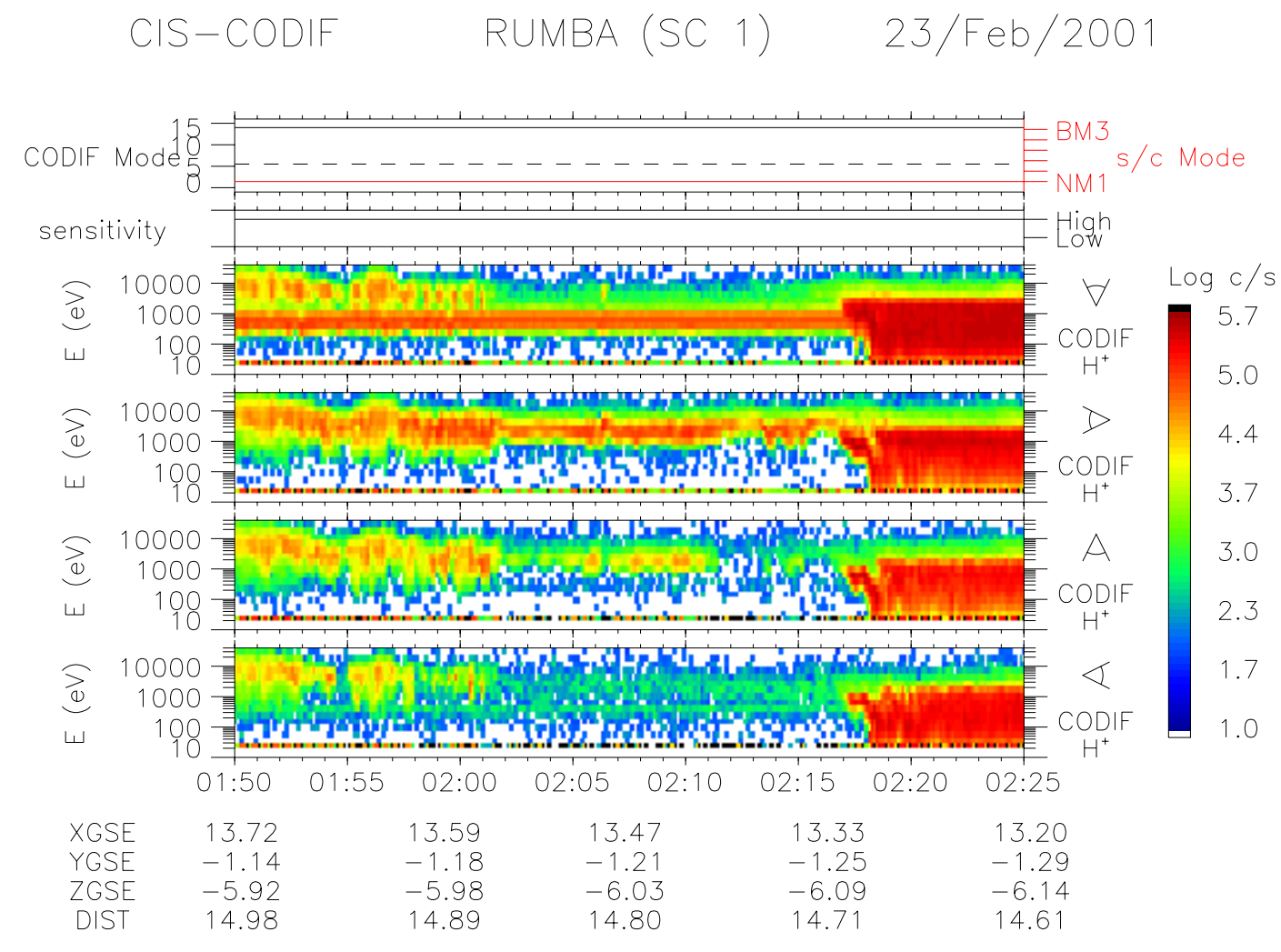
CIS-CODIF
SAMBA (SC 3)
$23 /$ Feb/2001

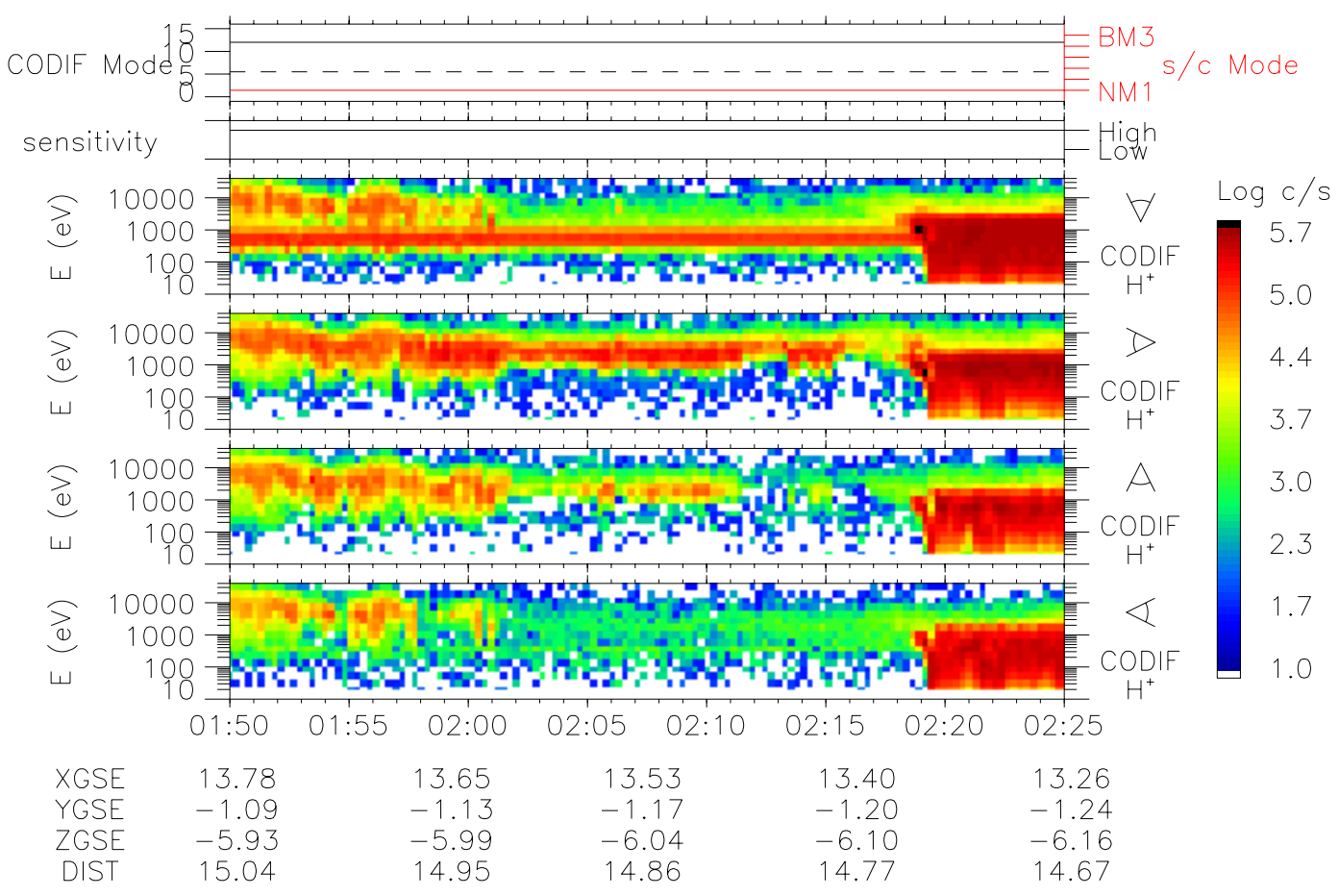

Fig. 20. Comparison of CODIF compressed data (upper panel) and uncompressed data (lower panel) on 23 February 2001: upper panel gives for spacecraft 1 , from top to bottom, the telemetry modes, sensitivity and uncompressed energy-time spectrogram $\mathrm{H}^{+}$CODIF in four directions (top to bottom: sunward, dusk, anti-sunward and dawn CODIF measurements). Lower panel gives the same results for spacecraft 3 with compressed counting rates. 


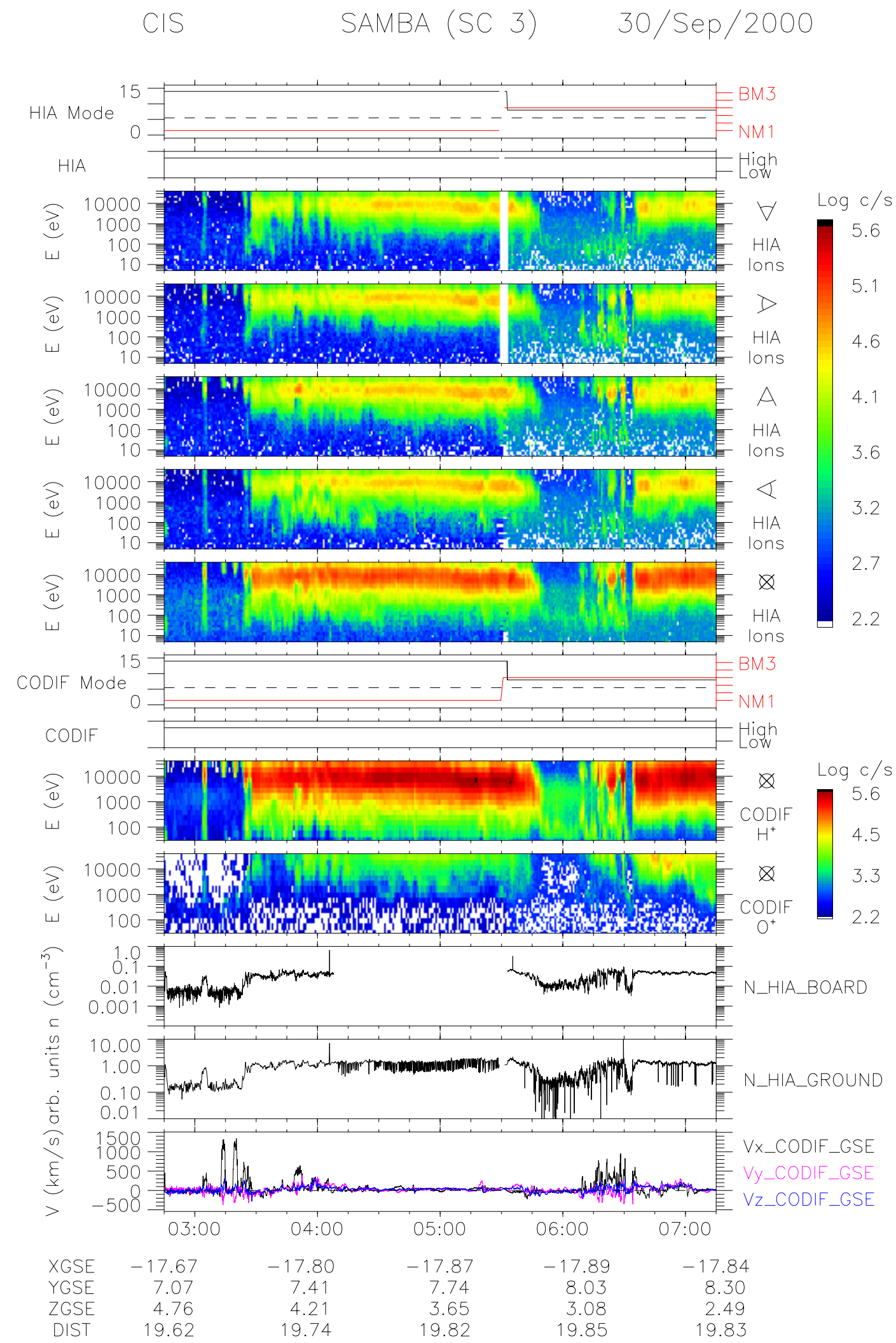

Fig. 21. CPS and near CPS measurements by the CIS experiment on 30 September 2000, between 02:45 and 07:15 UT, with spacecraft 3 . From top to bottom: HIA telemetry modes and sensitivities, energy-time spectrogram HIA, measured in the sunward, dusk, anti-sunward, dawnward looking directions and integrated over $4 \Pi$, CODIF telemetry modes and sensitivities, $\mathrm{H}^{+}$and $\mathrm{O}^{+} \mathrm{CODIF}_{\text {energy-time spec- }}$ trogram integrated over $4 \Pi$, onboard and ground calculated ion density, from HIA, and onboard GSE velocity components measured by CODIF 

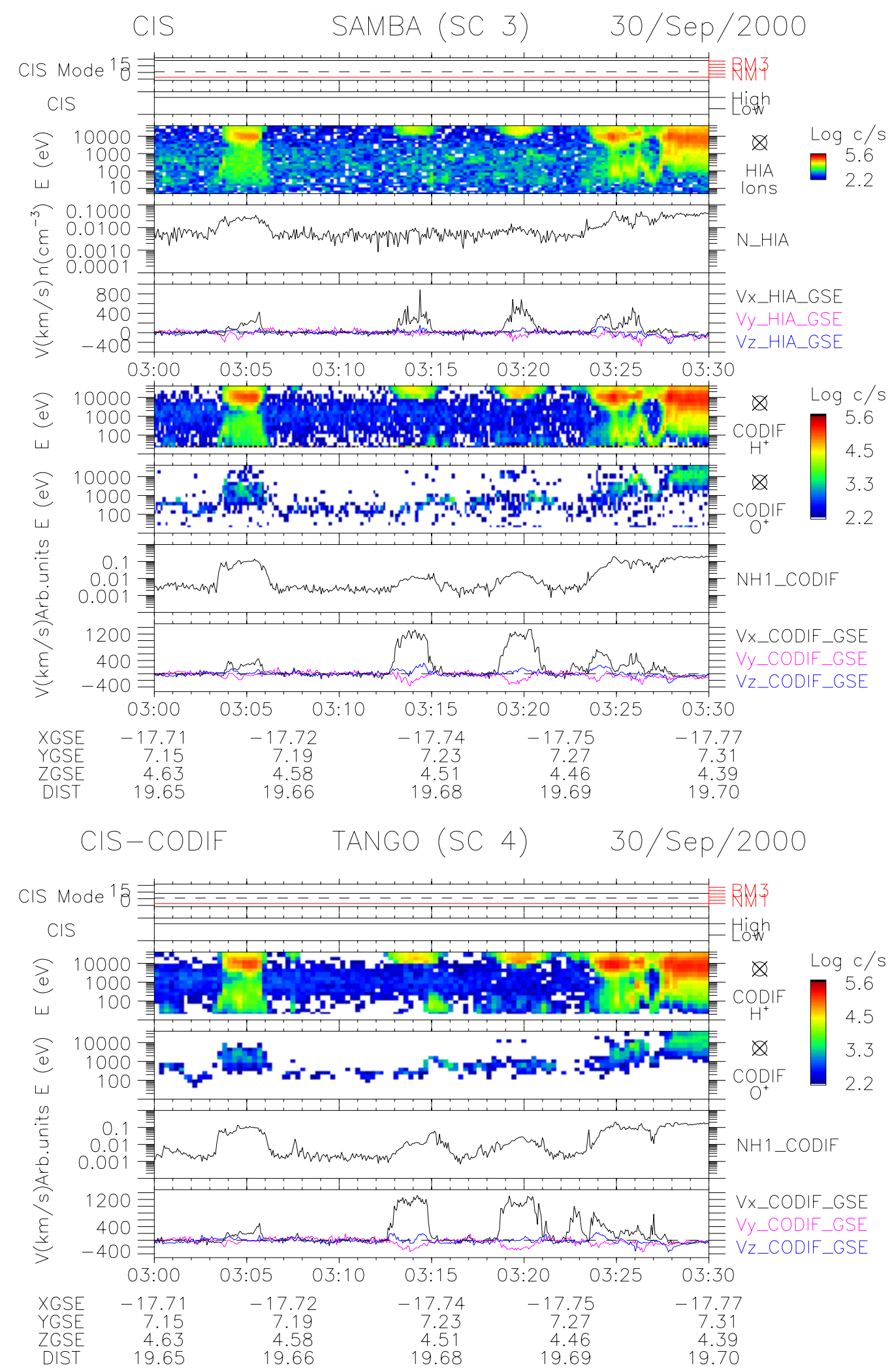

Fig. 22. Simultaneous measurements on 30 September 2000 between 03:00 and 03:30 UT with spacecraft 3 (CODIF and HIA) and spacecraft 4 (CODIF). From top to bottom, the upper panel shows for spacecraft 3, the CIS telemetry modes, the HIA energy-time spectrogram integrated over $4 \Pi$, the HIA ion density, onboard HIA GSE velocity components, $\mathrm{H}^{+}$and $\mathrm{O}^{+}$CODIF energy-time spectrogram integrated over $4 \Pi, \mathrm{H}^{+}$CODIF density and onboard GSE velocity components measured by CODIF. The lower panel shows the identical CODIF measurements for spacecraft 4 . 

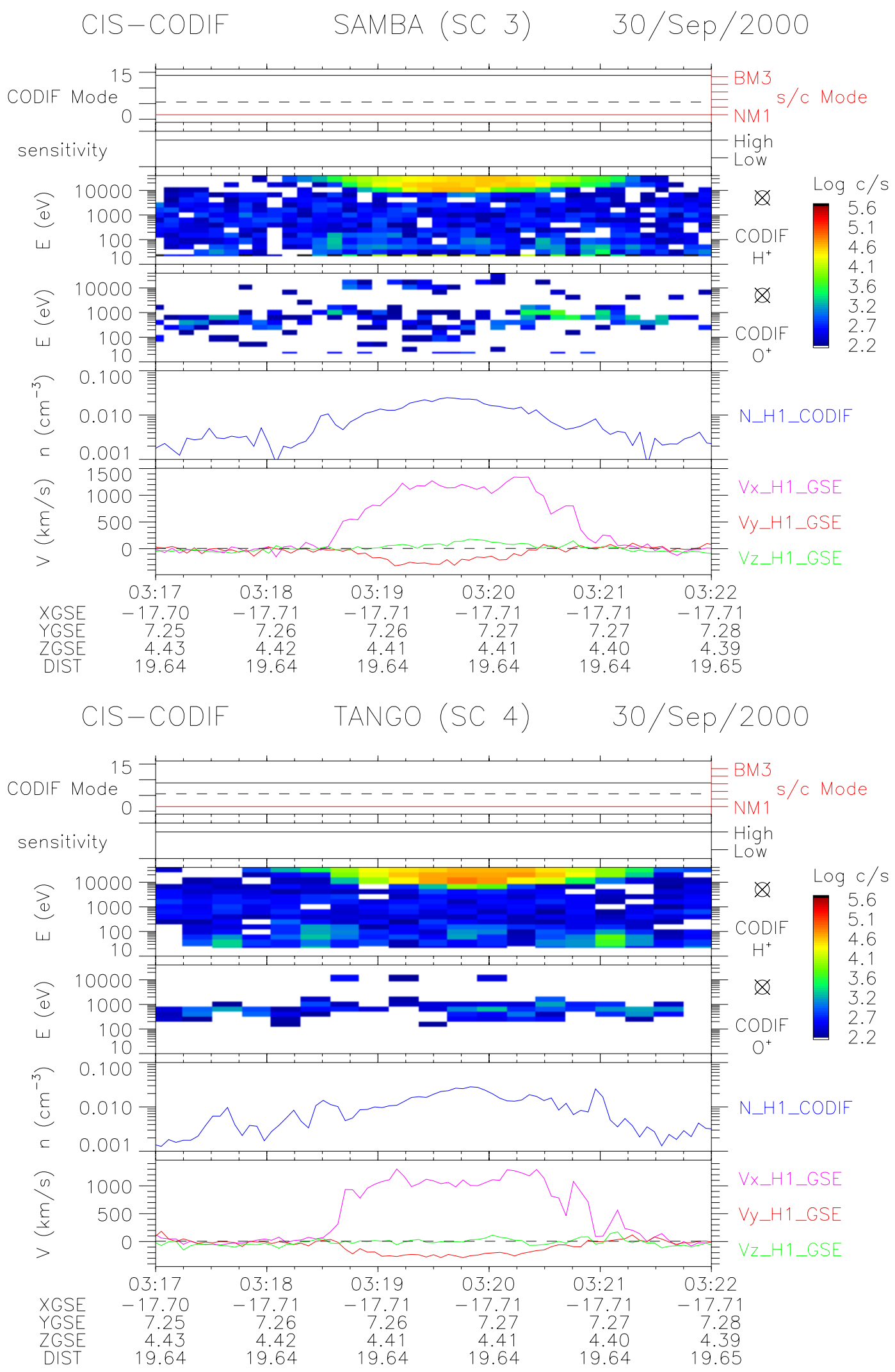

Fig. 23. Simultaneous measurements on 30 September 2000 between 03:17 and 03:22 UT with spacecraft 3 (CODIF) and spacecraft 4 (CODIF). See caption of Fig. 22 for the description of the measurements. 

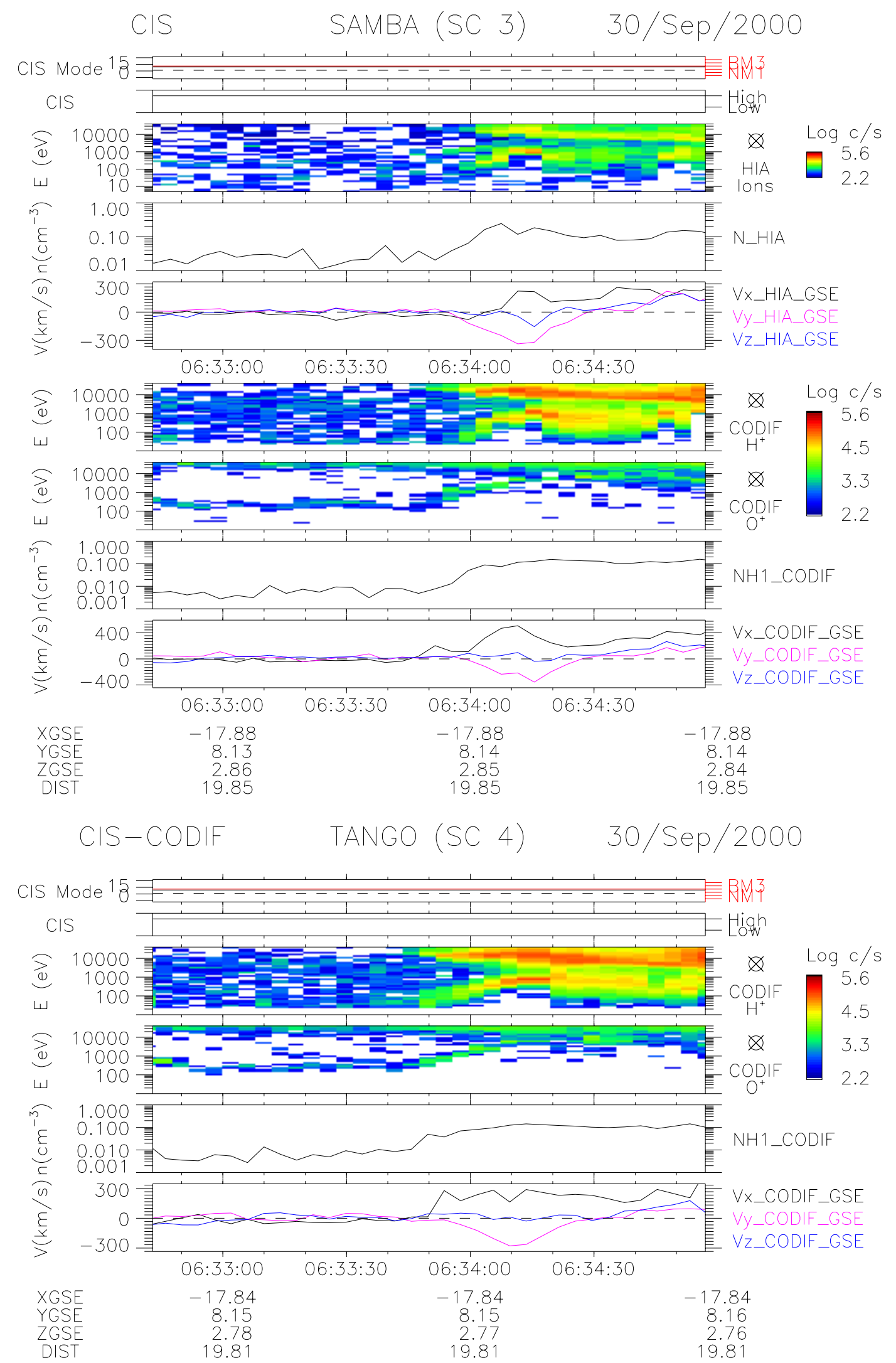

Fig. 24. Simultaneous measurements on 30 September 2000 between 06:32:15 and 06:34:55 UT with spacecraft 3 (CODIF and HIA) and spacecraft 4 (CODIF). See caption of Fig. 22 for the description of the measurements. 
than in NM1), 26762 bit/s in BM1 (normal burst mode), $6546 \mathrm{bit} / \mathrm{s}$ in BM2 (WEC/WBB TR mode) and $29456 \mathrm{bit} / \mathrm{s}$ in BM3 (event memory readout). NM1 and BM1 are the normal modes. BM3 is a special mode used only to dump the instrument's scratch memory.

\subsection{On board data-processing system}

Due to the high sensitivity and high intrinsic velocity-space resolution of the CIS instruments, continuous transmission of the complete 3D ion distributions sampled at the full time and angular resolution would require impossibly large bitrates. Therefore, extensive on board data-processing is a fundamental aspect of the CIS experiment. The CIS flight software has been designed to meet the scientific requirements of the mission even in limited transmission bit-rate allocation conditions.

First, the instrument data system (DPS) controls the operation and data collection of the two CODIF and HIA instruments. It formats the data for the telemetry channel, and receives and executes commands. In addition, the DPS analyses and compresses on board the tremendous amount of data to maximise the scientific return despite the limited CIS telemetry allocation. The DPS and the CODIF instrument are integrated in one box called CIS-1, and HIA is integrated in another box called CIS-2.

The first stage in the reduction of the CODIF data is to classify the data by species and position, and then to sum the counts in each mass/angle bin in an incrementing memory accumulator. The species determination is done by comparing the time-of-flight value of an event with a set of thresholds stored in a look-up table. There are 5 thresholds stored for each energy step, corresponding to a low threshold for $\mathrm{H}^{+}$ and $\mathrm{He}^{++}$, threshold between $\mathrm{He}^{++}$and $\mathrm{He}^{+}$, a top threshold for $\mathrm{He}^{+}$, and a low and a high threshold for $\mathrm{O}^{+}$. An example of the threshold locations over a range of energies is shown in Fig. 16. These accumulated counts are the input to both the moment calculation and to the transmitted distribution functions.

\subsubsection{Moments}

Moments of the distribution functions measured by the analysers are computed by the DPS and continuously transmitted with maximum time resolution ( 1 spin period or $4 \mathrm{~s}$ ) for CODIF (for four masses) and the HIA instruments. These moments include particle density $N_{i}$ (including partial densities over several energy ranges for CODIF, and sunward and anti-sunward densities for HIA), the three components of the flow vector $\boldsymbol{V}_{\boldsymbol{i}}$, the six unique components of the momentum flux tensor, and the ion heat flux vector. From these, the full pressure tensor can be deduced, as well as the temperature anisotropies $T_{\|} / T_{\perp}$. Full $4 \pi$ space coverage of the analysers and their clean response function guarantee a high accuracy for the onboard computed moments. To calculate moments, integrals over the distribution function are approximated by summing the products of the measured count rates with the appropriate energy/angle weighting over the sampled distribution.

In addition to instrument sensitivity and calibration, the accuracy of the computed moments is primarily affected by the finite energy and angle resolution, and by the finite energy range. The requirement of instrumental accuracy is best demonstrated in the measurements of mass flow through the magnetospheric boundary and in the computation of the current density in the current layers, such as the magnetopause and the Flux Transfer Events (FTEs). Directional errors in the bulk velocity of less than $2^{\circ}$ and relative errors less than $5 \%$ in the product of the bulk velocity times the number density of the different species are highly desirable. As for the mass flow, quantitative tests of other conservation laws (stress and energy balance) require measurements of plasma moments with uncertainties less than 5\%. Paschmann et al. (1986) tested the capability of the AMPTE/IRM plasma instrument in a simulation study. For parameters typically observed in high-speed flow events, the simulation shows that density, velocity, temperature and pressure are accurately measured to within $5 \%$. With the better azimuthal coverage and resolution of the CIS instruments, improved accuracy (in comparison to AMPTE/ IRM) of the plasma moments was expected by Martz (1993). The accuracy requirements concerning the analysis of two- and three-dimensional current structures, as well as shear and vortex flows, i.e. measurements strongly related to the four spacecraft aspect, are fulfilled by the capability of the instrument, as demonstrated by in-flight measurements.

\subsubsection{Reduced distributions}

Other reduced distributions, including pitch-angle distributions, averages (over 2 to 5 spin periods) or snapshots of the $3 \mathrm{D}$ distributions, can be computed with resolutions dependent upon the specific scientific objectives and telemetry rate. The two-dimensional pitch-angle distribution requires far less telemetry than the full distribution, thus allowing higher time resolution. Pitch-angle distributions can be transmitted when the magnetic field direction (provided by the onboard magnetometer) is in the field of view of the detector.

\subsubsection{Onboard processing unit}

These computations in real time are a heavy processing burden, and require a sophisticated data system, both in terms of hardware and software. The data system is based on a set of two microprocessors. The main processor, located in the CIS-1 box, interfaces with the spacecraft's On-Board Data Handling System (OBDH), the magnetometer, the plasma wave experiments (DWP), and the CIS-2 processor. It is in charge of formatting telemetry data, receiving and executing commands or passing them to the other processor, and controlling the burst memory. It also controls, collects and analyses data from the CODIF. The second processor is included in the CIS-2 box and controls, collects and analyses data from 
CIS-CODIF SAMBA (SC 3) 30/Sep/2000 03:04:31.044
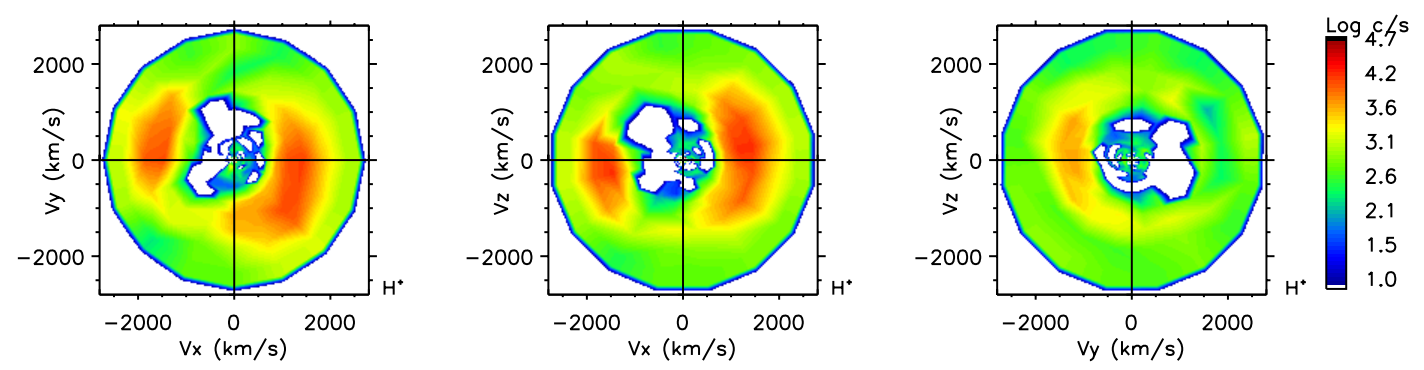

3 spin accumulation

Fig. 25. CODIF $\mathrm{H}^{+}$distribution function (accumulation over 3 spins) in GSE coordinates on spacecraft 3 on 30 September 2000 at 03:04:31 UT.
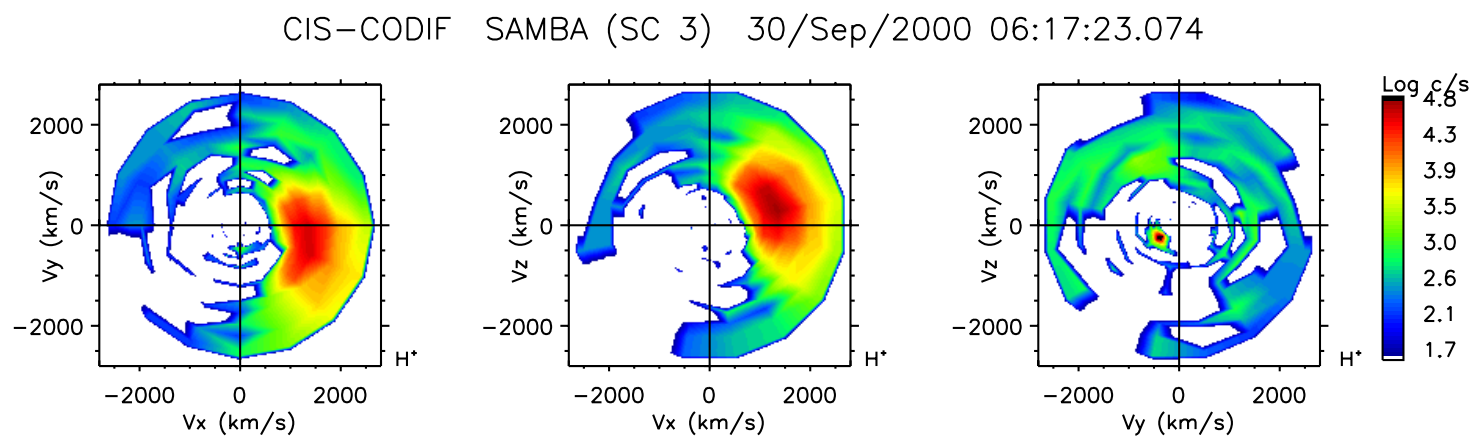

3 spin accumulation

Fig. 26. CODIF $\mathrm{H}^{+}$distribution function (accumulation over 3 spins) in GSE coordinates on spacecraft 3 on 30 September 2000 at 06:17:23 UT.

the HIA. The main processor is interfaced with the second one by a serial data line; the HIA processor compresses the data so that the serial link can transmit at the highest data rates.

\subsubsection{Scratch memory}

The CIS experiment acquires data at nearly the fastest useful rate. In order to store a series of many two- and threedimensional distributions at full time resolution, a 1 Mbyte memory is included in the instrument, so that discontinuities can be studied in detail. This scratch memory is read when the spacecraft is in BM3 telemetry mode, or in NM1 mode 15 (Table 7) when the appropriate flag is set in the software.

\subsection{Telemetry}

\subsubsection{Data products}

Tables 5 and 6 give HIA and CODIF scientific telemetry products, respectively. Products consist of onboard computed moments, one-, two- and three-dimenstional distributions and pitch-angle distributions. The high flexibility in selecting data products to be transmitted at a given period de- pends upon the telemetry mode, the bit-rate sharing between CIS-1 and 2, and, of course, the plasma environment; energy, angle, and time resolutions can be optimised to extract maximum information relevant to the scientific objectives. Data format changes are programmed within the instrument and do not require any reformatting of the spacecraft or ground data systems.

For example, HIA typically produces a data volume of 32 polar sectors times 62 energies times 32 azimuth sectors, with 16 bit-words, sampled in one spin period (4s). Such a very high data rate has to be handled by a real time operating system in order to elaborate and compress data into a few kbit $\mathrm{s}^{-1}$ telemetry stream output. All information is transmitted as log-compressed 8-bit words, except the moments that are transmitted with 12-bits. Pitch-angle distributions are instantaneous measurements when $\boldsymbol{B}$ is in the field of view of the instruments, and typical full 3D distributions are reduced to $88 \Omega$ (solid angles) by taking into account the oversampling in the polar regions.

Basically, for HIA, the high-sensitivity section has full $180^{\circ}$ coverage and hot population data are computed using data from this section. When there is a cold population, such as the solar wind, data products are provided by the small 
Table 5. HIA scientific telemetry products

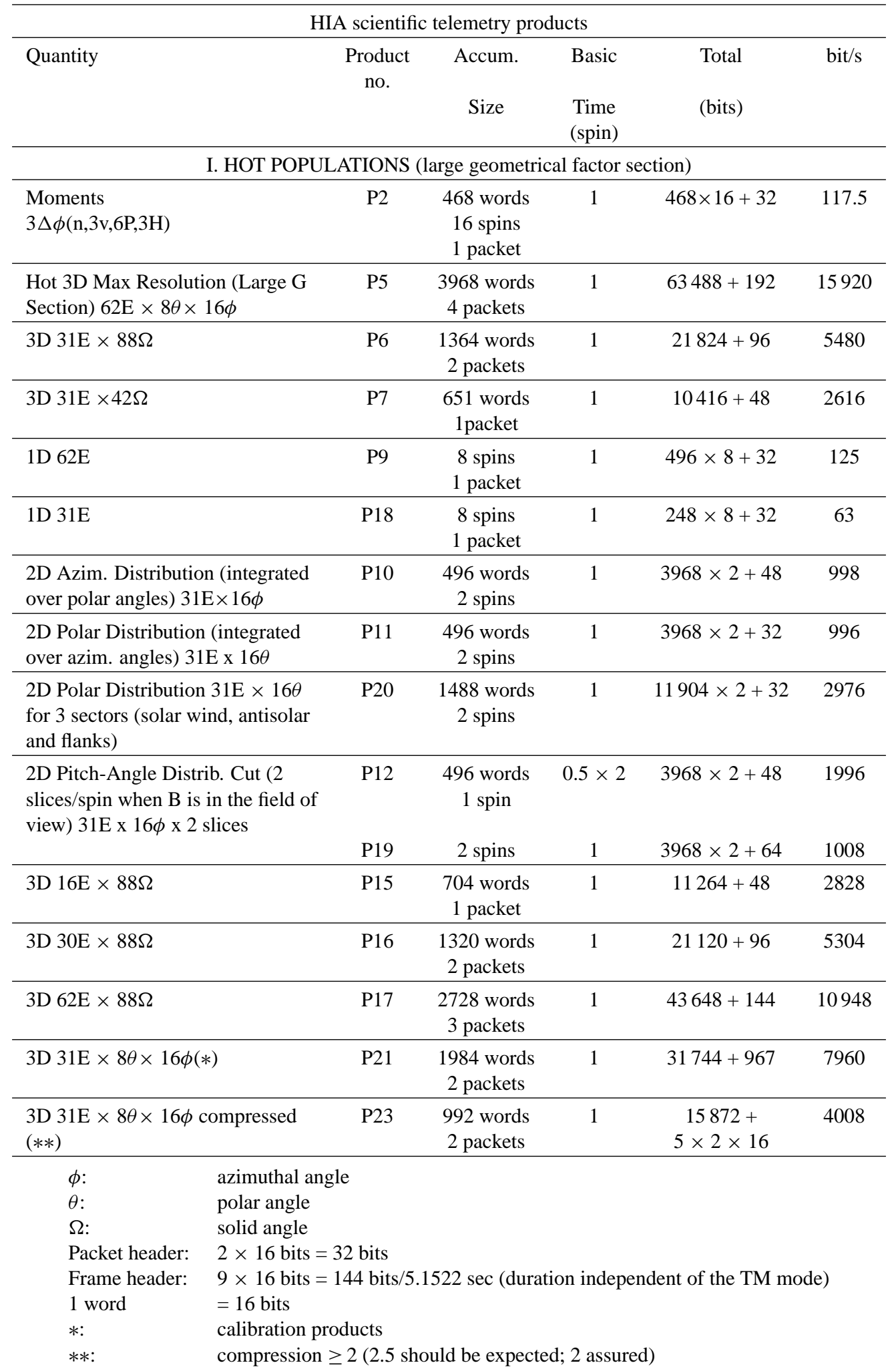

geometrical factor, but the rest of the spin $\left(360^{\circ}-45^{\circ}\right)$ is not ignored; data are taken and transmitted. Data from the large geometric factor section are also taken and transmitted.
For CODIF, $4 \mathrm{M}$ stands for the four major species: $\mathrm{H}^{+}$, $\mathrm{He}^{++}, \mathrm{O}^{+}$and $\mathrm{He}^{+}$. Sixty-four M 3D distributions can be read out at a slow rate. They give more detailed information 
Table 5. (continued)

\begin{tabular}{|c|c|c|c|c|c|}
\hline \multicolumn{6}{|c|}{ HIA scientific telemetry products } \\
\hline \multirow[t]{2}{*}{ Quantity } & \multirow{2}{*}{$\begin{array}{l}\text { Product } \\
\text { no. }\end{array}$} & Accum. & Basic & Total & \multirow[t]{2}{*}{$\mathrm{bit} / \mathrm{s}$} \\
\hline & & Size & $\begin{array}{l}\text { Time } \\
(\text { spin })\end{array}$ & (bits) & \\
\hline \multicolumn{6}{|c|}{ II. COLD POPULATIONS - solar wind (small geometrical factor section) } \\
\hline $\begin{array}{l}\text { Cold moments for solar wind } \\
2 \Delta \mathrm{E}(\mathrm{n}, 3 \mathrm{v}, 6 \mathrm{P}, 3 \mathrm{H})\end{array}$ & P4 & $\begin{array}{l}156 \text { words } \\
16 \text { spins }\end{array}$ & 1 & $\begin{array}{c}312 \times 16+ \\
16 \times 18\end{array}$ & 82.5 \\
\hline $3 \mathrm{D} 31 \mathrm{E} \times 8 \theta \times 8 \phi($ cold $3 \mathrm{D})$ & P8 & $\begin{array}{l}992 \text { words } \\
1 \text { packet }\end{array}$ & 1 & $15872+32$ & 3976 \\
\hline $\begin{array}{l}\text { 2D Cold Azim. Distrib. } \\
(\theta \text { integration }) 31 \mathrm{E} \times 8 \phi\left(5.6^{\circ} \text { each }\right)\end{array}$ & $\mathrm{P} 13$ & $\begin{array}{l}496 \text { words } \\
4 \text { spins }\end{array}$ & 1 & $1984 \times 4+32$ & 498 \\
\hline $\begin{array}{l}\text { 2D Cold Polar Distrib. } \\
(\phi \text { integration }) 31 \mathrm{E} \times 8 \theta\left(5.6^{\circ} \text { each }\right)\end{array}$ & P14 & $\begin{array}{l}496 \text { words } \\
4 \text { spins }\end{array}$ & 1 & $1984 \times 4+32$ & 498 \\
\hline $3 \mathrm{D} 31 \mathrm{E} \times 8 \theta \times 16 \phi(*)$ & $\mathrm{P} 22$ & $\begin{array}{l}1984 \text { words } \\
2 \text { packets }\end{array}$ & 1 & $31744+32$ & 7944 \\
\hline $\begin{array}{l}3 \mathrm{D} 31 \mathrm{E} \times 8 \theta \times 16 \phi \text { compressed } \\
(* *)\end{array}$ & $\mathrm{P} 24$ & $\begin{array}{l}992 \text { words } \\
1 \text { packet }\end{array}$ & 1 & $15872+4 \times 16$ & 3984 \\
\hline \multicolumn{6}{|c|}{ III. PROM PRODUCTS } \\
\hline PROM HIA Sweep Diagnostics & $\mathrm{P} 0$ & 1 & 1 & $1024+32$ & 264 \\
\hline PROM HIA 48C, 32E & $\mathrm{P} 1$ & 1 & 1 & $12288+32$ & 3080 \\
\hline \multicolumn{6}{|c|}{ IV. VARIOUS PRODUCTS } \\
\hline HIA Memory I/O Read & P61 & 1 & - & $32+32$ & 16 \\
\hline HIA Memory Dump & P62 & 1 & - & $4112+32$ & 1036 \\
\hline
\end{tabular}

about the presence of minor species. Four M, $88 \Omega$ (solid angles), 3D distributions should be read out as often as possible, after all the other data types have been accommodated. A priority scheme for the time resolution is given according to the abundance of the species: $\mathrm{H}^{+}$with the highest resolution, $\mathrm{He}^{++}$or $\mathrm{O}^{+}$with the highest resolution or slower by a factor of $2 \mathrm{He}^{+}$or other species with the factor of 2 or factor 4 slower.

\subsubsection{Data compression}

A linear compression scheme is implemented as part of the onboard CIS software, which allows the possibility to transmit compressed 3D distributions more often. The compression factor can be adjusted by setting new values to the compression parameters. A number of simulations have proven that a factor of 2 in the compression factor can easily be reached without any loss of data. The chosen algorithm for this compression is based on the evaluation of the dispersion of the maximum of a Data Block around the average of the 8 successive value data blocks themselves. If the maximum (Max) satisfies the following:

$\operatorname{Max}-k^{*} \sqrt{(\operatorname{Max})}<$ Data Block Average $_{\text {, }}$

where $k$ is an ajustable parameter factor used to set the dispersion, the data are assumed to be equal to the Data Block $k_{\text {Average }}$ which is transmitted as representative of the whole Data Block. Otherwise the Data Block length is divided by a factor of 2 and the above inequality is applied until the relation is satisfied or the Data Block length has been reduced to 1 . If $k$ is assumed to be 0 , then the compression becomes error-free.

\subsubsection{Remote-sensing distribution with CODIF}

Close to the boundaries a distribution of four angles at $90^{\circ}$ pitch-angle (phase $0^{\circ}, 90^{\circ}, 180^{\circ}, 270^{\circ}$ ) is accumulated for two species $\left(\mathrm{H}^{+}\right.$and $\left.\mathrm{O}^{+}\right)$in the four highest energies, by making use of the distinct gradient anisotropies of these ions within about one gyroradius of the boundary. This allows the boundary motions to be traced. Since no automatic sensing of the boundaries is implemented on Cluster, this data product is included in the telemetry when the satellites are close to the nominal position of the interesting boundaries. Generally, data from the High Side section of CODIF are used, which provides substantial counting statistics at all magnetospheric boundaries. The accuracy of this analysis will be tested using the full 3D distributions during the time periods when they are available with the full time resolution in Burst Mode.

\subsubsection{CODIF live pulse height data}

For each particle, CODIF measures the following parameters: 
Table 6. CODIF scientific telemetry products

\begin{tabular}{|c|c|c|c|c|c|}
\hline \multicolumn{6}{|c|}{ CODIF scientific telemetry products } \\
\hline Quantity & Product no. & $\begin{array}{c}\text { Packet } \\
\text { number } \\
\text { s }\end{array}$ & $\begin{array}{c}\text { Basic } \\
\text { Time } \\
\text { (spins) }\end{array}$ & $\begin{array}{l}\text { Total } \\
\text { bits }\end{array}$ & $\mathrm{bit} / \mathrm{s}$ \\
\hline \multicolumn{6}{|c|}{ I. HOT POPULATIONS } \\
\hline $\begin{array}{l}\text { Moments } \\
3 \Delta \mathrm{E}(\mathrm{n}, 3 \mathrm{v}, 6 \mathrm{P}, 3 \mathrm{H}) \times 4 \mathrm{M}\end{array}$ & $\mathrm{P} 7$ & 1 & 1 & $1872+32$ & 476 \\
\hline $\begin{array}{l}3 \mathrm{D} 64 \mathrm{M} \times 8 \mathrm{E} \times 6 \Omega \\
(6 \Omega: 2 \text { polar, } 4 \text { perpendicular })\end{array}$ & $\mathrm{P} 11$ & 2 & 2 & $24576+64$ & 3080 \\
\hline $3 \mathrm{D}$ protons $1 \mathrm{M} \times 16 \mathrm{E} \times 88 \Omega$ & $\mathrm{P} 12$ & 1 & 1 & $11264+32$ & 2824 \\
\hline $3 \mathrm{D}$ protons $1 \mathrm{M} \times 31 \mathrm{E} \times 88 \Omega$ & $\mathrm{P} 13$ & 2 & 1 & $21824+64$ & 5472 \\
\hline $3 \mathrm{D}$ protons $1 \mathrm{M} \times 31 \mathrm{E} \times 24 \Omega$ & $\mathrm{P} 14$ & 1 & 1 & $5952+32$ & 1496 \\
\hline $3 \mathrm{D} \mathrm{He}^{++} 1 \mathrm{M} \times 16 \mathrm{E} \times 88 \Omega$ & $\mathrm{P} 15$ & 1 & 1 & $11264+32$ & 2824 \\
\hline $3 \mathrm{D} \mathrm{He}^{++} 1 \mathrm{M} \times 31 \mathrm{E} \times 88 \Omega$ & $\mathrm{P} 16$ & 2 & 1 & $21824+64$ & 5472 \\
\hline $3 \mathrm{D} \mathrm{He}^{+}, \mathrm{O}^{+} 2 \mathrm{M} \times 16 \mathrm{E} \times 88 \Omega$ & $\mathrm{P} 17$ & 2 & 1 & $22528+64$ & 5648 \\
\hline $3 \mathrm{D} \mathrm{He}^{+}, \mathrm{O}^{+} 2 \mathrm{M} \times 31 \mathrm{E} \times 88 \Omega$ & $\mathrm{P} 18$ & 4 & 1 & $43776+128$ & 10944 \\
\hline $3 \mathrm{D} \mathrm{He}^{+} 1 \mathrm{M} \times 16 \mathrm{E} \times 88 \Omega$ & $\mathrm{P} 32$ & 1 & 1 & $11264+32$ & 2824 \\
\hline $3 \mathrm{D} \mathrm{O}^{+} 1 \mathrm{M} \times 16 \mathrm{E} \times 88 \Omega$ & $\mathrm{P} 33$ & 1 & 1 & $11264+32$ & 2824 \\
\hline $3 \mathrm{D} \mathrm{He}^{+} 1 \mathrm{M} \times 31 \mathrm{E} \times 88 \Omega$ & $\mathrm{P} 34$ & 2 & 1 & $21824+64$ & 5472 \\
\hline $3 \mathrm{D} \mathrm{O}^{+} 1 \mathrm{M} \times 31 \mathrm{E} \times 88 \Omega$ & P35 & 2 & 1 & $21824+64$ & 5472 \\
\hline $2 \mathrm{D} 4 \mathrm{M} \times 31 \mathrm{E} \times 16 \phi^{*}$ & P19 & 1 & 1 & $15872+32$ & 3976 \\
\hline $2 \mathrm{D} 2 \mathrm{M} \times 16 \mathrm{E} \times 16 \phi\left(\right.$ protons $\left.+\mathrm{He}^{++}\right) * *$ & $\mathrm{P} 21$ & 1 & 1 & $4096+32$ & 1032 \\
\hline or $4 \mathrm{M} \times 16 \mathrm{E} \times 8 \phi * *$ & $\mathrm{P} 20$ & 1 & 1 & $4096+32$ & 1032 \\
\hline $2 \mathrm{D}$ protons $1 \mathrm{M} \times 31 \mathrm{E} \times 32 \phi$ & $\mathrm{P} 22$ & 1 & 1 & $7936+32$ & 1992 \\
\hline $\begin{array}{l}2 \mathrm{D} \text { PAD Cut } 4 \mathrm{M} \times 16 \mathrm{E} \times 8 \theta \\
(2 \text { slices/spin when } \mathrm{B} \text { is in the field of view }) * *\end{array}$ & $\mathrm{P} 23$ & $1 /$ slice & 0.5 & $\begin{array}{l}4096 \times 2 \\
+2 \times 32 \\
\end{array}$ & $\begin{array}{c}2064 \\
(1032 / \text { slice }) \\
\end{array}$ \\
\hline $\begin{array}{l}2 \mathrm{D} \text { PAD Cut } 4 \mathrm{M} \times 31 \mathrm{E} \times 8 \theta \\
(2 \text { slices/spin when } \mathrm{B} \text { is in the field of view }) *\end{array}$ & $\mathrm{P} 24$ & $1 /$ slice & 0.5 & $\begin{array}{l}7936 \times 2 \\
+2 \times 32 \\
\end{array}$ & 3984 \\
\hline $\begin{array}{l}\text { Monitor Counting Rates } \\
18 \text { signals } \times 16 \mathrm{E} \times 16 \phi\end{array}$ & $\mathrm{P} 27$ & 8 & 32 spins & $36864+256$ & 290 \\
\hline $\begin{array}{l}\text { Live Pulse Height Data (selected events) } \\
\text { Time of flight: } 8 \text { bits } \\
\text { Azim. Position: } 5 \text { bits } \\
\text { Energy Step: } 7 \text { bits } \\
\text { Sector: } 3 \text { bits } \\
\text { Proton mode: } 1 \text { bit }\end{array}$ & $\mathrm{P} 28$ & 1 & every 2 spins & $\begin{array}{c}24 \times k+32 \\
k>1\end{array}$ & $\begin{array}{l}\text { Depending } \\
\text { of } k \text { value }\end{array}$ \\
\hline \multicolumn{6}{|c|}{ II. RPA MODES } \\
\hline $\begin{array}{l}\text { RPA diagnostic product } \\
3 \mathrm{D} 4 \mathrm{M} \times 16 \mathrm{E} \times 88 \Omega\end{array}$ & P29 & 4 & $1 \mathrm{spin}$ & $45056+256$ & 11328 \\
\hline \multicolumn{6}{|c|}{ III. COLD POPULATIONS } \\
\hline $\begin{array}{l}\text { Cold Populations Moments } \\
3 \Delta \mathrm{E}(\mathrm{n}, 3 \mathrm{v}, 6 \mathrm{P}, 3 \mathrm{H}) \times 4 \mathrm{M}\end{array}$ & $\mathrm{P} 9$ & 1 & 1 & $1872+32$ & 476 \\
\hline
\end{tabular}

Time-of-flight: $\quad 8$ bits

Azimuthal position: 5 bits

Proton on/off: $\quad 1$ bit

Energy step: $\quad 7$ bits

Pixel number: $\quad 3$ bits

Total: (giving 256 values)

(32 sectors)

(one between 128 elementary steps)
5.2.5 CODIF monitor rates

To check the performance and the counting efficiency of CODIF, certain monitor rates have to be accumulated and transmitted with the science data: 
Table 6. (continued)

\begin{tabular}{|c|c|c|c|c|c|c|}
\hline \multicolumn{7}{|c|}{ CODIF scientific telemetry products } \\
\hline Quantity & & Product no. & $\begin{array}{c}\text { Packet } \\
\text { number } \\
\text { s }\end{array}$ & $\begin{array}{l}\text { Basic } \\
\text { Time } \\
\text { (spins) }\end{array}$ & $\begin{array}{l}\text { Total } \\
\text { BITS }\end{array}$ & $\begin{array}{l}\text { (bits) } \\
\text { bit/s }\end{array}$ \\
\hline \multicolumn{7}{|c|}{ IV. COMPRESSED PRODUCTS } \\
\hline $3 \mathrm{D} \mathrm{H}^{+} 1$ & $\mathrm{M} \times 16 \mathrm{E} \times 88 \Omega$ & P39 & 1 & 1 & - & - \\
\hline $3 \mathrm{D} \mathrm{H}^{+} 1$ & $\mathrm{M} \times 31 \mathrm{E} \times 88 \Omega$ & $\mathrm{P} 40$ & 2 & 1 & - & - \\
\hline $3 \mathrm{D} \mathrm{H}^{+} 1$ & $\mathrm{M} \times 31 \mathrm{E} \times 24 \Omega$ & $\mathrm{P} 41$ & 1 & 1 & - & - \\
\hline $3 \mathrm{D} \mathrm{He}{ }^{+-}$ & $1 \mathrm{M} \times 16 \mathrm{E} \times 88 \Omega$ & $\mathrm{P} 42$ & 1 & 1 & - & - \\
\hline $3 \mathrm{D} \mathrm{He}^{+-}$ & $1 \mathrm{M} \times 31 \mathrm{E} \times 88 \Omega$ & $\mathrm{P} 43$ & 2 & 1 & - & - \\
\hline $3 \mathrm{D} \mathrm{He}^{+} /$ & $\mathrm{O}^{+} 2 \mathrm{M} \times 16 \mathrm{E} \times 88 \Omega$ & $\mathrm{P} 44$ & 2 & 1 & - & - \\
\hline $3 \mathrm{D} \mathrm{He}^{+} /$ & $\mathrm{O}^{+} 2 \mathrm{M} \times 31 \mathrm{E} \times 88 \Omega$ & $\mathrm{P} 45$ & 4 & 1 & - & - \\
\hline $3 \mathrm{D} \mathrm{He}+$ & $1 \mathrm{M} \times 16 \mathrm{E} \times 88 \Omega$ & $\mathrm{P} 46$ & 1 & 1 & - & - \\
\hline $3 \mathrm{D} \mathrm{O}^{+} 1$ & $\mathrm{M} \times 16 \mathrm{E} \times 88 \Omega$ & $\mathrm{P} 47$ & 1 & 1 & - & - \\
\hline $3 \mathrm{D} \mathrm{He}+$ & $1 \mathrm{M} \times 31 \mathrm{E} \times 88 \Omega$ & $\mathrm{P} 48$ & 2 & 1 & - & - \\
\hline $3 \mathrm{D} \mathrm{O}^{+} 1$ & $\mathrm{M} \times 31 \mathrm{E} \times 88 \Omega$ & $\mathrm{P} 49$ & 2 & 1 & - & - \\
\hline \multicolumn{7}{|c|}{ V. BURST PRODUCTS } \\
\hline Burst Me & mory Counters & P36 & 16 & 1 & $\begin{array}{c}(16384+32) \\
\times 16\end{array}$ & 65664 \\
\hline Burst Sta & & P37 & 1 & 1 & $448+32$ & 120 \\
\hline Burst Tri & gger & P38 & 1 & - & $2912+32$ & 736 \\
\hline \multicolumn{7}{|c|}{$\begin{array}{l}\text { VI. VARIOUS PRODUCTS } \\
\end{array}$} \\
\hline PROM p & oduct. Sweep Diagnostics & $\mathrm{P} 0$ & 1 & 1 & $4096+32$ & 1032 \\
\hline PROM p & oduct. 48C, 32E & $\mathrm{P} 1$ & 1 & 1 & $12288+32$ & 3080 \\
\hline PROM p & oduct. $2 \times 18 \mathrm{C}, 32 \mathrm{E}$ & $\mathrm{P} 2$ & 1 & 1 & $9216+32$ & 2312 \\
\hline $\mathrm{PROM} / \mathrm{B}$ & EPROM product. Raw events & P3 & 1 & 1 & $16384+32$ & 4104 \\
\hline PROM p & oduct. Full events & $\mathrm{P} 4$ & 1 & 1 & $16384+32$ & 4104 \\
\hline PROM/E & EPROM prod. WEC IEL data & P5 & 1 & 1 & $512+32$ & 136 \\
\hline $\mathrm{PROM} / \mathrm{E}$ & EPROM prod. FGM IEL data & P6 & 1 & 1 & $4096+32$ & 1032 \\
\hline Memory & I/O Read & P61 & 1 & - & $32+32$ & 16 \\
\hline Memory & Dump & P62 & 1 & - & $4112+32$ & 1036 \\
\hline \multicolumn{7}{|c|}{ azimuthal angle (spin phase angle) } \\
\hline \multicolumn{7}{|c|}{ polar angle } \\
\hline \multicolumn{7}{|c|}{ solid angle } \\
\hline \multicolumn{7}{|c|}{ near $90^{\circ}$ pitch-angles (the highest possible angle) for gyrotropic distributions (for 4 high energies) } \\
\hline \multicolumn{7}{|c|}{ best possibility } \\
\hline \multicolumn{7}{|c|}{ basic use } \\
\hline \multirow{2}{*}{\multicolumn{7}{|c|}{$\begin{array}{l}\text { possibility to have different time of resolution for the different masses } \\
\text { protons, } \mathrm{He}^{+}, \mathrm{He}^{++}, \mathrm{O}^{+}\end{array}$}} \\
\hline & & & & & & \\
\hline \multicolumn{7}{|c|}{32 bits } \\
\hline
\end{tabular}

2 Starts

2 Coincidences

16 Start positions

4 Stop positions

To cut down on the bit-rate, a specific scheme is used by which only every fourth energy step and every eighth sector are transmitted at a time. A cycle is completed after 32 spins.

\subsubsection{Telemetry formats}

Instrument science and housekeeping data are read over a single serial interface; the two types are differentiated by separate word gates. Telemetry is collected as a series of blocks, representing a fixed number per telemetry frame. The telemetry frames are always $5.152222 \mathrm{~s}$ in duration, independent of the telemetry mode, and are synchronised by a "Re- 
set" pulse that occurs at the beginning of each frame. Housekeeping data consists of 54 bytes per telemetry frame. Science can be collected in a variety of modes with different bitrates; these modes are subdivided into "Normal" and "Burst" Modes, differentiated by the number of blocks per frame (10 for normal and 62 for burst). The different bit-rates for Normal Mode are generated by changing the number of words per block. BM3 is a special mode used only to dump the instrument's scratch memory; it is not an ordinary operating mode. Two contingency modes exist in which all available data go either to CIS-1 (CODIF) or to CIS-2 (HIA).

The four Cluster spacecraft fly through a number of different plasma environments, and there must be a mechanism to change the mode of the instrument with a minimum number of commands when moving from one region to another. The CIS instruments have a large amount of flexibility either in the selection of the operating mode or in the reduction of the data necessary to fit the available telemetry bandwith. The instrument must be capable of making many changes to the operational details in response to a few commands.

Table 7 shows the 16 CIS basic operation modes with the bit-rate sharing between CODIF and HIA, defined for each spacecraft bit-rate mode. The CIS instruments operate in the different regions of the Earth's environment in these 16 operative modes. For the five telemetry regimes foreseen (forgetting the HK and BM3 modes), this gives a total amount of 80 science data transmission schemes. Each basic scheme corresponds to a given sequence of products, spanning from the moments of the ion distributions to the 3D.

Roughly speaking, all 16 operative regimes can be grouped into solar wind tracking oriented modes, solar wind study modes, with the priority on the backstreaming ions, magnetospheric modes, an RPA mode and a calibration mode. Moreover, part of these solar-wind and magnetospheric modes are duplicated in a similar mode in which $3 \mathrm{D}$ compression is introduced (modes 4, 5, 13, and 14).

For HIA, the 16 basic CIS operation modes have also been implemented, mixing basic products defined in Table 5. These 16 modes can be grouped into 2 mode families, according to the plasma populations encountered along the Cluster orbit: the so-called (a) "magnetospheric" modes, and (b) "solar wind" modes. In both modes, moments are systematically transmitted, and computed every spin from the data acquired on the high-sensitive half-hemisphere ("high $G$ " section) when the spacecraft are inside the magnetosphere, and from the attenuated half-hemisphere section ("low $g$ ") when the spacecraft are in the interplanetary medium. In this way one of the goals of the mission, i.e. to be able to produce high-resolution $(4 \mathrm{~s})$ moments by onboard computation, has been fullfilled for all the listed regimes apart from the calibration mode. The computed moments can be used onboard to drive automatic operative mode changes (when this option has been remotely enabled) to better follow fluctuations that require fast sensitivity-adapting capabilities or to select the best energy sweep regime to cover the local solar wind distribution. This energy tracing in the solar wind has been successfully tested. The automatic mode change from mag- netospheric to solar wind modes and vice versa remains to be tested.

"Magnetosphere" basic modes stay relatively simple, i.e. the full energy-angle ranges are systematically covered, and the different data products (including moments) are deduced from the $62 \mathrm{E} \times 88 \Omega$ energy solid angle count rate matrices accumulated on the "high $G$ " section.

"Solar wind" modes allow for a precise and fast measurement $(4 \mathrm{~s})$ of the ion flow parameters $\left(\mathrm{H}^{+}, \mathrm{He}^{++}\right)$. For that to occur in the solar wind, the sweep energy range is automatically reduced and adapted for every spin, centred on the main solar wind velocity by using a criterion based on the $\mathrm{H}^{+}$thermal and bulk velocities computed during the previous spin. Moreover, detailed 3D distributions (e.g. for upstreaming ions and/or for interplanetary disturbances) are included in the basic products transmitted to the telemetry.

In both regions, and within the HIA telemetry allocation, a maximum bit-rate has been allowed for the transmission as often as possible of full size (or reduced) 3D distributions.

Science data packets include a number of data products from both HIA and CODIF in a flexible format. Data are time-tagged in such a way as to allow for absolute timing of the data on the ground. The format allows the bit-rate allocations of the various data products to be changed relatively easily with minimal impact on ground processing. All auxiliary data necessary to analyse the data, such as instrument operational mode and timing information, are included in science data products, since it could be difficult to recombine housekeeping packets with the science packets.

Finally, housekeeping data (81 bit $\mathrm{s}^{-1}$ ), extensively used during spacecraft development tests, give all the information needed to follow the health and safety of the instrument. Table 8 shows the scientific products of HIA transmitted nominally in the various telemetry modes.

\subsection{Processing unit}

One of the decisive variables which affects the instrument operation is the telemetry mode; when the telemetry mode changes, the CIS instrument receives a single command and changes accordingly its bit-rate allocation and data product collection mechanism to match the available telemetry. Some instrument parameters stay mode-independent and are programmable, such as MCP voltage.

The DPS consists of a small PROM, some EEPROM, and some RAM memories. The non-volatile EEPROM memory contains most of the onboard code and parameter tables, while the RAM memory is used primarily to hold data blocks and some operational parameters, and the PROM memory contains the bootstrap code needed to load or change the EEPROM. The EEPROM memory cannot be read while it is being programmed, and programming takes several millisec per block; it contains most of the operational parameters so that they do not have to be reloaded on power-up.

As a basic philosophy, the default operational parameters are kept in EEPROM memory, while the current operational parameters are in RAM memory. The telemetry mode inde- 
Table 7. Cluster-2 CIS operations modes

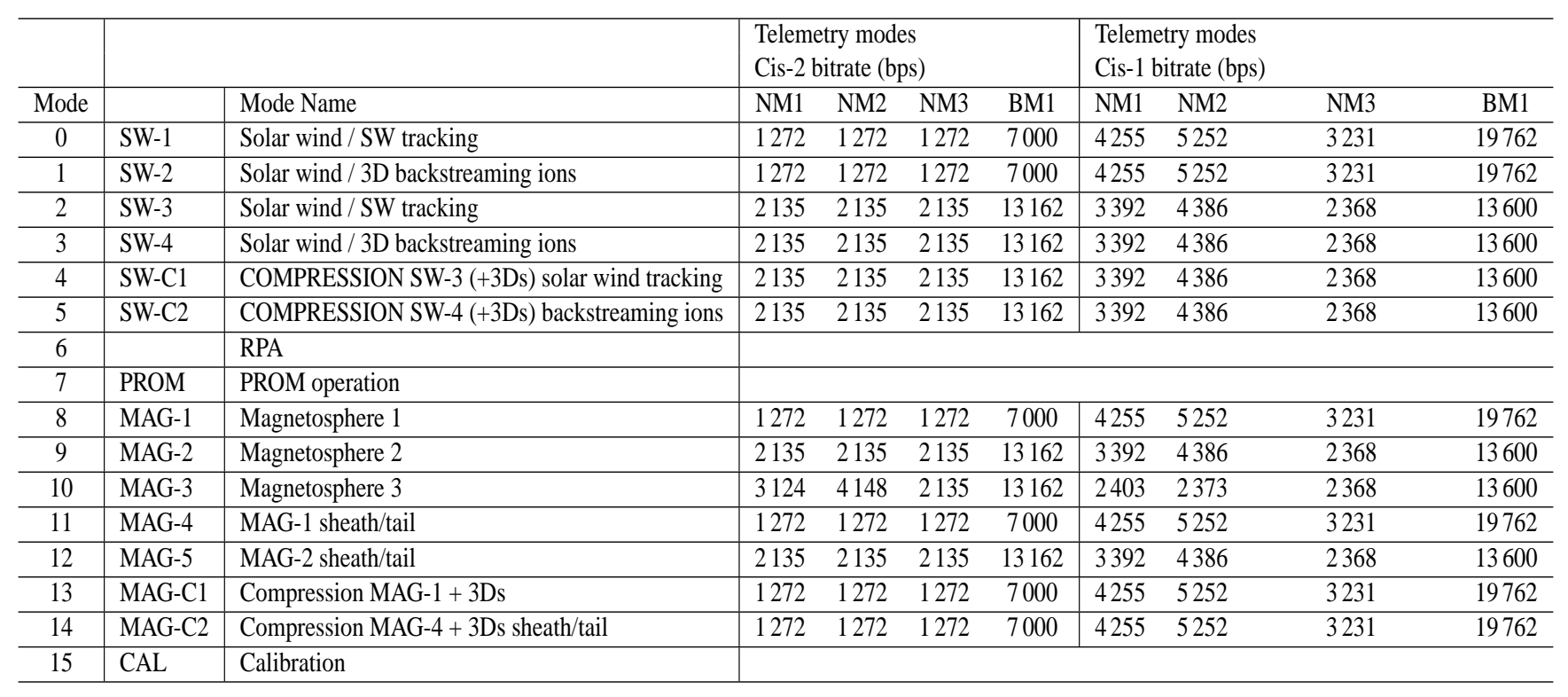

NM1: $5527 \mathrm{bit} / \mathrm{s}$ (Normal mode)

NM2: 6521 bit/s (Ion mode)

NM3: 4503 bit/s (Electron mode)

BM1: $26762 \mathrm{bit} / \mathrm{s}$ (Normal burst mode)

BM2: 6546 bit/s (WEC/WBB TR mode)

BM3: 29456 bits/s (Event memory readout)
Calibration mode at the beginning and the end of each observation period

(Detailed 3D distributions without combination of polar anodes) pendent parameters are copied from the defaults on processor reset (this is called the "Fixed Table"). The "Operational Mode Table" is copied from the default table to set up a new mode after commanding. Sometimes it may also be desirable to follow automatic operational mode changes based only on science data (e.g. moments) collected by the instrument. The "Telemetry Allocation Table" is a subset of the Operational Mode Default Table; when the telemetry rate changes, the appropriate Telemetry Allocation Table is copied from the default table for the new rate and the current operational mode.

The CIS- 1 and CIS-2 instruments have separate tables, but, of course, are controlled by the same telemetry rate and operation mode commands.

\subsection{Ground science data processing}

The CIS raw telemetry is pipeline-processed at the French Cluster Data Centre at CNES, Toulouse, where CESRdeveloped software is running. Level-1 and Level-2 data products are thus systematically generated. Level-1 files correspond to decommutated and decompressed data, organised in flat files, in full time resolution, with one file per spacecraft-day-data product. Level-2 files are the CDF files in physical units, and they include the density for the major ion species, bulk velocity, parallel and perpendicular temperature. These files are organised following the Cluster Science Data System (CSDS) recommendations, and they populate two data bases: the Prime Parameter Data Base (PPDB: four spacecraft, $4 \mathrm{~s}$ resolution) and the Summary Parameter Data Base (SPDB: 1 spacecraft, 1 min resolution). The contents of these data bases are distributed to other National Data Centres on a daily basis. The PPDB are accessible to the whole Cluster community, and the SPDB is a public domain. Due to their broad accessibility and to the quality of their data products, these data bases must permit joint analysis of plasma parameters from several instruments, further enhancing the science return of the Cluster mission. Caveats concerning the limitations of the data are systematically added to the CSDS files, and the users are strongly encouraged to read these caveats prior to any study.

Higher level data processing (Level-3) is performed at the CIS PI and Co-I institutes, using interactive software that reads the Level-1 and Level-2 files, and the calibration files. This software has been developed at CESR ("CL" software) and at IFSI ("IFSIDL" software); it is modular and objectoriented, and has been designed to take into account the data collection pattern specific to each CIS mode.

The health and the performance of the CIS instrument are monitored at various levels by using files retrieved via the network from the Cluster Data Disposition System (DDS), both at JSOC and at CESR. 
Table 8. CIS 2 Scientific Modes

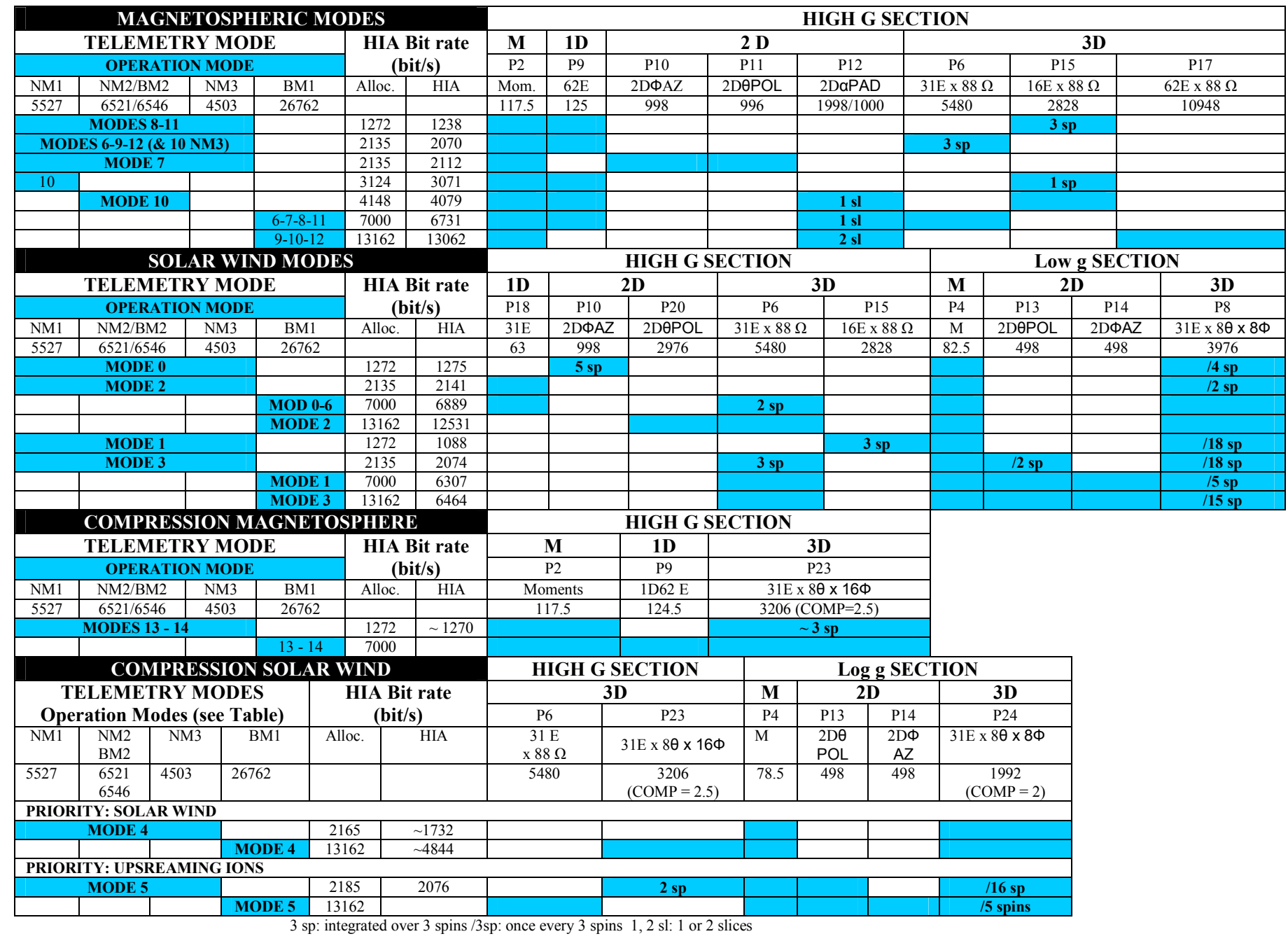

\section{First in-flight CIS results}

The first CIS instrument tests began early in September 2000 in the time frame of the commissioning period. This period for CIS ended around 20 January 2001. The commissioning period was scheduled to test and to adjust all the models, and to test the telemetry products in the different modes, which is extremely complex for CIS. The scientific measurements had no priority during this period and the technical tests could limit the interest of the measurements in some cases.

As the conclusion of this commissioning, 3 CIS instruments were working very well. However, on spacecraft 2, a problem of power consumption appeared very rapidly, showing that something was wrong on the primary side of the Low Voltage Power Converter. After several tests were completed, another test was tried on 25 October 2000, but after $3 \mathrm{~min}$, the spacecraft current limiter turned off the CIS instrument. Other tests will be tried later in the mission, but the problem seems to be very serious and the probability of recovering the spacecraft 2 CIS instrument is small. With 3 remaining spacecraft, the CIS instruments are still capable of giving important information on the composition, fluxes, velocities, dynamics and temperatures of the ions. In connection primarily with FGM, PEACE and RAPID instruments, many new and important results will be obtained with the Cluster mission.

\subsection{Example of validation of the moment calculations}

On this day, CIS was operating on spacecraft 3 and 4 . The general features of the mixed ion region are quite similar for the two spacecraft. Even with a zoom in time (Fig. 28), no significant differences are seen between the two spacecraft. This indicates that the mixed ion region has spatial scales much larger than the spacecraft separation of $650 \mathrm{~km}$.

In Fig. 18, an example of outbound magnetopause crossing by the Samba spacecraft on 19 December 2000 is shown. In this figure, an Energy-Time spectrogram measured by the HIA sensor, the density calculated on board by HIA and the waves measured by the WHISPER instrument (P. Décréau, private communication) are also shown. On the density plot measured by HIA are 3 points showing the electron den- 


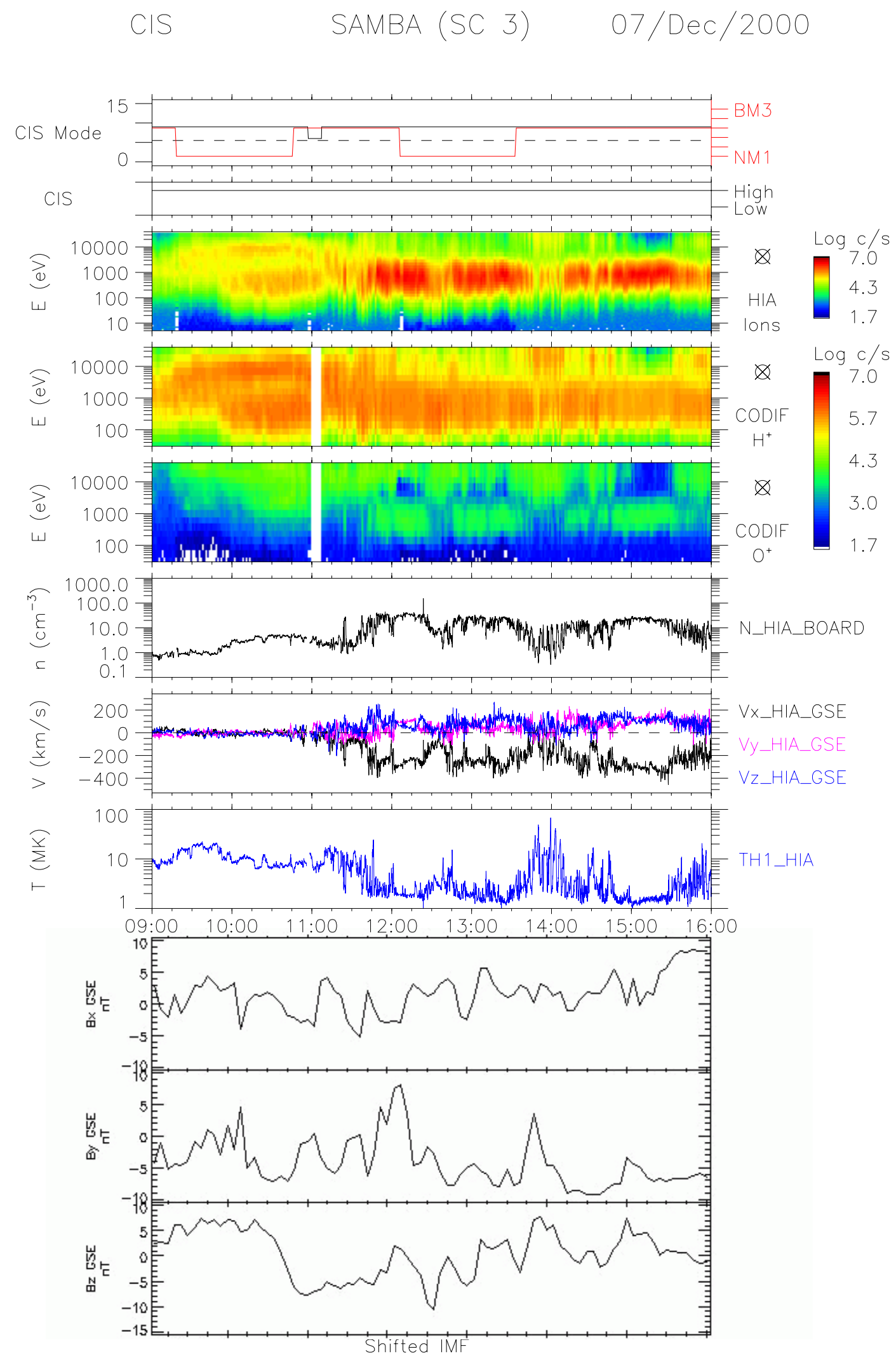

Fig. 27. 7 December 2000 magnetopause crossings between 09:00 and 16:00 UT as seen by spacecraft 3; from top to bottom: Instrument modes, energy-time spectrograms of HIA ions, CODIF $\mathrm{H}^{+}$, and CODIF $\mathrm{O}^{+}$, HIA density, HIA velocity, and shifted IMF (from ACE,

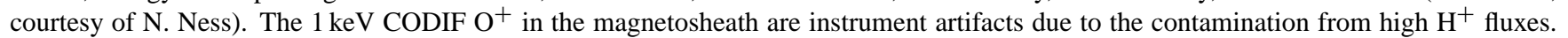
The $10 \mathrm{keV} \mathrm{O}^{+}$are true $\mathrm{O}^{+}$counts. 


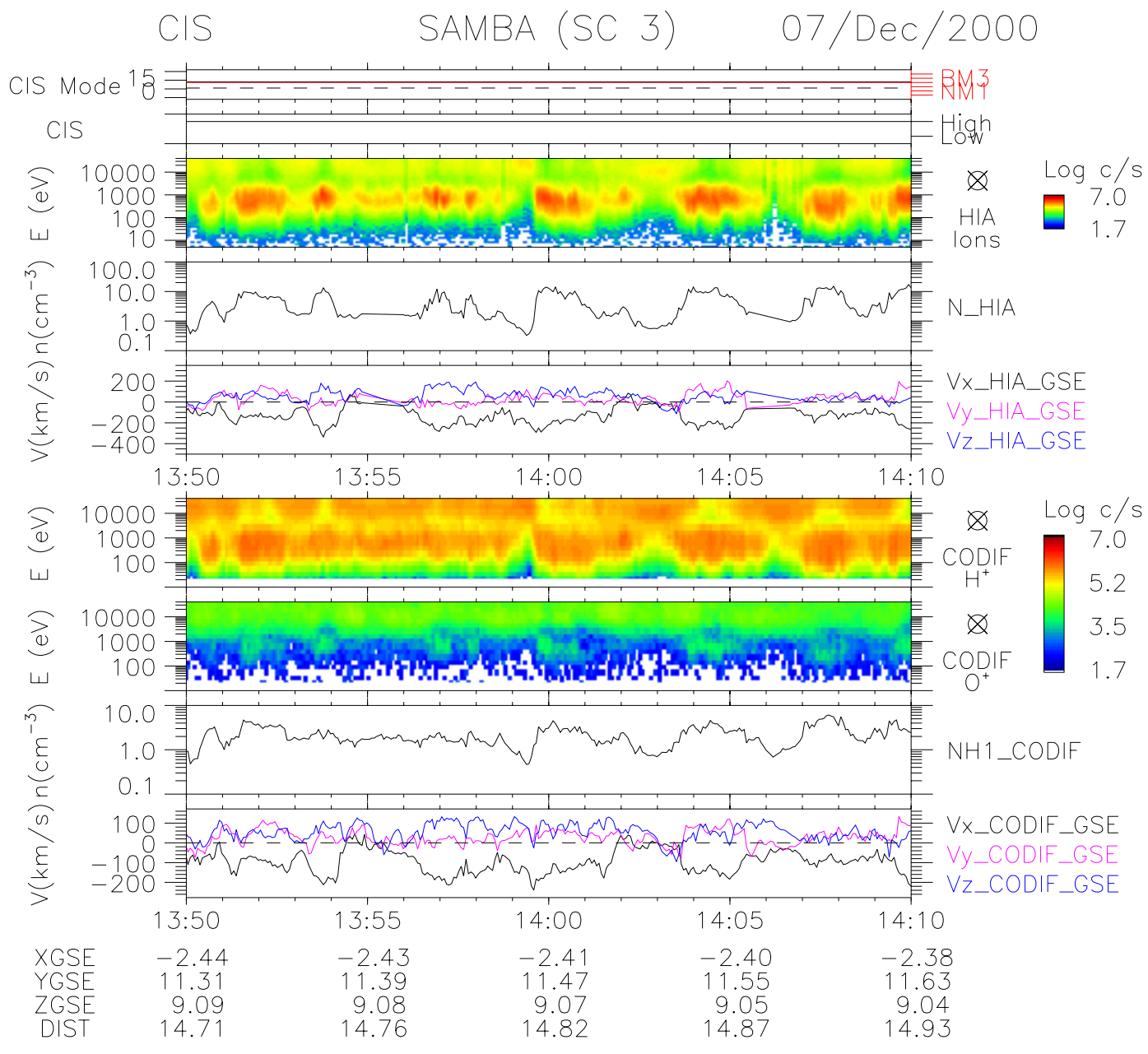
CIS-CODIF
TANGO (SC 4)
07/Dec/2000

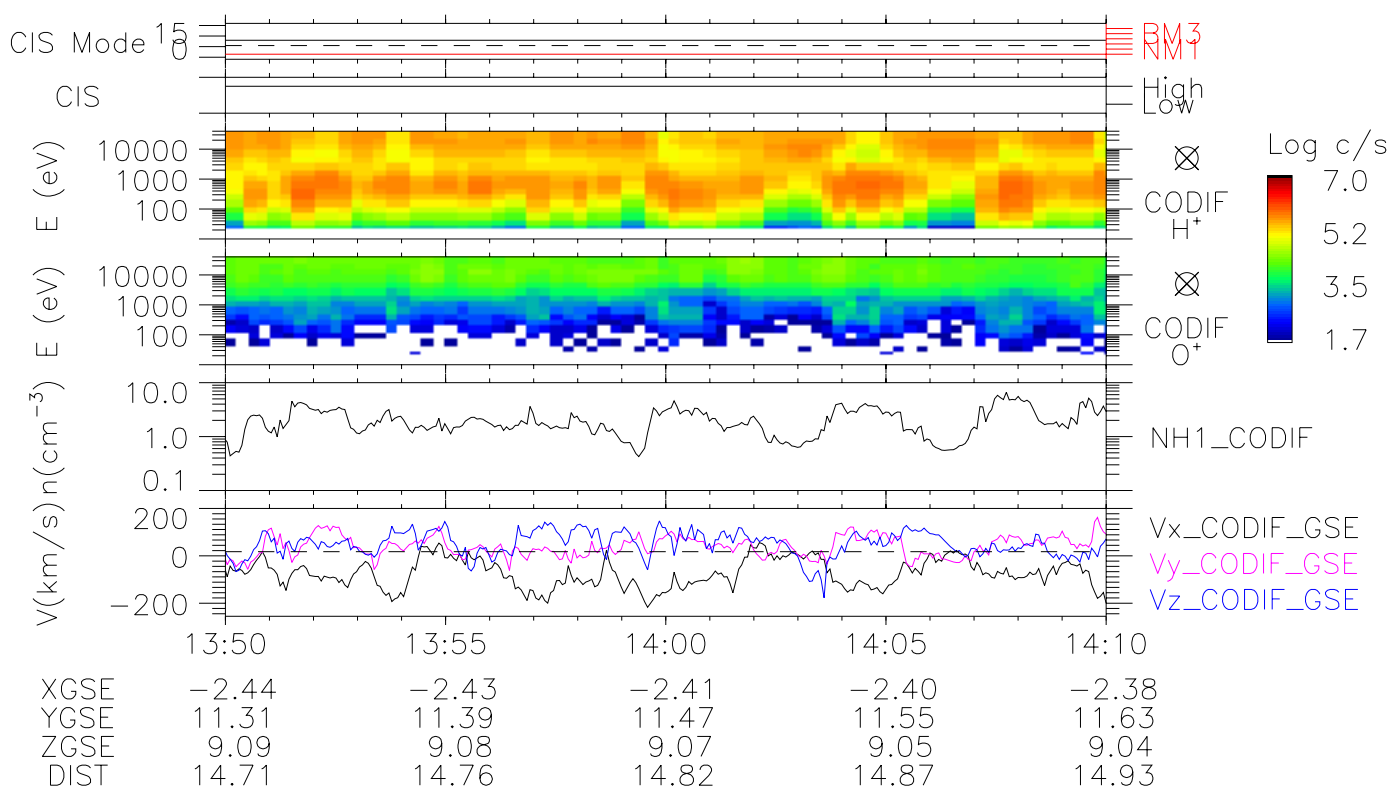

Fig. 28. HIA and CODIF measurements on spacecraft 3 and CODIF measurements on spacecraft 4, on 7 December 2000 between 13:50 and 14:10 UT. See caption of Fig. 22 for the description of the measurements. 


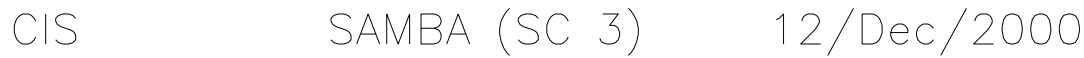
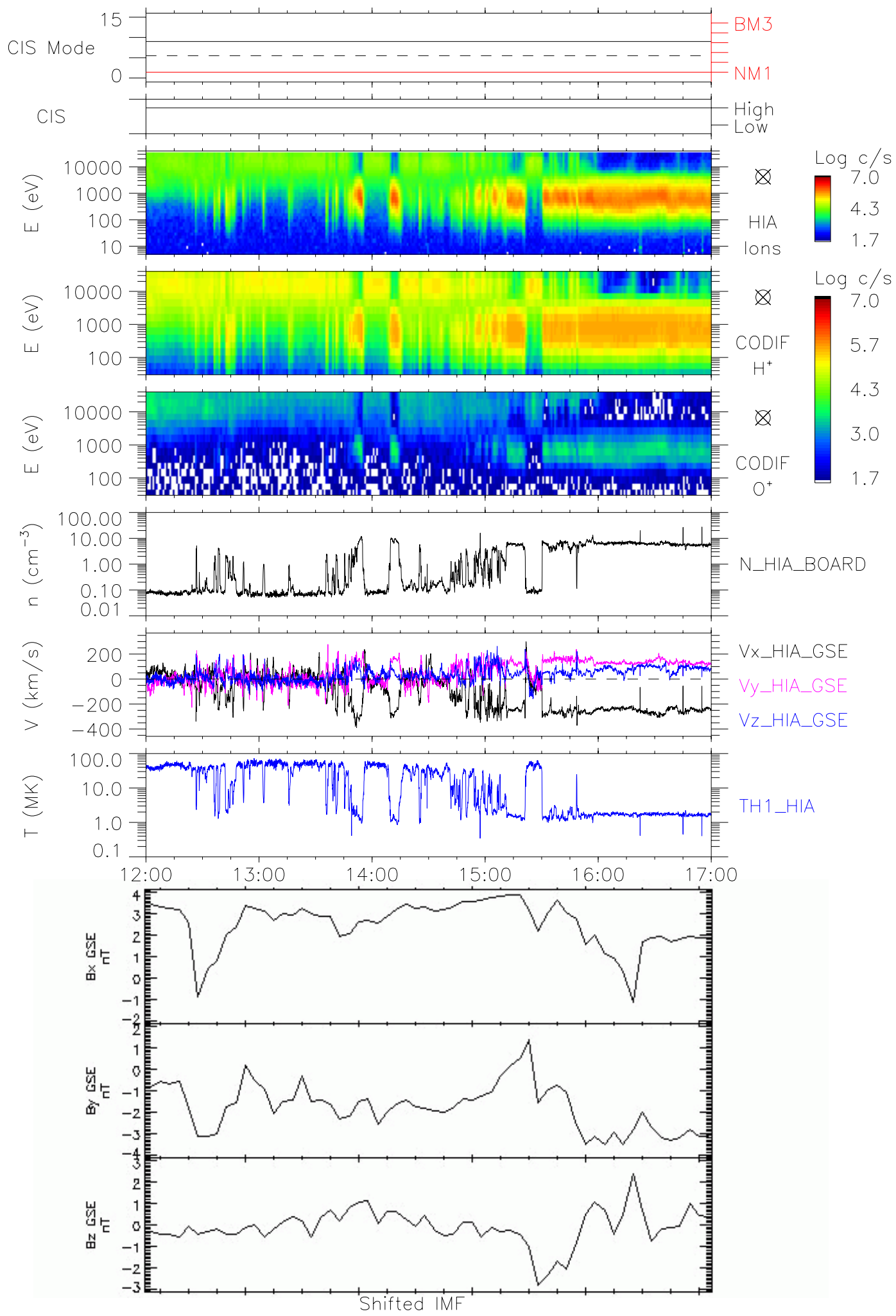

Fig. 29. 12 December 2000 magnetopause crossings between 12:00 and 17:00 UT as seen by spacecraft 3; from top to bottom: instrument modes, energy-time spectrograms of HIA ions, CODIF $\mathrm{H}^{+}$, and CODIF $\mathrm{O}^{+}$, HIA density, HIA velocity, and shifted IMF (from ACE, courtesy of N. Ness). The $1 \mathrm{keV}$ CODIF $\mathrm{O}^{+}$in the magnetosheath are instrument artifacts due to the contamination from high $\mathrm{H}^{+}$fluxes. The $10 \mathrm{keV} \mathrm{O}^{+}$are true $\mathrm{O}^{+}$counts. 

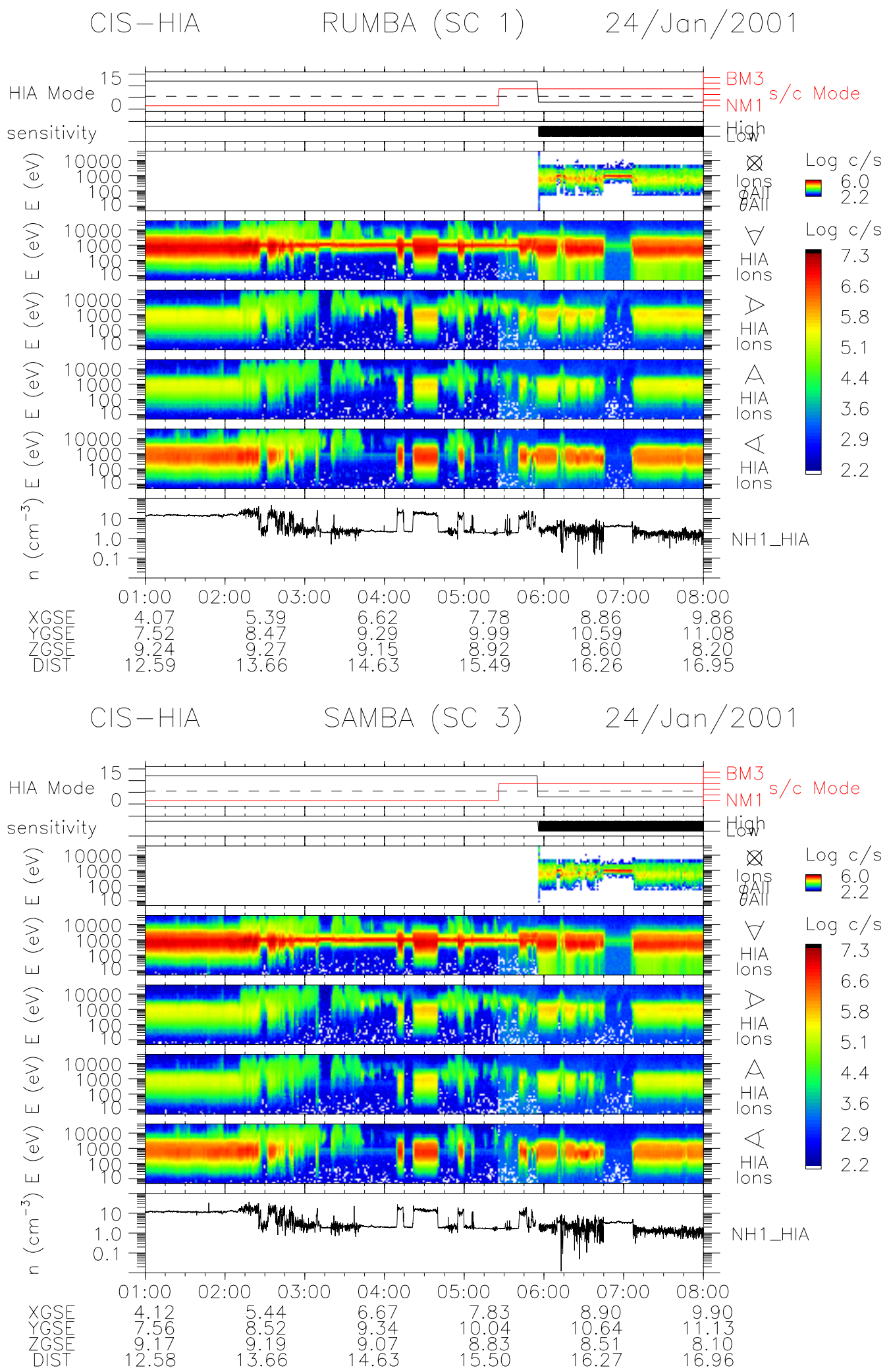

Fig. 30. Measurements of HIA with spacecraft 1 and spacecraft 3 on 24 January 2001, in the magnetosheath and the solar wind, between 01:00 and 08:00 UT. Upper panel shows HIA measurements with spacecraft 1 and lower panel HIA measurements with spacecraft 3. In each panel, from top to bottom: HIA telemetry modes and sensitivities, Low Side (used only in solar wind mode) energy-time spectrogram (integrated over $45^{\circ} \times 45^{\circ}$ centered in the solar wind direction), High Side energy-time spectrograms measured in the sunward, dusk, anti-sunward, dawnward looking directions, and density. 

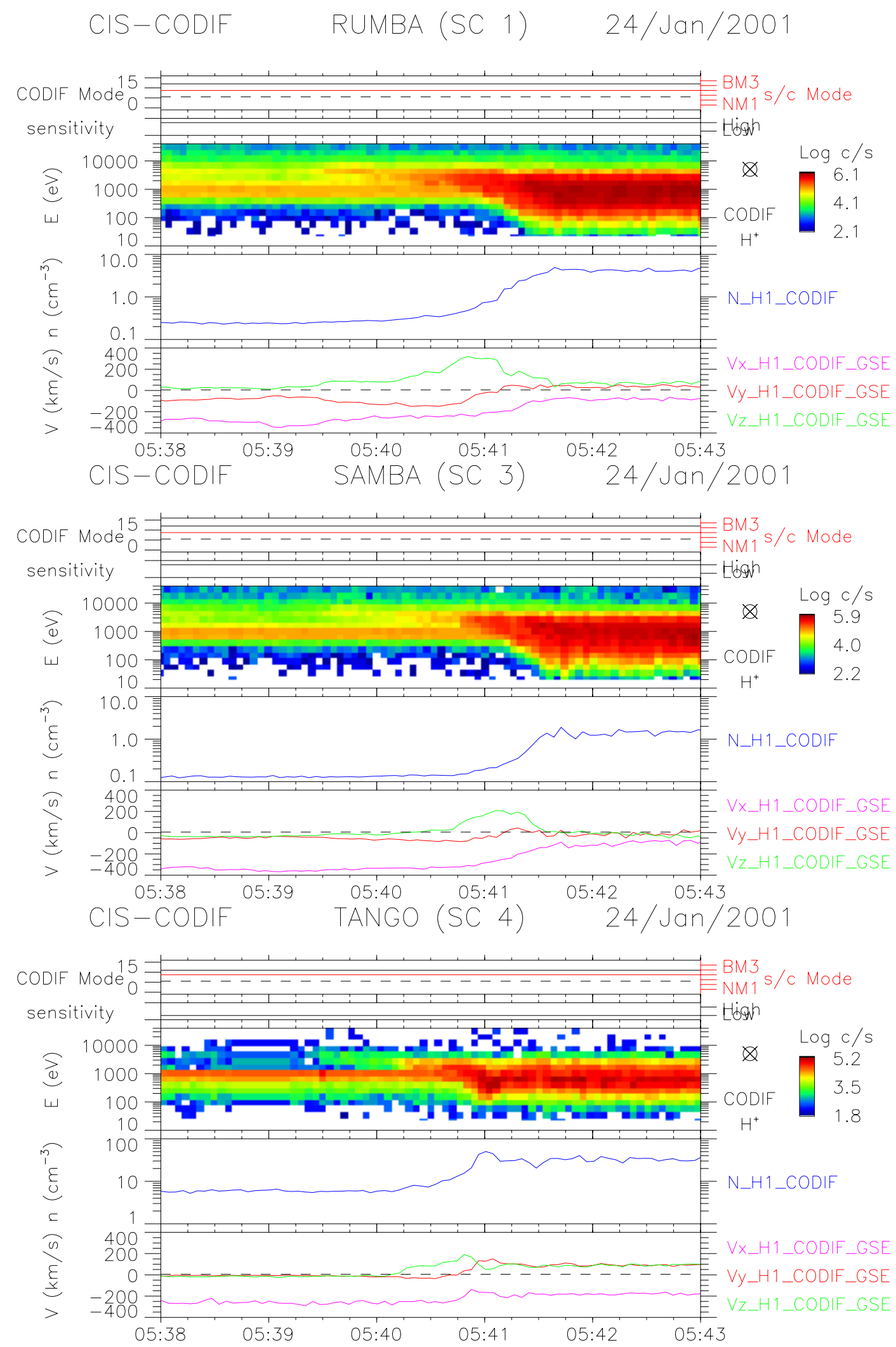

Fig. 31. Entry in the magnetosheath of spacecraft 1, 3, and 4 on 24 January 2001, between 05:38 and 05:43 UT as seen by CODIF. The three panels show CODIF telemetry modes and sensitivities, energy-time spectrogram integrated over 4 , density and GSE velocity components. Note that on spacecraft 4 (lower panel), CODIF is used in low sensitivity mode and the density is correct, while on spacecraft 1 (upper panel) and spacecraft 3 (middle panel), CODIF is used in high sensitivity mode and the measured densities are not correct due to saturation effects. 

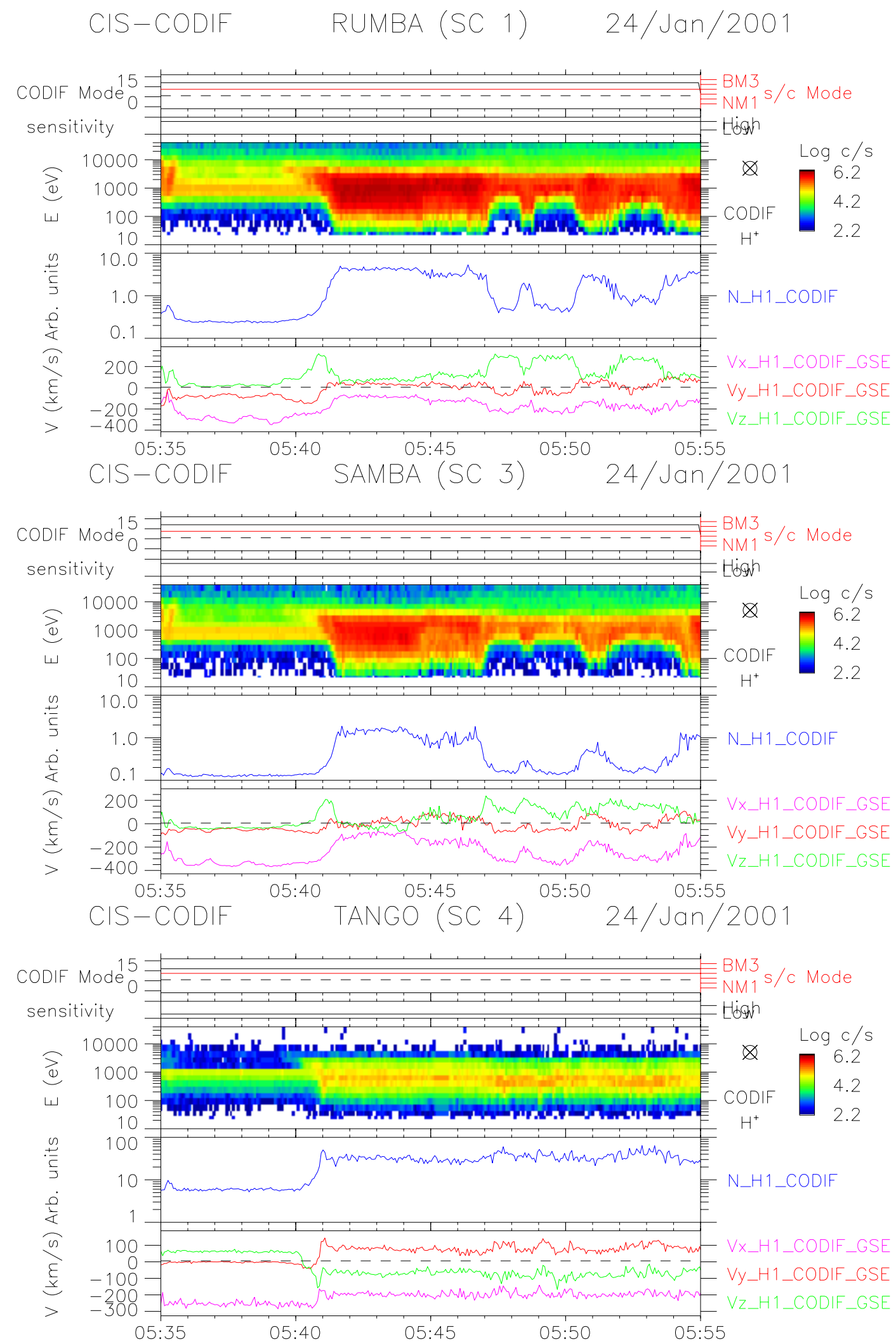

Fig. 32. CODIF measurements on board spacecraft 1, 3, and 4 on 24 January 2001, between 05:35 and 05:55 UT. See Fig. 31 caption for the details of the measurements. 
CIS-HIA RUMBA (SC 1) 19/Feb/2001

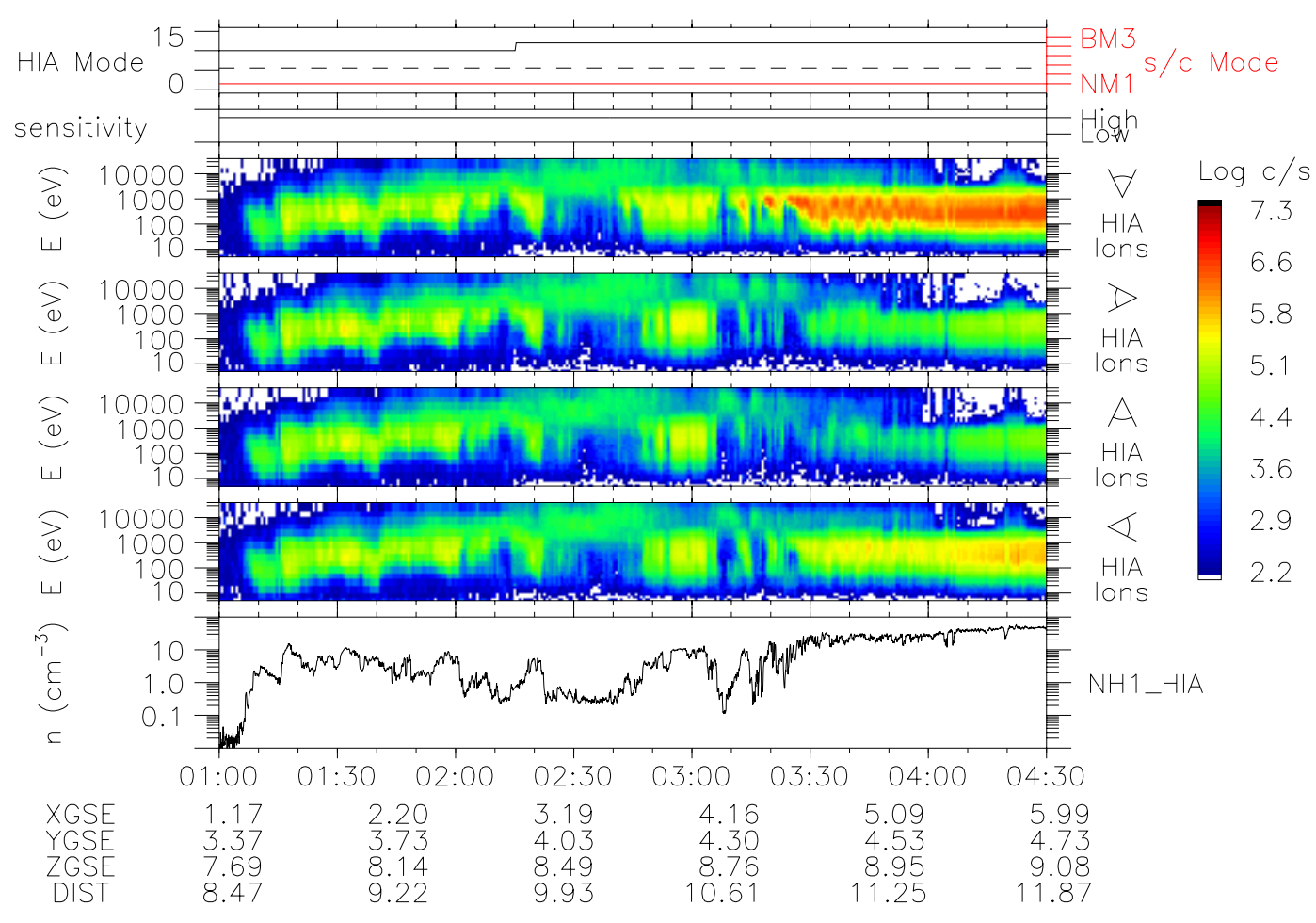

CIS-HIA SAMBA (SC 3) $\quad 19 /$ Feb/2001

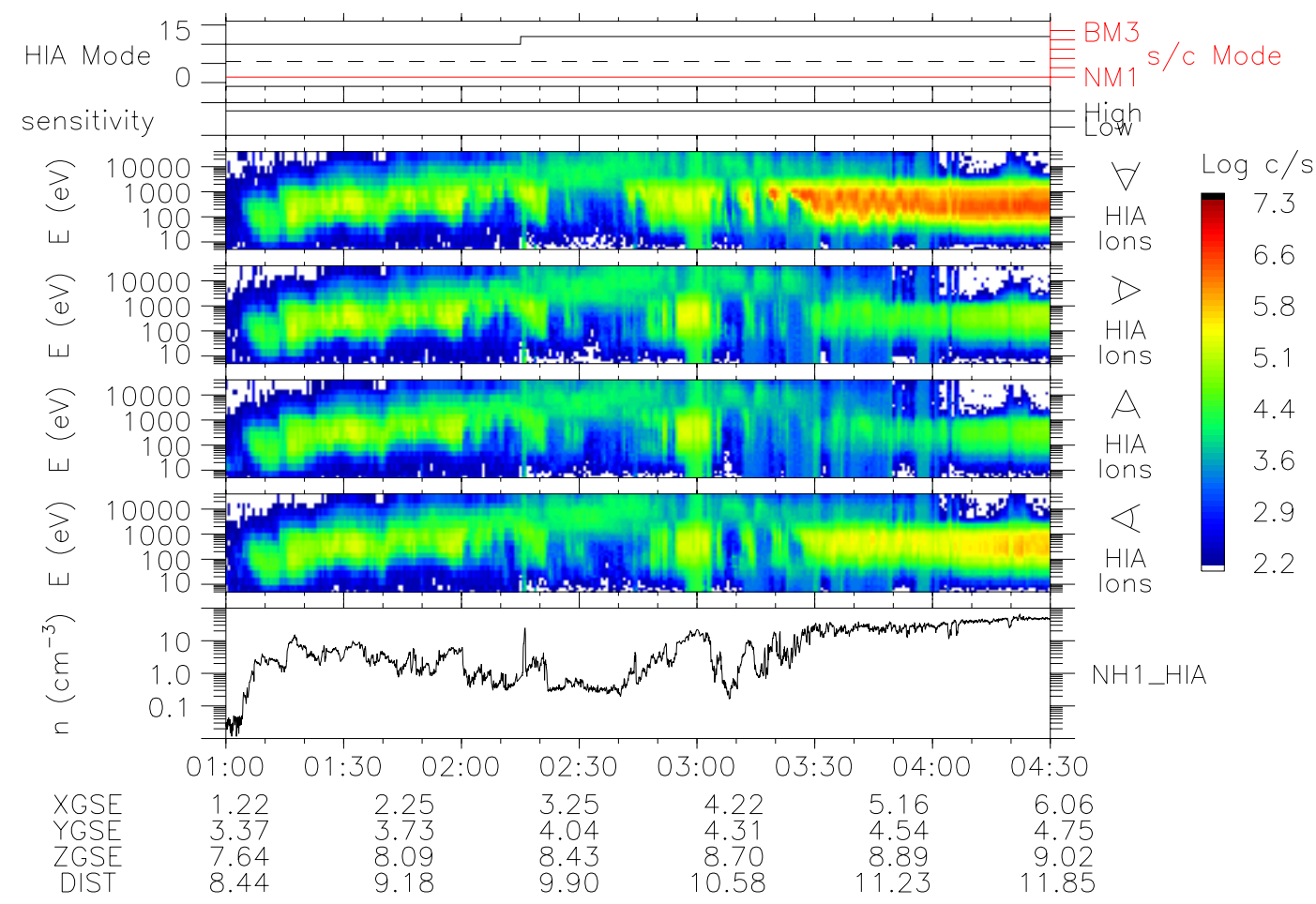

Fig. 33. HIA measurements on board spacecraft 1 and 3, on 19 February 2001, between 01:00 and 04:30 UT. Upper panel shows HIA spacecraft 1 measurements and lower panel HIA spacecraft 3 measurements. The two panels show from top to bottom: telemetry modes and sensitivities, energy-time spectrogram measured in the sunward, dusk, anti-sunward, dawnward looking directions, and density. 


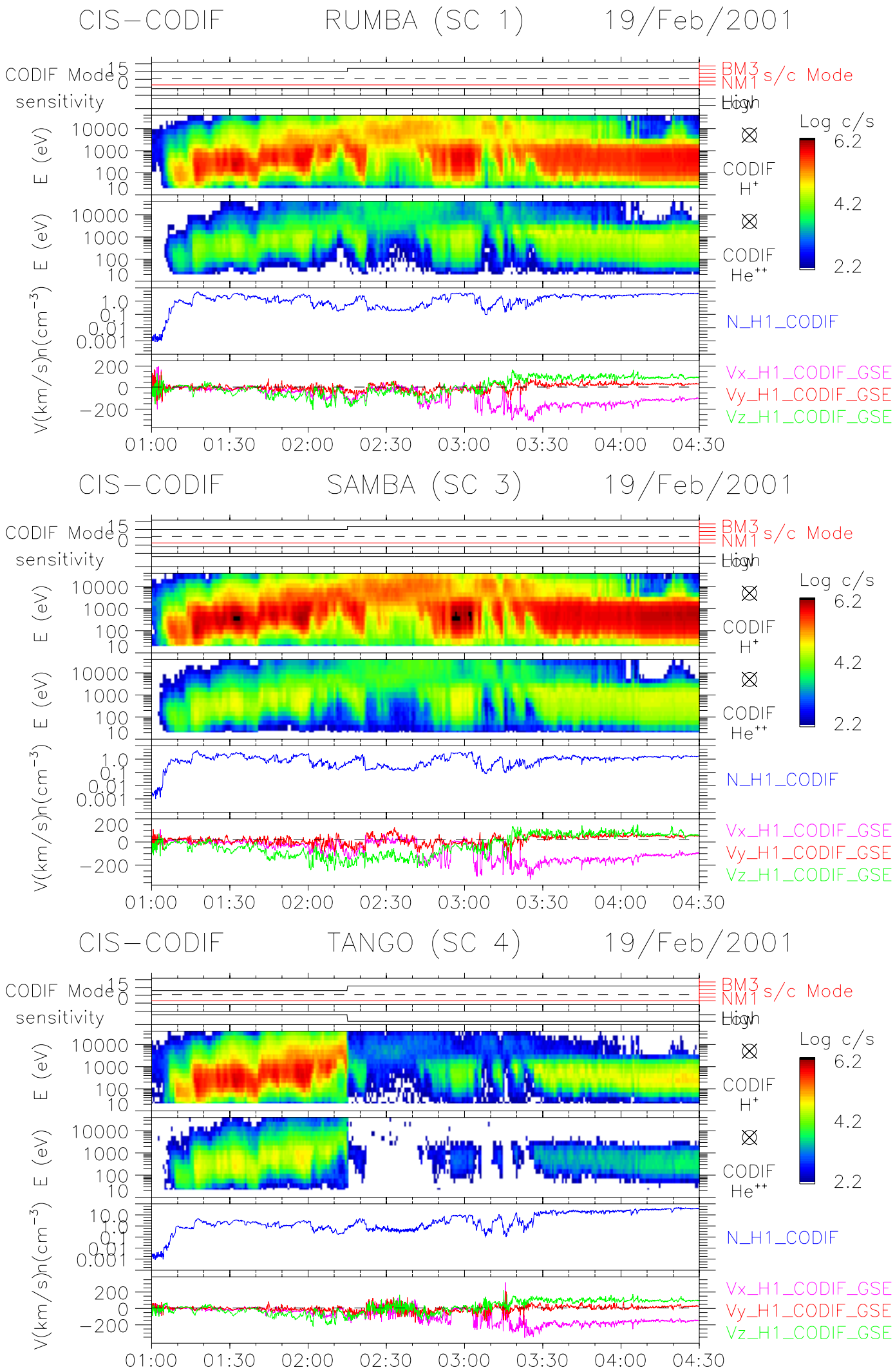

Fig. 34. CODIF measurements on board spacecraft 1 (upper panel), 3 (middle panel) and 4 (lower panel), on 19 February 2001 , between 01:00 and 04:30 UT. The three panels show CODIF telemetry modes and sensitivities, $\mathrm{H}^{+}$and $\mathrm{He}^{++}$energy-time spectrograms integrated over $4 \Pi$, density and GSE velocity components. 

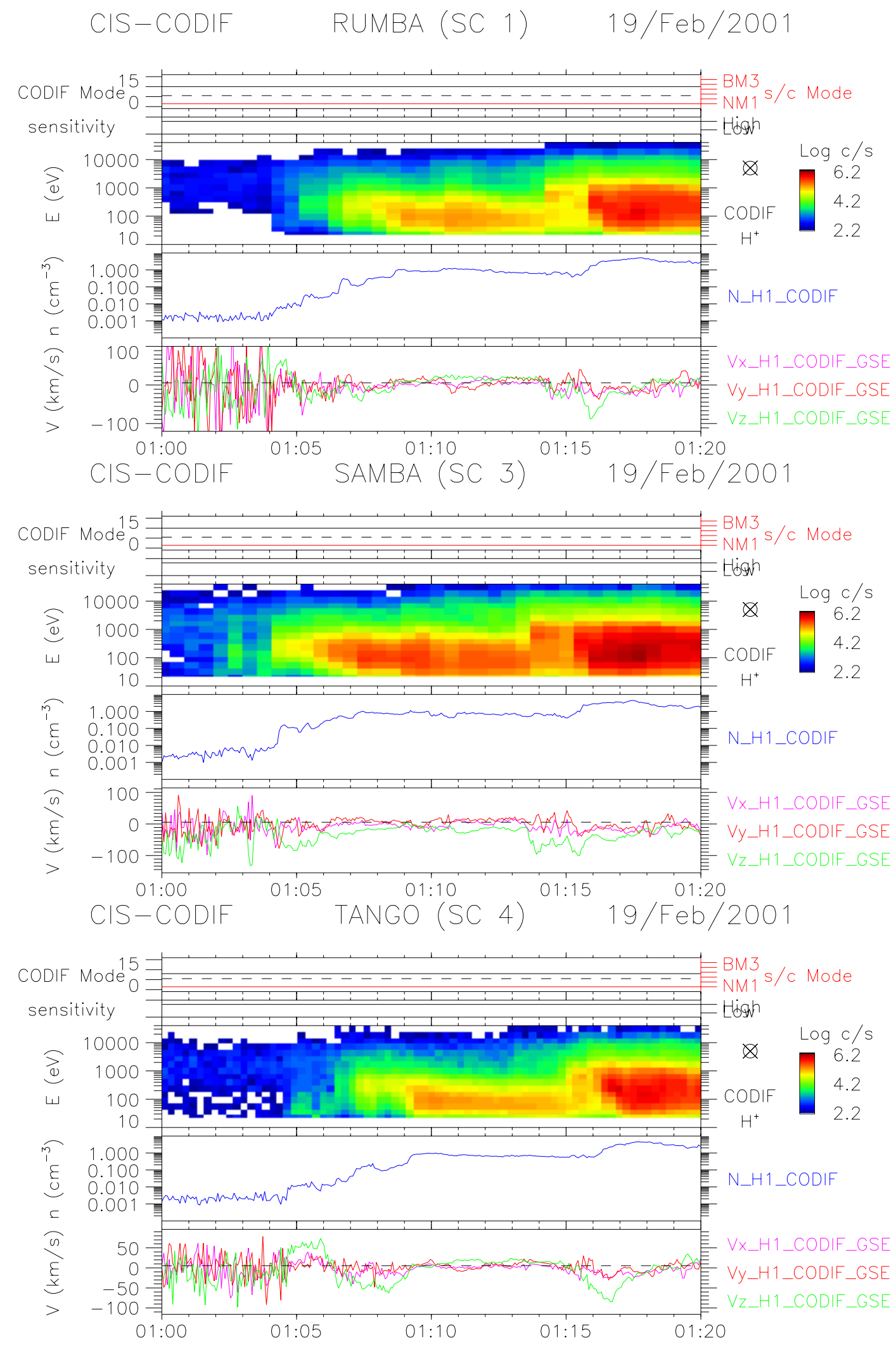

Fig. 35. CODIF measurements on board spacecraft 1, 3 and 4, on 19 February 2001, between 01:00 and 01:20 UT. See Fig. 34 caption for the details of the measurements. 
sity deduced from the wave instrument. Before this magnetopause crossing, the density was too small to be measured by the wave instrument. In the magnetosheath, the agreement is excellent between the two instruments.

\subsection{Importance of the calibrations}

The Cluster PPDB and SPDB data sets (see Sect. 5.4) are created from the moments generated on board the spacecraft. The calculation depends on the efficiencies of the instrument as a function of energy and angle. These efficiencies change with time due to MCP gain fatigue, so there are times when the correct efficiencies are not being used in the onboard calculation. An example of this is shown in Fig. 19, from 31 January 2001. During this time, the efficiencies for CODIF on spacecraft 3 have changed, but no correction had yet been implemented. Fig. 19 shows the velocities from both HIA and CODIF during a time when the spacecraft was in the outer magnetosphere, and the velocities should be low. Below the counting rate of CODIF, for $\mathrm{H}^{+}$ions, as a function of the energy, the next three panels show the onboard velocities. The CODIF instrument shows a large velocity of $-300 \mathrm{~km} / \mathrm{s}$ in the $Z$-direction, while the HIA instrument shows nearly a zero velocity. This is the result of the wrong efficiency coefficients in the CODIF onboard calculation. The other six panels (bottom) show the velocities calculated using the 3D distribution functions, and correct efficiencies. For each direction, CODIF is shown first, and then HIA. Now the two instruments agree very well, and the $Z$-velocity from both instruments is close to zero. Times such as these will be noted in the "caveats" of the PPDB and SPDB data sets. When the onboard moments have problems, a member of the CIS team should be contacted to obtain moments from the 3D distributions.

MCP gain fatigue is a slow, irreversible process, but which can be compensated by raising the MCP high voltage. This operation has been performed during the first semester of 2001, once for CODIF (spacecraft 3) and once for HIA (spacecraft 1 and 3).

\subsection{Example of validation of the data compression}

In Fig. 20, are shown simultaneous CODIF measurements from 01:54 UT to 02:23 UT on 23 February 2001, for spacecraft 1 and 3 are shown. These measurements are identical on the two spacecraft during this period, excepted that CODIF data are not compressed for spacecraft 3 and they are compressed for spacecraft 1 . The data compression works very well. Then, for the same telemetry allocation, if the general results are identical for the two spacecraft, the compressed mode gives a better time resolution and the compressed data give access to more detailed structures.

6.4 Example of central plasma sheet measurements on 30 September 2000

The central plasma sheet was crossed only at the beginning of the commissioning phase. On 30 September 2000, CIS was functioning for spacecraft 3 and 4, and simultaneous measurements of the entry and exit of CPS, on the dusk side, were obtained. Figure 21 shows $4.5 \mathrm{~h}$ of HIA and CODIF measurements with spacecraft 3 from 02:45 UT to 07:15 UT. During this period, IMF $B_{Z}$ was negative and a small substorm was detected around 05:30 UT. In the CPS, fluxes were quite isotropic. Several short CPS excursions were detected before the main entry and around 06:00 UT, the spacecraft left the CPS before coming back with several fluctuations. Energies were typically between $1 \mathrm{keV}$ and $10 \mathrm{keV}$. Very similar results were obtained with spacecraft 4 . Details of short entries in the boundary layer and the CPS are shown for the two spacecraft in Fig. 22. The general and detailed structures are very similar between the two spacecraft. Figure 23 shows the simultaneous measurements for the event around 03:19 UT. It is not possible to show the significant differences between the two spacecraft for this event. However, in another example, a small delay (about $12 \mathrm{~s}$ ) between the two spacecraft can be detected (Fig. 24). For the study of the CPS, the interdistance between the two spacecraft appears to be a little too small. Two examples of 3D distribution functions measured by CODIF on spacecraft 3 are shown in Figs. 25 and 26. At 03:03:31 UT, counterstreaming ions are seen in the sunward and in the anti-sunward directions (positive and negative $V_{X}$ ) while at 06:17:23 UT, strong sunward directed ions are detected in addition to a small, slow beam in the $V_{Y}, V_{Z}$ plane.

6.5 The occurrence of a mixed magnetosheath-plasma sheet ion region immediately earthward of the lowlatitude boundary layer (LLBL)

In this section, we study two passes by spacecraft 3 across the dusk flank mid-latitude magnetopause (MP). On one pass (7 December 2000), an extended stagnant mixed ion region was detected. On another pass (12 December 2000) along essentially the same trajectory, the region immediately earthward of the MP/LLBL was the more typical single population hot plasma sheet. The observations were practically identical for spacecraft 4 on these days.

Figure 27 shows an outbound pass on 7 December 2000 by spacecraft 3 from the plasma sheet to the magnetosheath, crossing the mid-latitude dusk flank magnetopause. Panels (c-e) show that the plasma sheet ion distribution before 09:30 UT consists of a single high energy population in both $\mathrm{H}^{+}$(panel d) and $\mathrm{O}^{+}$(panel e), while the magnetosheath proper (for instance, at 15:00 UT) contains a single colder population. Multiple crossings of the MP/LLBL occurred at 11:15-11:40 UT and at 13:45-14:08 UT and these boundary regions are recognized by the presence of tailward flowing mixed magnetosheath-plasma sheet ions (panels $\mathrm{c}$ and g). The region of interest here is the $1 \mathrm{~h}$ interval 10:11 UT, where spacecraft 3 encountered an extended period (over $2 \mathrm{~h}$ and $2 R_{E}$ in GSE-Y) of mixed low and high energy ions. This region is distinguishable from the MP/LLBL by its stagnant plasma. $\mathrm{O}^{+}$is present in the high energy population, but absent in the low energy component, indicating that high en- 
ergy $\mathrm{H}^{+}$and $\mathrm{O}^{+}$are of magnetospheric origin, while the low energy component comes from the magnetosheath. The ion density (panel $\mathrm{f}$ ) in the mixed ion region $\left(\sim 3 \mathrm{~cm}^{-3}\right)$ is considerably higher than that of the plasma sheet $\left(\sim 1 \mathrm{~cm}^{-3}\right)$, while its temperature (panel h) is lower. Finally, the mixed ions were detected when the IMF (panel i-k) was strongly northward (IMF $B_{Z} \sim 5 \mathrm{nT}$ ) and the solar wind density was unusually high $\left(\sim 17 \mathrm{~cm}^{-3}\right)$.

Figure 29 shows another pass by spacecraft 3 on 12 December 2000 along a similar trajectory. The magnetosheath (for instance, after 15:30 UT) and plasma sheet (before 12:30 UT) properties are typical of these regions and are similar to the 7 December 2000 event. However, adjacent to the dusk mid-latitude magnetopause which was crossed multiple times between 12:30 and 15:30 UT, the only mixed ions observed next to the MP were confined to the thin layers of fast flowing LLBL. Immediately earthward of the fast flowing LLBL, the ions encountered by the spacecraft are the typical single-population hot plasma sheet ions, i.e. no stagnant mixed ions were detected. This pass occurred when the IMF $B_{Z}$ was $\sim 0 \mathrm{nT}$ and the IMF $B_{Y}$ was slightly negative. The solar wind was at its typical $2.5-3 \mathrm{~cm}^{-3}$ level.

A region of stagnant, mixed magnetosheath-plasma sheet ions earthward of the MP/LLBL has been detected by Geotail (Fujimoto et al., 1996, 1998), ISEE-2 (Fuselier et al., 1999), and WIND (Phan et al., 2000). A stagnant cold and dense plasma region earthward of the fast flowing LLBL may also be related to the region termed the "halo" by Sckopke et al. (1981) and later reported by Williams et al. (1985), although these studies did not reveal whether the ions in the stagnant region are LLBL-like (mixed ions) or plasma sheetlike (single population). None of the previous studies could establish the mixed ion entry sites or the entry mechanisms. The tendency for these cold dense ions to occur for northward IMF only arose from statistical surveys (Terasawa et al., 1997).

The two passes presented here were along similar trajectories (with a difference of $2 R_{E}$ in GSM-Z) and in both cases the spacecraft moved from the hot plasma sheet to the magnetosheath, but one pass detected an extended mixed ion region while the other did not. The mixed ion case was detected when the IMF was strongly northward and the solar wind was unusually dense, while IMF $B_{Z} \sim 0 \mathrm{nT}$ and the density was more typical for the case when the mixed ions were not detected. To reveal whether the IMF, solar wind density or other factors determine the presence or absence of a mixed ion region earthward of the MP/LLBL requires a comprehensive survey of Cluster crossings of the flank magnetopause regions. The detection of consecutive regions of the magnetosheath, MP/LLBL and the stagnant mixed ions on 7 December 2000 suggests that the entry site must be at the dusk magnetopause. Finally, the spatial extent of the mixed ion region, which is presently not known, can be determined by Cluster multi-point measurements when the spacecraft separation is sufficiently large $(2000 \mathrm{~km})$.
6.6 Example of measurements in the magnetosheath and the solar wind (24 January 2001)

On 24 January 2001, there was a very interesting case of 3 spacecraft simultaneous measurements, with the CIS instrument on spacecraft 1 having been commissioned some days before. Figure 30 shows HIA measurements on spacecraft 1 and 3 between 01:00 UT and 08:00 UT. The two HIAs are in the magnetosphere mode until about 05:55 UT; then they shifted to solar wind mode. In magnetospheric mode, the solar wind is clearly identified by unidirectional and monoenergetic ions, while in the magnetosheath, the distribution is larger in energy and in angle. In solar wind mode, the solar wind is detected on the small $g$ side, but not on the large $G$ side (the high voltage is truncated in this mode to avoid MCP fatigue), while the magnetosheath ions are detected by the large $G$ side. Multiple bow shock traversals are detected. Figures 31 and 32 present a very interesting 3 spacecraft effect. Between 05:40 and 05:55 UT, the results of CODIF $\left(\mathrm{H}^{+}\right.$counting rate, densities and velocities) show ion gyroradius effects seen at the entry for the 3 spacecraft around 05:40 UT and at the exit for spacecraft 1 and 3 near 05:47 UT. The entry near 05:40 UT (Fig. 31) is detected first by spacecraft 4 , then by spacecraft 1 and slightly after by spacecraft 3. Near 05:47 UT, spacecraft 1 and 3 emerge from the bow shock, while spacecraft 4 remains downstream of the shock all the time. These results are consistent with the geometry of the 3 spacecraft at this time. Therefore, spacecraft 4 is a little nearer to the Earth; it is the first to go into the magnetosheath and to remain inside during this event, while the two other spacecraft enter the magnetosheath later and exit near the shock, as seen between 05:47 and 05:55 UT before returning to the magnetosheath. Thus, the morphology is very stable and the spacecraft configuration enters and exits in formation, i.e. into and out of a fixed stable boundary. This example shows the large interest of multispacecraft measurements.

\subsection{Example of cusp measurements (19 February 2001)}

On 19 February 2001, the Cluster spacecraft have flown into the cusp before going to the magnetosheath. Figure 33 shows HIA data on spacecraft 1 and 3 between 01:00 and 04:30 UT. The general features are quite identical between the two spacecraft. For the same time interval, the $\mathrm{H}^{+}$and $\mathrm{He}^{++}$CODIF data for spacecraft 1, 3, and 4 are shown in Fig. 34. Note that there is a change of sensitivity for CODIF at 02:15 UT on spacecraft 4 (from High Side to Low Side measurements). A zoom of the $\mathrm{H}^{+}$data is shown in Fig. 35 between 01:00 and 01:20 UT.

At 01:05 UT, after having passed the near tail lobes, Cluster spacecraft entered the mid-altitude cusp, where a plasma of magnetosheath origin is clearly seen. Energy distributions and ion composition $\left(\mathrm{H}^{+}\right.$and $\left.\mathrm{He}^{++}\right)$of CODIF data strongly differ between the cusp and the dayside plasma sheet (Fig. 34), with the cusp characterized by the significant presence of the $\mathrm{He}^{++}$ions. This latter region was encoun- 


\section{CIS-CODIF SAMBA (SC 3) 19/Feb/2001 01:18:30.752}
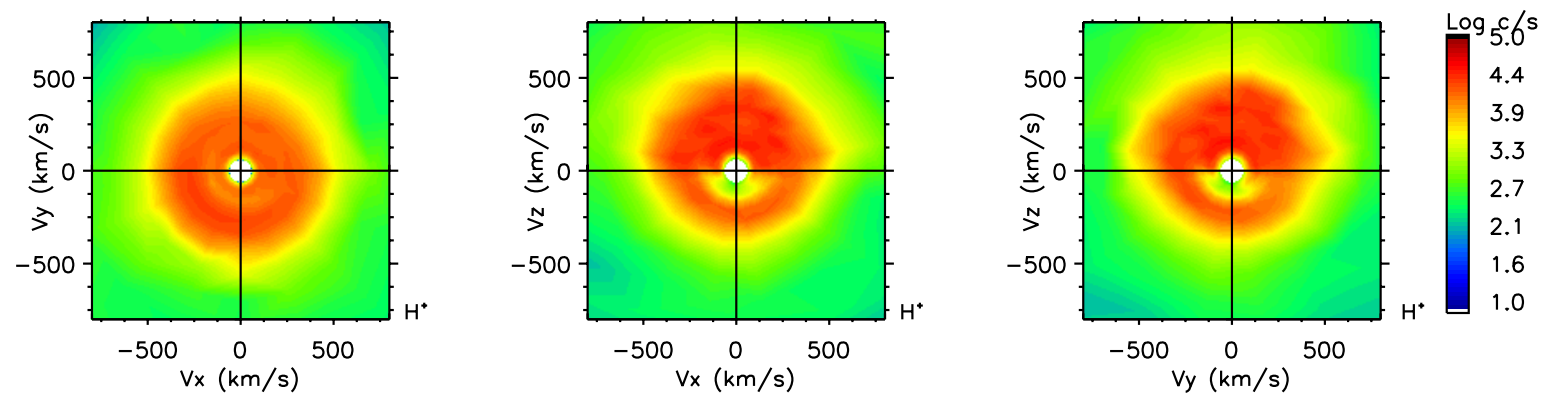

\section{3 spin accumulation}

Fig. 36. $\mathrm{H}^{+}$ions $3 \mathrm{D}$ CODIF measurement on spacecraft 3 on 19 February 2001, at 01:18:30 UT.

tered between 02:20 and 02:50 UT, and occasionally between 03:00 and 03:20 UT before the entry of the spacecraft into the magnetosheath. There is a small delay between spacecraft 3, which first enter the cusp, and spacecraft 1, followed by spacecraft 4 , in agreement with the geometry of the spacecraft tetrahedron (from OVT, not shown); spacecraft 1 and 4 are, however, relatively closer and the time difference between these two spacecraft is small.

During the main mid-altitude cusp traversal (01:05 to 02:20 UT), many injection and time/energy dispersed structures can be seen, similar to the "Cusp Ion Steps" studied by Lockwood and Smith (1992). In Fig. 36, a GSE distribution function from CODIF $\mathrm{H}^{+}$measurements is shown. This figure shows both incoming/downflowing and reflected/upflowing ion populations, as expected in the midlatitude cusp. Its apparent D-shaped structure (Cowley, 1982) may not be a sign of reconnection at the dayside magnetopause. Indeed, it is reversed in the $V_{Z}$ component (assuming that the $\boldsymbol{B}$ field lies roughly in the $-\boldsymbol{Z}$ direction) compared to what would be expected for such a reconnection signature (see also Smith and Lockwood, 1996).

Since the IMF is clearly oriented southward during the whole interval (not shown), the apparent poleward convection feature (since there is a global increase in particle mean energy during the crossing), added to the previous remarks, leads to a possible occurence of a subsolar reconnection, as first proposed by Dungey (1961). Other examples of reconnection signatures as seen by the CIS are presented in more details by Bosqued et al. (2001, this issue).

\subsection{CIS observations in the auroral acceleration region}

The four Cluster satellites are very useful for studying auroral plasma acceleration processes and plasma sheet dynamics at radial distances of 4-6 Earth radii over the nightside south and north auroral zones. Starting from late December 2000, the Cluster orbit pericenter moved into the nightside/tail region, thus allowing for data collection within the auroral oval close to the local magnetic midnight. The four Cluster spacecraft traverse the auroral oval field lines consec- utively at almost the same magnetic local time, separated in time by about $200 \mathrm{~s}$ (spacecraft 1 -spacecraft 3 ).

The CIS team have so far identified over 10 cases of nightside auroral oval traversals by the spacecraft 1,3 and 4 that contain interesting data on the ionospheric upward acceleration of ions. Ion outflow from the auroral regions is a significant plasma source in the magnetosphere (Chapell, 1988; Kondo et al., 1990; André and Yaw, 1997). The four Cluster spacecraft have the unique capability of traversing auroral field lines at almost constant heights above what is statistically conceived as the altitude of auroral plasma acceleration. The consecutive traversal makes it possible, for the first time, to study in situ the temporal/spatial evolution of auroral plasma acceleration processes. Some preliminary findings from the CIS data are as follows:

- The upward acceleration of ionospheric ions is quite dynamic, with small- and medium-scale features varying considerably within $200 \mathrm{~s}$.

- However, the large-scale morphology of the upward acceleration region, and the large-scale "ion inverted V" remains rather constant in the few minutes time scale.

- The field-aligned upward acceleration process is clearly mass dependent, with heavier ions acquiring higher peak energies.

- Ion acceleration clearly continues in the 4-6 $R_{E}$ altitude range, primarily by what appears to be transverse heating/acceleration. Instead of focusing with height (due to the magnetic mirror force), the ion beams continues to be broad.

- Downward plasma sheet ion beams are generally seen in the same region as upgoing ion beams. The downgoing beams have higher energies than the upgoing beams, suggesting that they originate from an acceleration region in the tail (Sauvaud et al, 1999; Sergeev et al., 2000) or, if they originate in the conjugate/opposite hemisphere, this indicates that the ion acceleration process progresses even beyond the altitude of $6 R_{E}$. 


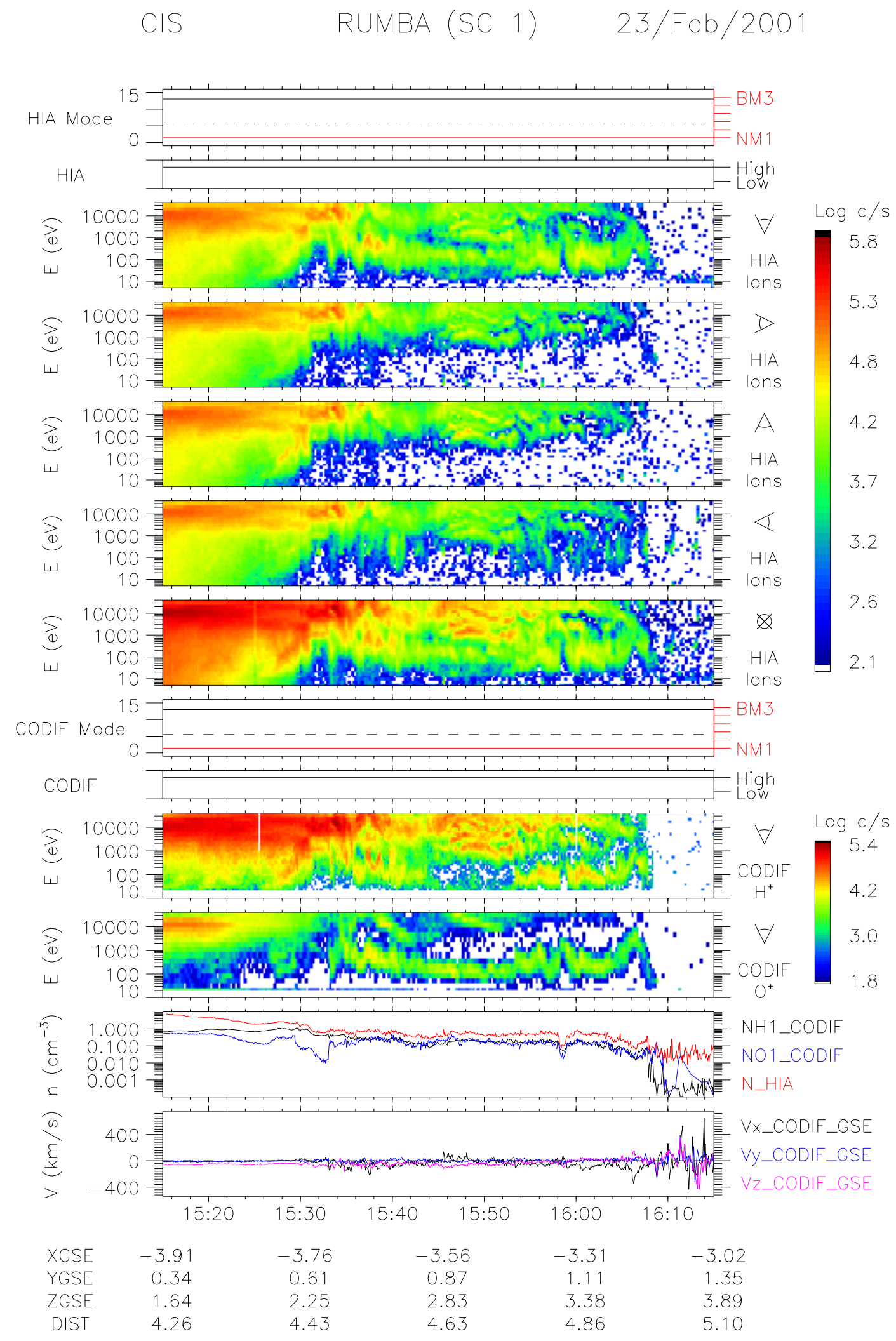

Fig. 37. Traversal of the auroral region by spacecraft 1 on 23 February 2001, as seen by HIA and CODIF between 15:15 and 16:15 UT. HIA measurements (upper panel) give telemetry modes and sensitivities, energy-time spectrograms measured in the sunward, dusk, anti-sunward, dawnward looking directions and energy-time spectrogram integrated over 4 П; CODIF measurements (lower panel) give telemetry modes and sensitivities, energy-time spectrogram for upflowing $\mathrm{H}^{+}$and $\mathrm{O}^{+}$ions, $\mathrm{HIA}, \mathrm{CODIF} \mathrm{H}^{+}$and $\mathrm{O}^{+}$densities and GSE velocity components. 


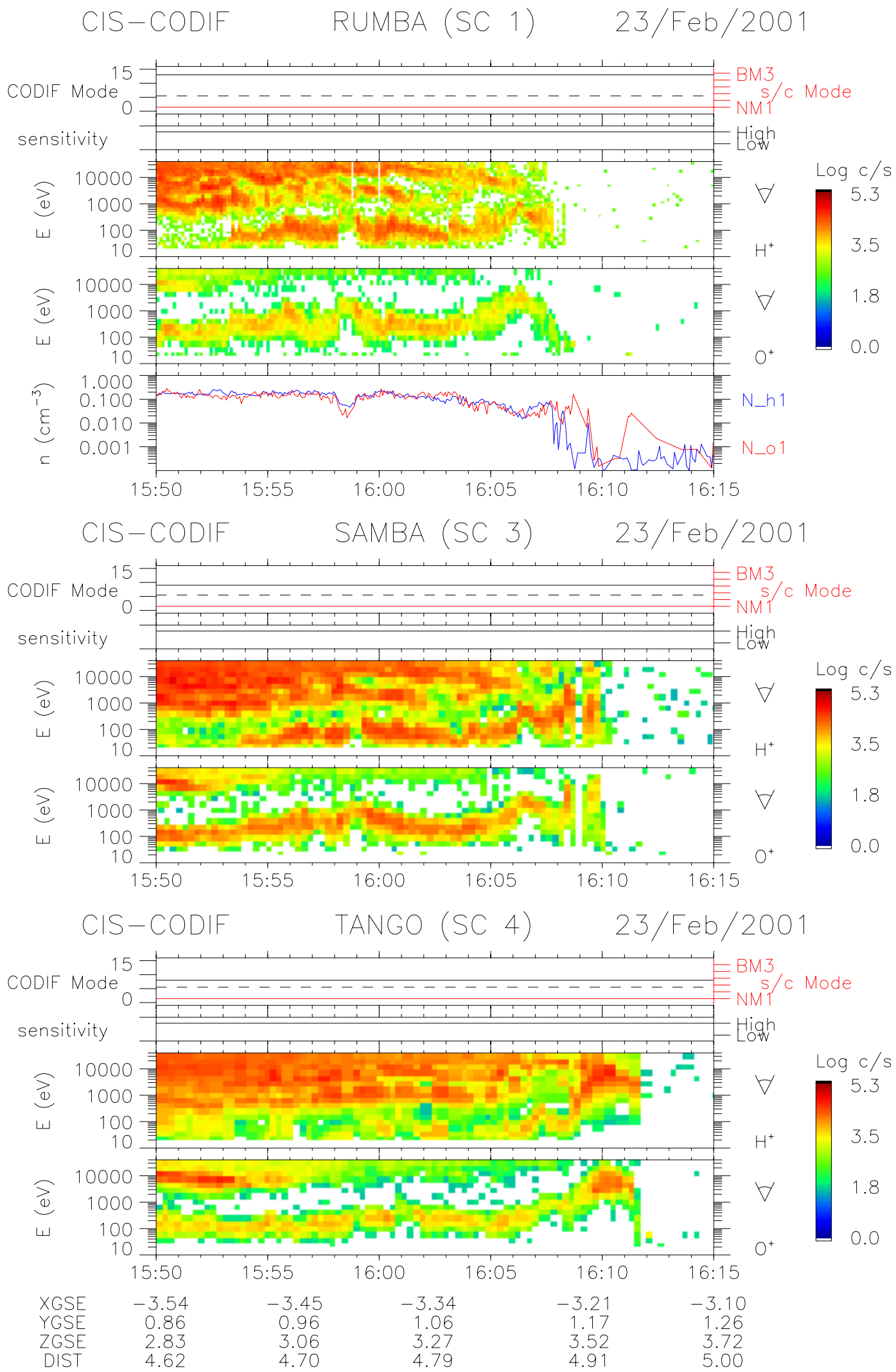

Fig. 38. CODIF $\mathrm{H}^{+}$and $\mathrm{O}^{+}$measurements on spacecraft 1 (upper panel), 3 (middle panel), and 4 (lower panel), on 23 February 2001, between 15:50 and 16:15 UT. Each panel shows telemetry modes and sensitivities, energy-time spectrograms for upflowing $\mathrm{H}^{+}$and $\mathrm{O}^{+}$ions and $\mathrm{H}^{+}$and $\mathrm{O}^{+}$ion densities. 


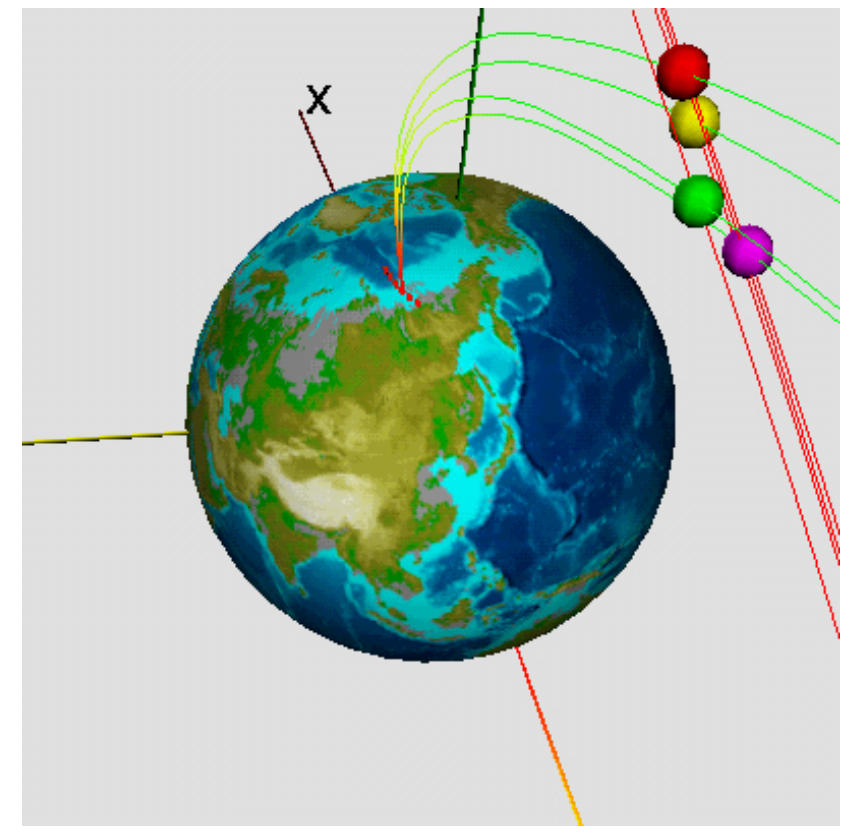

Fig. 39. Positions of the four Cluster spacecraft and geometry of the magnetic field lines deduced from OVT. The four spacecraft are going up; spacecraft 1 is red, spacecraft 2 is green, spacecraft 3 is yellow and spacecraft 4 is violet.

An example illustrating the Cluster spacecraft 1 (Rumba) traversal of the auroral acceleration region is shown in Fig. 37. The two colour spectrogram in the lower most panels represent data taken in the solar direction, which, in the case of Cluster at auroral latitudes near midnight, looks close to the magnetic field lines. At the top are ion fluxes measured by HIA in four angular sectors and integrated over the four sectors. The satellite in this case exited the ring current (left), into the auroral oval, and entered the polar cap region (right). Notice the low energy ion structures for both upflowing $\mathrm{H}^{+}$and $\mathrm{O}^{+}$. In Fig. 38, the evolution of upward ion acceleration can be deduced from the 3 spacecraft measurements, which are in full agreement with the field line geometry given by OVT (Stasiewicz, 2001). Figure 39 shows the position, given by OVT, of the four spacecraft, going in the upward direction, and their magnetic connection to the Earth: spacecraft 1 is followed by spacecraft 3 , then by spacecraft 2 and finally by spacecraft 4 . In agreement with this figure (Fig. 38), spacecraft 1 is the first arriving in the polar cap followed by spacecraft 3 and spacecraft 4 .

These results fit the general pattern of ionospheric ion acceleration and plasma sheet ion precipitation near the polar boundary of the auroral oval reported from FAST, POLAR and INTERBALL measurements. However, Cluster will be able to determine the characterisric times of the ion acceleration/precipitation processes for the first time.

\subsection{RPA measurements}

One example of data obtained in the RPA mode (14 February 2001) is shown in Fig. 40 with CODIF on spacecraft 3. At the top of the figure, ion fluxes measured by HIA in 4 directions, from 04:00 to 12:00 UT, are shown; at the bottom, RPA measurements between 0 and $25 \mathrm{eV} / \mathrm{e}$ for the 4 ion species and the ion density deduced from HIA are shown. Low energy $\mathrm{H}^{+}$ions are measured primarily between 04:30 and $07.00 \mathrm{UT}$, then later, at the entrance in the magnetosheath when RPA measures the low energy part of the $\mathrm{H}^{+}$ions. Note, however, that the counting rate scales are very different for HIA and for RPA.

6.10 Influence of ASPOC on the low energy ion measurements

The ASPOC instrument (Riedler et al., 1997) is designed to emit indium ions from a source to control the spacecraft potential. Effectively, the CIS instrument has seen the positive effect of ASPOC for ion measurements. Figure 41 presents the CIS CODIF data obtained on 4 February 2001 for the 3 spacecraft Rumba, Samba and Tango: on Rumba and Samba, CODIF is operating in the RPA mode from 14:36 UT to 15:36 UT, while on Tango, CODIF is operating in the RPA mode from 14:41 UT to 15:41 UT. Then, the CIS instruments are operated in a magnetospheric mode. Although during magnetospheric modes the CODIF energy range goes up to about $38 \mathrm{keV} / \mathrm{e}$, the energy scale is limited on this plot up to $300 \mathrm{eV}$, just to focus on the effects of the low energy population. On spacecraft 1, ASPOC is not operating; on spacecraft 3, ASPOC stops emission at 15:19:57 UT and on spacecraft 4, ASPOC stops the emission at 17:40:57 UT. The switch-off of the ASPOC beam is clearly observable on the CIS data. On spacecraft 3, CIS stops observing low energy $\mathrm{H}^{+}$and $\mathrm{He}^{+}$after 15:19:57. On spacecraft 4, CIS stops observing $\mathrm{H}^{+}$and $\mathrm{He}^{+}$after 17:40:57. The effect is particularly clear on the $\mathrm{He}^{+}$population $(20$ to $70 \mathrm{eV}$ ), observed on spacecraft 4 from about 16:40 UT, and never observed on the other two spacecraft, on which the ASPOC beam was off during that interval. $\mathrm{He}^{+}$is a minority ion species of ionospheric origin; it cannot be detected at these low energies without the spacecraft potential neutralisation by ASPOC. The effect is also small and very clear on spacecraft 4 at the same time with the decrease of $\mathrm{H}^{+}$ion fluxes below $100 \mathrm{eV}$, when ASPOC is turned off. By lowering the spacecraft potential, ASPOC has a positive effect for the detection of low energy ions that are not normally detected.

\section{Conclusion}

The general characteristics of the two CIS instruments, including scientific performances, weight and raw power are summarised in Table 1. Note that the entrance of each sensor is placed about $10 \mathrm{~cm}$ outside the spacecraft platform in order to have an unobstructed field of view and to minimise the effect of the spacecraft potential on the trajectories of the low-energy particles. The two planes of view of CODIF and HIA, mounted on opposite sides of the spacecraft, are parallel and tangential to the spacecraft body. The field of view of the two sensors is $15^{\circ} \times 360^{\circ}$. 


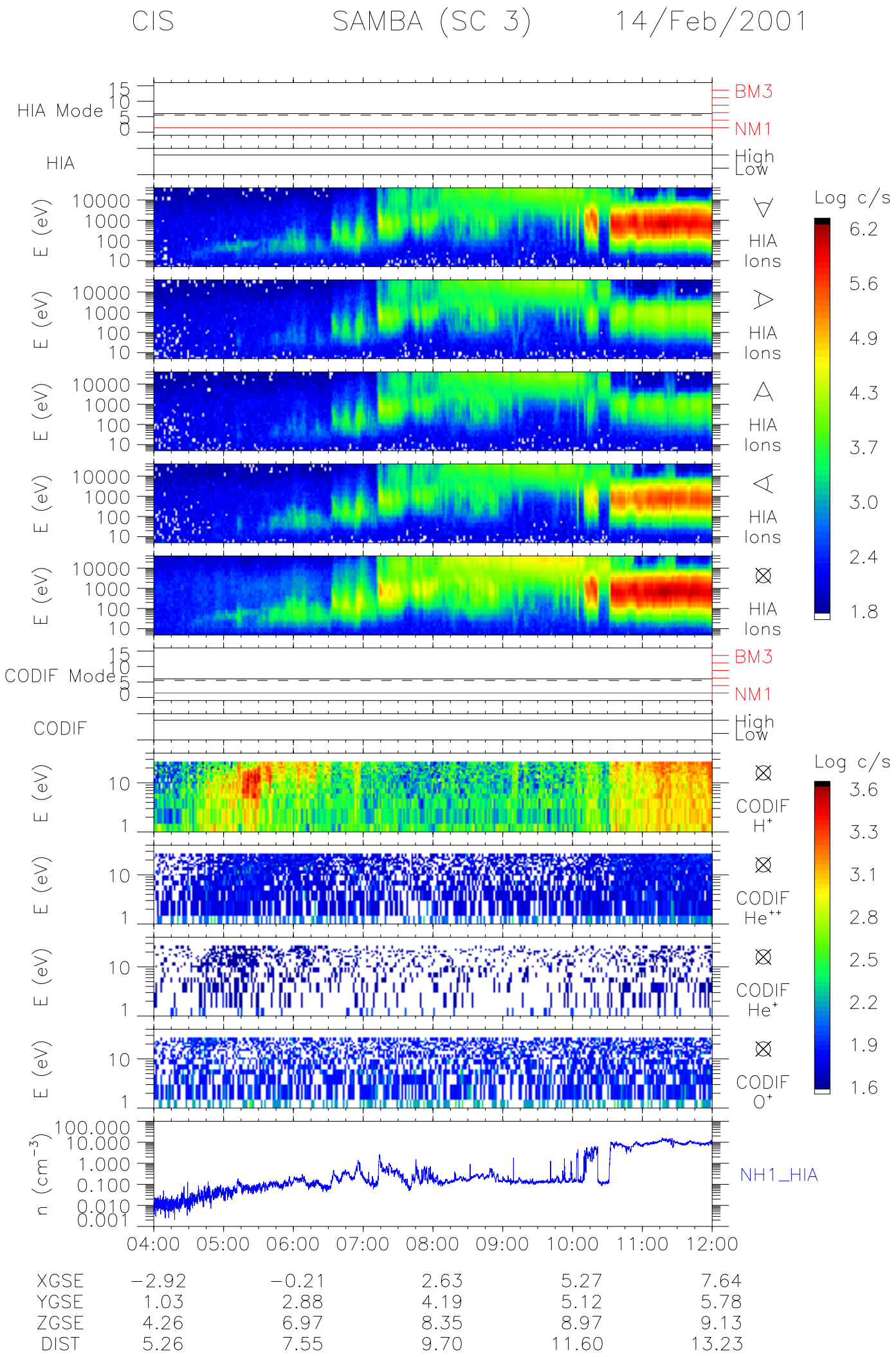

Fig. 40. Example of RPA CODIF measurements with spacecraft 3, between $\sim 0$ and $25 \mathrm{eV}$, on 14 February 2001, between 04:00 and 12:00 UT. From top to bottom: HIA telemetry modes and sensitivities, energy-time spectrograms measured in the sunward, dusk, antisunward, dawnward looking directions and energy-time spectrogram integrated over $4 \Pi$; CODIF telemetry modes and sensitivities, $\mathrm{H}^{+}$, $\mathrm{He}^{++}, \mathrm{He}^{+}$, and $\mathrm{O}^{+}$energy-time spectrogram integrated over $4 \Pi$, between 1 and $25 \mathrm{eV}$, HIA and CODIF densities. 


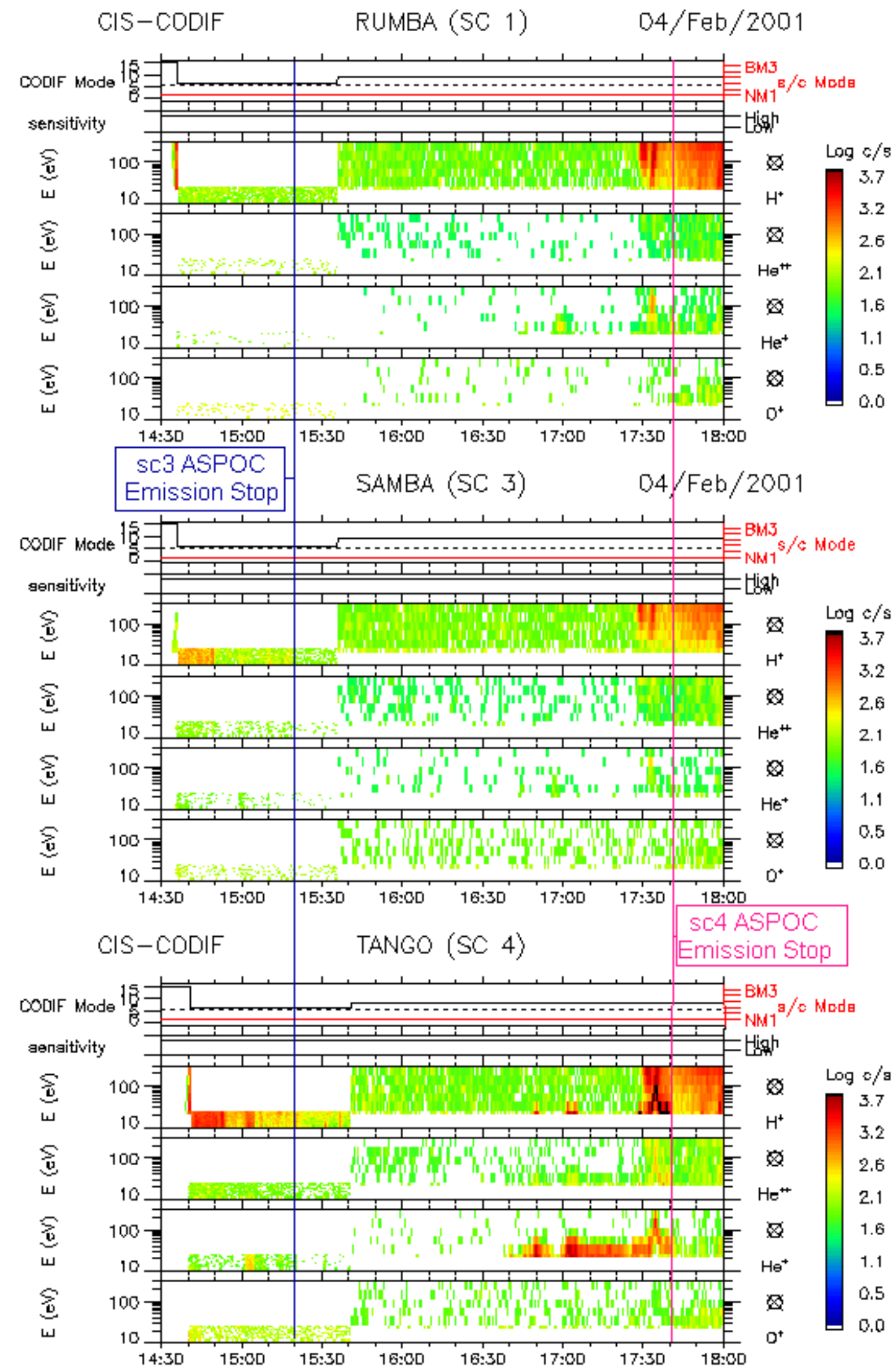

Fig. 41. Effect on ASPOC for low energy ion measurements, on 4 February 2001: CODIF energy-time spectrograms, integrated over 4 П, for $\mathrm{H}^{+}, \mathrm{He}^{++}, \mathrm{He}^{+}$, and $\mathrm{O}^{+}$ions on spacecraft 1,3, and 4 are shown. On spacecraft 1 ASPOC does not work; on spacecraft 3 ASPOC stops at 15:20 UT; on spacecraft 4, ASPOC stops at 17:41 UT. 
In summary, by their unique features, the CIS instruments provide fast measurements of the major plasma ion species with greatly improved accuracy and resolution. The inherent flexibility of the instrument control allows for a permanent optimisation of the scientific operation according to the various situations encountered along the Cluster orbits. The extensive onboard data processing and compression not only improve the time resolution of the measurements and significantly reduce data ground-processing costs, but also make the plasma fundamental parameters available quickly and directly in a usable form to the scientific community.

The first results presented in this paper show that even with only 3 spacecraft, CIS is able to have, in a near future, a major contribution to the knowledge of the magnetosphere and its interaction with the solar wind.

Acknowledgements. Cluster 2 decision was only possible thanks to Dr. Roger BONNET, Science Director at ESA. We thank also ESA and DORNIER Project Teams, Starsem and Soyouz/Fregat Teams, ESOC and JSOC. The Cluster 2 CIS instrument was supported by ESA and by many institutions: CESR had the overall responsibility of the instruments, has fabricated the CODIF analyser, DPU CODIF boards, HIA detector, part of HIA electronics and HIA DPU, has participated to the integration, environmental and spacecraft tests and CODIF calibrations, has done the HIA calibrations and data analysis software. UNH has built the CODIF detector, the TOF digital electronics and has participated to the CODIF calibrations. MPE Garching has built the CODIF acceleration high voltage and the CODIF MCP high voltage, the time of flight electronics and the TAC converter. IFSI Roma has built the HIA analyser, part of the HIA electronics, and the HIA flight software. MPAE Lindau has built the CODIF analyser high voltage and the HIA analyser and MCP high voltages. IRF Kiruna has done the lay out of the CODIF DPU boards. SSL at UC Berkeley has done the DPU flight software and the GSE software. UW has done the CODIF RPA calibrations and the CODIF calibrations were done at the University of Bern. At CESR financial support came from CNES grant. The contribution of MPE Garching was supported by ESA under contract 1501073-2400 and by DLR (Deutsches Zentrum für Luft und Raumfahrt) under contracts 50 OC 8906 and 50 OC 0102. The high voltage supply of CIS-2 was provided by MPAE Lindau with the support of the Max-Planck-Gesellschaft zur Förderung der Wissenschaften and DARA. For IFSI, CIS experiment was supported by Italian Space Agency (ASI). The work at the University of Washington, UNH, LPARL, and UC Berkeley was supported by NASA contract. The Swedish participation was funded by the Swedish National Space Board and the Swiss participation by the Swiss National Foundation and the State of Bern. The authors thank Mrs. P. Décréau for the Whisper data.

Topical Editor G. C. Chanteur thanks D. G. Mitchell and another referee for their help in evaluating this paper.

\section{References}

Andre, M. and Yau, A. W.: Theories and observations of ion energization and outflow in the high latitude magnetosphere, Space Sci. Rev., 80, 24, 1997.

Bosqued, J. M., Phan, T. D., Dandouras, I., et al.: CLUSTER Observation of the high-latitude magnetopause and cusp: First Re- sults from the CIS Ion Instruments, Ann. Geophysicae, this issue, 2001.

Burgess, D.: Cyclic behaviour at quasi-parallel collisionless shocks, Geophys. Res. Lett. 16, 345, 1989.

Carlson, C. W., Curtis, D. W., Paschmann, G., et al.: An instrument for rapidly measuring plasma distribution functions with high resolution, Adv. Space Res. 2, 7, 67, 1982.

Chappell, C. R.: The terrestrial plasma source: a new perspective in solar-terrestrial processes from Dynamics Explorer, Rev. Geophys., 26, 4757, 1988.

Cowley S. W. H.: The causes of convection in the earth's magnetosphere - A review of developments during the IMS, Rev. Geophys. Space Phys., 20, 531, 1982.

Dungey, J. W.: Interplanetary magnetic field and the auroral zones, Phys. Rev. Lett., 6, 47, 1961.

Escoubet, P. C., Schmidt, R., and Goldstein, M. L.: Cluster Science and Mission Overview, Space Sci. Rev., 79, 11, 1997.

Giacalone, J., Schwartz, S. J., and Burgess, D.: Artificial spacecraft in hybrid simulations of the quasi-parallel Earth's bow shock: Analysis of time series versus spatial profiles and a separation strategy for Cluster, Ann. Geophysicae, 12, 591, 1994.

Gloeckler, G., Geiss, J., Balsiger, H., et al.: Detection of interstellar pick-up hydrogen in the Solar System, Science, 261, 70, 1993.

Fujimoto, M., Nishida, A., Mukai, T., Saito, Y., Yamamoto, T., and Kokubun, S.: Plasma sheet entry from the flanks of the nearEarth magnetotail: Geotail observations in the dawnside LLBL and the plasma sheet, J. Geomag. Geoelectr., 48, 711, 1996.

Fujimoto, M., Terasawa, T., Mukai, T., Saito, Y., Yamamoto, T., and Kokubun, S.: Plasma entry from the flanks of the near-Earth magnetotail: Geotail observations, J. Geophys. Res., 103, 4391, 1998.

Fuselier, S. A., Anderson, B. J., and Onsager, T. G.: Electron and ion signatures of field line topology at the low-shear magnetopause, J. Geophys. Res., 102, 4847, 1997.

Fuselier, S. A., Elphic, R. C., and Gosling, J. T.: Composition measurements in the dusk flank magnetosphere, J. Geophys. Res. 104, 4515, 1999.

Kistler, L. M.: Cluster CODIF Calibration report, Part I, II and III, 2000.

Kondo, T., Whalen, B. A., Yau, A. W., and Peterson, W. K.: Statistical analysis of upflowing ion beam and conic distributions at DE-1 altitudes, J. Geophys. Res., 95, 12 091, 1990.

Lin, R. P., Anderson, K. A., Ashford, S., et al.: A three-dimensional plasma and energetic particle investigation for the wind spacecraft, Space Sci. Rev. 71, 125, 1995.

Lockwood, M., and Smith, M. F.: The variation of reconnection rate at the dayside magnetopause and cusp ion precipitation, J. Geophys. Res., 97, 14 841, 1992.

McCarthy, M.: Cluster 2 CIS RPA Calibration report, 14 March 2000.

Martz C.: Spectrométrie Ionique dans la Magnétosphère et le Vent Solaire. Simulation et Précision des Mesures Coordonnées au Moyen des 4 Satellites de la Mission Cluster, Thesis, Paul Sabatier Toulouse University, 1993.

Möbius, E., Hovestadt, D., Klecker, B., et al.: The time-of-flight spectrometer SULEICA for ions of the energy range 5-270 keV/charge on AMPTE IRM, IEEE Trans. Geosc. Remote Sens. GE-23, 274, 1985.

Möbius, E., Hovestadt, D., Klecker, B., et al.: Direct Observation of $\mathrm{He}^{+}$Pick-up Ions of Interstellar Origin in the Solar Wind, Nature 318, 426, 1985.

Möbius, E., Klecker, B., Hovestadt, D., et al.: Interaction of inter- 
stellar pick-up ions with the solar wind, Astrophys. Space Sci. 144, 487, 1988.

Paschmann, G., Loidl, H., Obermayer, P., et al.: The plasma instrument for AMPTE IRM, IEEE Trans. Geosc. Remote Sens. GE-23, 262, 1985.

Paschmann, G., Baumjohann, W., Sckopke, N., et al.: The magnetopause for large magnetic shear: AMPTE/IRM Observations, J. Geophys. Res. 91, 11 099, 1986.

Phan, T. D., Lin, R. P., Fuselier, S. A., and Fujimoto, M.: Wind observations of mixed magnetosheath-plasma sheet ions deep inside the magnetosphere, J. Geophys. Res., 105, 5497, 2000.

Quest, K. B.: Theory and simultion of collisionless parallel shocks, J. Geophys. Res. 93, 9649, 1987.

Rème, H., Cottin, F., Cros, A., et al.: The Cluster Ion Spectrometry (CIS) Experiment, Space Sciences Review 79, 303-350, 1997.

Rème, H., Bosqued, J.-M., Sauvaud, J.-A., et al.: The Giotto Electron Plasma Experiment, J. Phys. E: Sci. Instrum. 20, 721-731, 1987.

Riedler, W., Torkar, K., Rudenauer, F., et al.: Active Spacecraft Potential Control, Space Sciences Review 79, 271-302, 1997.

Ritter, H.: Sekundärelektronenemission von Kohlenstoffolien beim Durchgang von Ionen im Energiebereich von 40 bis $500 \mathrm{keV}$, MPE Report 190, 1985.

Sauvaud, J.-A., Popescu, D., Delcourt, D. C., et al.: Sporadic plasma sheet ion injections into the high altitude auroral bulge: satellite observations, J. Geophys. Res., 104, 28 565, 1999.

Scholer, M. and Terasawa, T.: Ion Reflection and Dissipation at Quasi-parallel Collisionless Shocks, Geophys. Res. Lett. 17, 119, 1990.

Scholer, M.: Interaction of upstream diffuse ions with the solar wind, Adv. Space Sci., 1997.
Sckopke, N., Paschmann, G., Haerendel, G., Sonnerup, B. U. O., Bame, S. J., Forbes, T. G., Hones, E. W., and Russell, Jr., C. T.: Structure of the low-latitude boundary later, J. Geophys. Res., 86, 2099, 1981.

Sckopke N., Paschmann, G, Brinca, A. L., et al.: Ion Thermalization in Quasi-perpendicular Shocks Involving Reflected Ions, J. Geophys. Res. 95, 6337, 1990.

Sergeev, V. A., Sauvaud, J.-A., Popescu, D., et al.: Plasma sheet ion injections into the auroral bulge: correlative study of spacecraft and ground observations, J. Geophys. Res., 105, 18 465, 2000.

Smith, M. F. and Lockwood, M.: Earth's magnetospheric cusps, Rev. Geophys., 34, 233, 1996.

Stasiewicz, K.: New Methods and Techniques in Visualization and Mapping of Magnetospheric Boundaries, Physical Signatures of Magnetospheric Boundary Layer Processes, (Eds) Holtet, J. A. and Egeland, A., Kluwer Academic Publishers, 433-447, 1994.

Stasiewicz, K.: Orbit Visualization Tool-2 (OVT) for CLUSTER, http://ovt.irfu.se, 2001.

Terasawa, T., Fujimoto, M., Mukai, T., et al.: Solar wind control of density and temperature in the near-Earth plasma sheet: WIND/GEOTAIL collaboration, Geophys. Res. Lett., 24, 935, 1997.

Trattner, K. J., Möbius, E., Scholer, M., et al.: Statistical Analysis of Diffuse Ion Events Upstream at the Earth's Bow Shock, J. Geophys. Res. 99, 13 389, 1994.

Williams, D. J., Mitchell, D. G., Eastman, T. E., and Frank, L. A.: Energetic particle observations in the low-latitude boundary layer, J. Geophys. Res., 90, 5097, 1985.

Young D. T., Bame, S. J., Thomsen, M. F., et al.: 2 П-radian fieldof-view toroidal electrostatic analyzer, Rev. Sci. Instr., 59, 743, 1988. 